\title{
MR angiography of the lower extremities
}

\author{
Citation for published version (APA):
}

Ho, K. Y. J. A. M. (1999). MR angiography of the lower extremities. [Doctoral Thesis, Maastricht University]. Universiteit Maastricht. https://doi.org/10.26481/dis.19991216kh

Document status and date:

Published: 01/01/1999

DOI:

10.26481/dis.19991216kh

Document Version:

Publisher's PDF, also known as Version of record

\section{Please check the document version of this publication:}

- A submitted manuscript is the version of the article upon submission and before peer-review. There can be important differences between the submitted version and the official published version of record.

People interested in the research are advised to contact the author for the final version of the publication, or visit the DOI to the publisher's website.

- The final author version and the galley proof are versions of the publication after peer review.

- The final published version features the final layout of the paper including the volume, issue and page numbers.

Link to publication

\footnotetext{
General rights rights.

- You may freely distribute the URL identifying the publication in the public portal. please follow below link for the End User Agreement:

www.umlib.nl/taverne-license

Take down policy

If you believe that this document breaches copyright please contact us at:

repository@maastrichtuniversity.nl

providing details and we will investigate your claim.
}

Copyright and moral rights for the publications made accessible in the public portal are retained by the authors and/or other copyright owners and it is a condition of accessing publications that users recognise and abide by the legal requirements associated with these

- Users may download and print one copy of any publication from the public portal for the purpose of private study or research.

- You may not further distribute the material or use it for any profit-making activity or commercial gain

If the publication is distributed under the terms of Article $25 \mathrm{fa}$ of the Dutch Copyright Act, indicated by the "Taverne" license above, 


\section{MR Angiography of the Lower Extremities}





\title{
MR Angiography of the Lower Extremities
}

\author{
PROEFSCHRIFT \\ ter verkrijging van de graad van doctor \\ aan de Universiteit Maastricht \\ op gezag van de Rector Magnificus, \\ Prof. dr. A.C. Nieuwenhuijzen Kruseman, \\ volgens het besluit van het College van Decanen, \\ in het openbaar te verdedigen \\ op donderdag 16 december 1999 om 14.00 uur
}

Door

K.Y.J.A.M. Ho 


\section{Promotores:}

Prof. dr. J.M.A. van Engelshoven

Prof. dr. P.J.E.H.M. Kitslaar

\section{Beoordelingscommissie:}

Prof. dr. A.P.G. Hoeks (voorzitter)

Prof. dr. F.W.H.M. Bär

Prof. dr. J.H. van Bockel

Prof. dr. P.W. de Leeuw

Prof. dr. W.P.Th.M. Mali

The publication of this thesis was financially supported by: Schering AG And: 


\title{
MR Angiografie \\ van de Onderste Extremiteiten
}

\author{
( met een samenvatting in het Nederlands)
}



献给我最敞的色色 



\section{Contents}

$\begin{array}{lll}\text { Chapter } 1 \text { Introduction } & 1\end{array}$

Chapter 2 Peripheral MR Angiography 9

Chapter $3 \quad$ MR Angiography of the Iliac and Upper Femoral 27

Arteries using Four Different Inflow Techniques

Chapter $4 \quad$ Peripheral Vascular Tree Stenoses: Detection with 47

Subtracted and Nonsubtracted MR angiography

Chapter $5 \quad$ Peripheral Vascular Tree Stenoses: Evaluation with $\quad 69$

Moving-Bed Infusion-tracking MR Angiography

Chapter $6 \quad$ Analysis of Image Interpretability in Contrast-enhanced 93

Peripheral MR Angiography using the MoBI-track technique

Chapter $7 \quad$ Peripheral Vascular Tree Stenoses: Evaluation with

Bloodpool-enhanced and Gd-DTPA-enhanced MR

Angiography

Chapter $8 \quad$ MR Angiography: Runoff Vessels

Chapter $9 \quad$ General Discussion

$\begin{array}{ll}\text { Summary } & 149\end{array}$

$\begin{array}{ll}\text { Samenvatting } & 155\end{array}$

$\begin{array}{ll}\text { Curriculum Vitae } & 161\end{array}$

$\begin{array}{ll}\text { Dankwoord } & 167\end{array}$

Starting from page 1 you can flip through the pages. If you focus on the right lower corner, you will be able to appreciate the three-dimensional nature of a peripheral contrast-enhanced MR angiographic dataset of the pelvic arteries. 



\title{
Chapter 1
}

\author{
Introduction
}





\section{Introduction}

Peripheral vascular obstructive disease (PVOD) is a common disorder in western society which is caused by atherosclerotic disease in $80-90 \%$ of cases [1]. The incidence as shown by the results of the Transition project was 2.8 newly discovered cases of patients with PVOD per 1000 patients per year [2]. However, as PVOD is a progressive disease, many more patients consult a doctor each year as the prevalence of registered symptomatic PVOD in Dutch general practice is $4.7 \%$ [2]. This number is likely to increase with the expected increased lifespan of people, as PVOD is positively correlated with age (prevalence in people older than 75 years of age $>20 \%$ [2]). Fortunately, improved surgical and percutaneous transluminal angioplasty techniques for the treatment of PVOD are now available. However, accurate diagnosis by detection, grading and characterization of atherosclerotic lesions are essential prerequisites for a positive outcome of treatment.

\section{Diagnostic Workup in Peripheral Vascular Obstructive Disease}

In the Netherlands, the diagnostic workup of a patient with suspected PVOD consists of a two-step approach. The first step consists of history taking, and determination of the ankle-brachial index which can be supplemented with a walking test if the diagnosis concerning PVOD is not certain. The second step in patients with suspected PVOD and who are eligible for arterial intervention, consists of Duplex ultrasonography or diagnostic X-ray angiography. Being a noninvasive test with relatively high sensitivity and specificity figures for detecting and grading hemodynamically significant stenoses ( $250 \%$ lumen reduction) of $80-86 \%$ and $>95 \%$, respectively, Duplex ultrasonography is the preferred procedure in the Netherlands [1]. However, Duplex ultrasonography has disadvantages. In $10 \%$ of patients, the examination is unsuccessful because of various errors (bowel gas, severe calcification of the arterial wall), the examination itself is very operator dependent, time consuming and is not available in all hospitals. Even more so, in multi-stage disease (involvement of multiple arterial segments with multiple stenoses), Duplex ultrasonography becomes less reliable and in the crural arteries, Duplex ultrasonography has not proven its efficacy yet [1].

Diagnostic X-ray angiography, being the standard of reference for a long time, is not hampered by these limitations. The information acquired with this technique has very high inplane resolution and percutaneous transluminal angioplasty aimed at treating the diseased vessel segment, can be performed in the same session. However, being an invasive test with the use of nephrotoxic contrast agents with a small but definite risk of moderate to severe side-effects $(0.2 \%$ for developing acute renal failure, and an overall adverse event rate of $9.5 \%$ [3]), in the Netherlands X-ray angiography is reserved for those patients who are to undergo angioplasty [1]. 


\section{MR Angiography as an Alternative}

A non- or minimally-invasive alternative to both Duplex ultrasonography and diagnostic X-ray angiography, capable of acquiring both hemodynamic as well as morphologic information of the peripheral vasculature, is Magnetic Resonance angiography (MR angiography). This outpatient imaging modality uses strong magnetic fields and radiofrequent pulses to acquire data of the human body without the need for ionizing radiation. Even more so, if contrast agents (gadolinium-chelates) are used to improve vessel-to-background contrast these intravenously injected compounds (maximum volume $30-40-\mathrm{mL}$ ) have a far better safety profile than iodinated contrast agents (overall adverse event rates for contrast-enhanced MRI using gadolinium-chelates are in the order of $0.003-0.01 \%$ in two large series comprising a total of 34,439 patients $[4,5]$ ) and are nonnephrotoxic. Furthermore, the examination is much less operator dependent as compared to Duplex ultrasonography, and acquires high resolution three-dimensional data in a single breathhold, that during postprocessing can be displayed in any desired obliquity and with several display methods (maximum intensity projections, exoscopic and endoscopic viewing modes [fig. 1]).

A

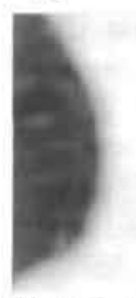

B

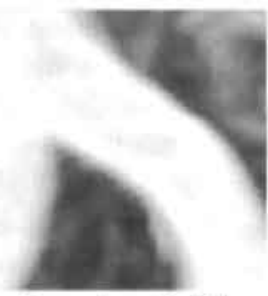

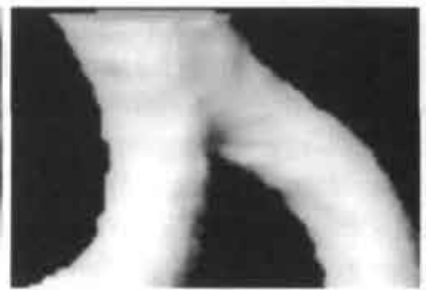

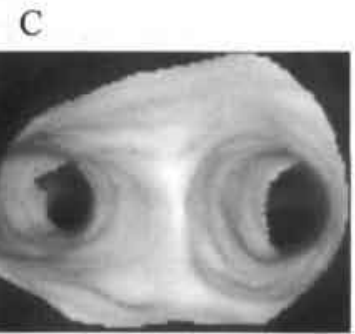

Figure 1. Examples of different display methods. A: Maximum intensity projection of the aortic bifurcation. B: Shaded surface display (exoscopic view) of the aortic bifurcation. C: Endoscopic view (cranio-caudal view) of the aortic bifurcation. (Three-dimensional views of these three display methods can be seen on the Image-CD\}.

Although there are contra-indications for some patients (claustrophobia, some pacemakers), it is our experience that most patients $(>95 \%)$ can be imaged successfully.

\section{The Objectives of this Thesis are:}

1. To evaluate the use of Magnetic Resonance Imaging for depiction of the peripheral vascular tree.

2. To assess the accuracy of MR angiography in detecting and grading hemodynamically significant stenoses ( $250 \%$ lumen reduction) in the peripheral vascular tree with different MR angiographic techniques. 
3. To develop a contrast-enhanced MR angiographic technique for imaging the entire peripheral vascular tree (aorta down to the feet) in a total patient handling time of less than 30 minutes.

4. To evaluate image interpretability of contrast-enhanced MR angiography of the entire peripheral vascular tree in a large patient population (131 patients).

5. To evaluate the feasibility of a new contrast material (bloodpool agent), enabling the acquisition of ultrahigh resolution data.

\section{Outline of this Thesis:}

In chapter 2, working mechanisms and pitfalls of time-of-flight MR angiography and contrast-enhanced MR angiography for imaging the peripheral arteries are explained and information about usefulness and validity of both techniques as noted in recent literature is given.

In chapter 3, a prospective comparison was used to demonstrate which time-offlight MR angiographic technique produces best results in patients with intermittent claudication for imaging the pelvic arteries. For this purpose, image quality and accuracy concerning detection and grading of stenoses of four different time-of-flight MR angiographic techniques (two different pulse sequences both with and without systolic synchronization) were compared with diagnostic X-ray angiography as the standard of reference.

In chapter 4 , the results of a comparison between three-dimensional gadoliniumenhanced MR angiography (subtracted and nonsubtracted) and two-dimensional systolic synchronized time-of-flight MR angiography are described, using X-ray angiography as the standard of reference.

In chapter 5, a peripheral gadolinium-enhanced MR angiographic technique imaging the entire peripheral vascular tree (aorta down to the feet) using only one single bolus of contrast material in four minutes (Moving-Bed Infusion-tracking technique [MoBI-track]) in both volunteers and patients is described. Furthermore, using the MoBI-track technique, the results in patients compared with X-ray angiography as the standard of reference are described.

In chapter 6 , results are described concerning image interpretability of peripheral contrast-enhanced MR Angiography using the MoBI-track technique and techniqueand patient- related factors which may influence image interpretability are defined. Furthermore, suggestions are given for future improvements.

In chapter 7, a study is described that investigated the feasibility of bloodpool-

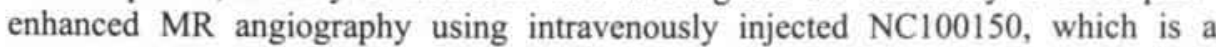
starch-coated iron-oxide particle bloodpool agent for imaging the peripheral arteries in 10 patients. The results of bloodpool-enhanced MR angiography were compared with gadolinium-enhanced MR angiography, using diagnostic X-ray angiography as the standard of reference.

In chapter 8 , working mechanisms of three bolus imaging strategies for evaluation of the entire peripheral vascular tree are explained. The advantages and disadvantages of each technique are described and results as noted in recent literature are provided. 
Chapter 9 discusses the impact this thesis has had on the current status of MR angiography, provides an outlook on the clinical impact of the MoBI-track technique and gives the conclusions of this thesis. 


\section{References}

1. Kitslaar P.J.E.H.M., Hartbulletin, themanummer Consensus arteriële claudicatio intermittens. $\mathrm{Nr}$ 6; dec 1997: 174-180.

2. Banga J.D., Stoffers H.E.J.H., Berengoltz-Zlochin S.. Kliniek, epidemiologie en prognose. Hartbulletin $\mathrm{Nr}$ 6; dec 1997: 185-189.

3. Waugh J.R., Sacharias N. (1992) Arteriographic complications in the DSA era. Radiology 182: 243-246.

4. Murphy K.J., Brunberg J.A., Cohan R.H. (1996) Adverse reactions to gadolinium contrast media: a review of 36 cases. Am J Roentgenol 167: 847-849.

5. Niendorf H.P., Haustein J., Cornelius I., Alhassan A., Clauss W. (1991) Safety of gadolinium-DTPA: extended clinical experience. Magn Reson Med 22: 222-228. 
Chapter 1 


\section{Chapter 2}

\section{Peripheral MR angiography}

This article is accepted for publication as an invited article in European Radiology

Authors: Ho K.Y.J.A.M., Leiner T., de Haan M.W., van Engelshoven J.M.A. 



\section{Abstract}

Atherosclerotic disease of the lower extremities is a common disorder in western society. Its debilitating nature calls for accurate diagnosis and treatment. The gold standard for diagnosing this disease by depiction of vessel morphology is diagnostic $\mathrm{X}$-ray angiography (either conventional or digital subtraction angiography). However, the invasive nature of this technique and the possible harmful effects of iodinated contrast agents have led to the idea that noninvasive MR angiography might be a good alternative for acquiring information about vessel morphology.

Most extensively studied was time-of-flight MR angiography. Although first results with this technique were encouraging, it is now apparent that time-of-flight MR angiography is hampered by the virtue of which it exists.

However, with the introduction of minimally invasive contrast-enhanced MR angiography, using gadolinium-chelates to reduce T1 of blood, image quality has improved tremendously. Even more so, using contrast-enhanced MR angiography, high resolution three-dimensional data of the entire peripheral vascular tree can be obtained within several minutes, which might make MR angiography a true competitor of X-ray angiography as a diagnostic tool in the clinical workup of a patient with complaints of peripheral vascular obstructive disease.

The purpose of this article is to explain working mechanisms and usefulness of both time-of-flight and contrast-enhanced MR angiography. 
Chapter 2 


\section{Introduction}

MR angiography has received a lot of attention over the past decade since its first introduction in 1985 by Wedeen et al [1,2]. Many different techniques have been used, but most are hampered by artifacts or time limitations. Most commonly used techniques for imaging the peripheral arteries are time-of-flight (TOF) MR angiography and contrast-enhanced (CE) MR angiography using gadoliniumchelates. The latter of these two techniques seems to be the most promising, and has already proven its potential for imaging the entire peripheral vascular tree (complete outflow study) in several minutes [3-5].

The purpose of this article is to explain working mechanisms and pitfalls of TOF MR angiography and CE MR angiography for imaging the peripheral arteries and to give information about usefulness and validity of both techniques as noted in recent literature.

\section{Time-of-flight MR Angiography}

TOF MR angiography has been used for imaging the peripheral arteries in many studies [6-13]. Most of these were two-dimensional (2D) methods, applied with variable success. 2D-TOF MR angiography is a relatively simple technique based on the inflow of fresh unsaturated blood in a saturated slice. Saturation of the (background-)tissue in the slice is achieved by submitting it to radiofrequent (RF) pulses with a repetition time (TR) much shorter than tissue Tl values, thereby decreasing its longitudinal magnetization vector. Since inflowing (unsaturated) blood still has a large longitudinal magnetization vector, it will be seen in the imaged slice as an area of high signal intensity.

The purpose of any MR angiographic technique is to obtain adequate diagnostic information by maximizing vessel-to-background contrast. In 2D-TOF MR angiography, vessel signal intensities can be maximized by keeping the transit time of blood in the imaged slice as short as possible to prevent saturation of blood by repetitive RF pulses. Maximum signal intensity of blood is achieved if it receives only one RF pulse while flowing through the imaged slice. Further pulses will only saturate the blood as it does background tissue. Therefore, in order to minimize intraslice transit time and maximize vessel-to-background contrast, imaged slices in TOF MR angiography are planned perpendicular to the vessel of interest, which in the case of the peripheral arteries means an axial orientation. Another method of decreasing intraslice transit time is to image the slice during maximum flow of blood which is in the systolic window of the heart cycle. This technique is known as systolic cardiac synchronization (either by triggering or gating).

Since any unsaturated spins entering an imaged slice create a time-of-flight effect, veins too can give rise to increased signal intensities. To suppress this signal a tracking presaturation slab caudal to the imaged slice is used. This presaturation slab subjects venous blood to multiple RF pulses, resulting in saturation. Therefore, when it flows into the imaged slice, it already is saturated and will not appear in the imaged slice. 


\section{Imaging Parameters}

As with all MR angiographic techniques, TOF MR angiography is subjected to a trade-off between image resolution, acquisition time and vessel-to-background contrast. Table 1 tabulates the impact of different scanparameters on: returned signal from a voxel, vessel-to-background contrast, image resolution and acquisition time.

Table 1. Complex Effects on Voxel Signal, Vessel-to-Background Contrast, Resolution and Acquisition Time when increasing Different Time-of-flight sequence parameters.

$\begin{array}{lllll}\text { Increasing parameters } & \text { Voxel Signal } & \text { VB contrast } & \text { Resolution } & \text { Acquisition Time } \\ \text { FOV } & 1 & \text { tor } 1 & 1 & - \\ \text { Matrix } & 1 & \text { tor } 1 & 1 & 1 \\ \text { Slice Thickness } & 1 & \text { tor } 1 & 1 & - \\ \text { rFOV } & - & 1 & - & 1 \\ \text { TR } & 1 & \text { or } 1 & - & 1 \\ \text { TE } & \text { tor } 1 & \text { or } 1 & - & - \text { or } 1 \\ \text { FA } & 1 & \text { or } 1 & - & \text { - or } 1\end{array}$

VB contrast $=$ vessel-to-background contrast, $\mathrm{FOV}=$ field of view, $\mathrm{rFOV}=$ rectangular field of view, $\mathrm{TR}=$ repetition time, TE $=$ time-to-echo, $F A=$ flip angle, $t=$ increasing effect,$!=$ decreasing effect, - no effect.

Field of view (FOV) and matrix size both result in a changed voxel size in frequency and phase encoding directions. Increasing the voxel size can increase voxel signal and therefore increase vessel-to-background contrast. However, if voxels become larger than the vessels of interest, partial volume effects can decrease vesselto-background contrast. Changing slice thickness can have more or less the same effect as changing FOV and matrix size. However, if slice thickness is increased, it can become too thick and intraslice transit time of blood is prolonged, resulting in inplane saturation (see "acquisition and interpretation pitfalls").

Using a rectangular FOV ( $\mathrm{rFOV}$ ) decreases acquisition time but also decreases signal-to-noise ratios (SNR). Therefore this has a negative effect on vessel-tobackground contrast.

Increasing TR values increases signal from a voxel. However, this is true for both voxels containing blood and background tissue. Therefore increasing TR can have a negative effect on vessel-to-background contrast. On the other hand, decreasing TR values can give good background saturation if blood does not receives too many saturating RF pulses while flowing through the imaged slice. Especially in diastole when there is slow flow, blood too can become saturated, resulting in inplane saturation.

Increasing time-to-echo (TE) usually also increases TR. Although a shorter TE decreases flow voids induced by poststenotic turbulence, at a TE of $6.9-\mathrm{msec}$ (on a 1.5 Tesla MR system), water and fat are out of phase and therefore, vessel-tobackground contrast in background tissue containing fat can be increased with the longer TE value [14].

Increasing the flip angle (FA), increases the saturation effect given by one RF pulse. Therefore larger angles give better background suppression. However, if blood receives more than one RF pulse while in the imaged slice, an increased FA also results in faster saturation of blood, decreasing vessel-to-background contrast. 
Other methods of increasing vessel-to-background contrast can be implemented in the sequence. The most effective method is the use of systolic cardiac synchronization [9]. This method not only ensures the shortest intraslice transit time (imaging with maximum flow velocities), but also prevents artifacts caused by vessel pulsatility and variations in flow velocities and directions across the cardiac cycle. However, if vessel pulsatility and flow variations are reduced, particularly distal to severe stenoses, systolic cardiac synchronization can be of little added value. Disadvantages of systolic cardiac synchronization are increased acquisition time and increased length of post-stenotic flow voids.

\section{Acquisition and Interpretation Pitfalls}

Two major types of artifacts can be distinguished in TOF MR angiography: flow voids and ghosting artifacts. The latter, being caused by vessel pulsatility and large variations in flow velocity during the cardiac cycle, can be prevented by using (systolic) cardiac synchronization. Figure 1 clearly demonstrates the increased image quality achieved by using systolic cardiac synchronization.

A

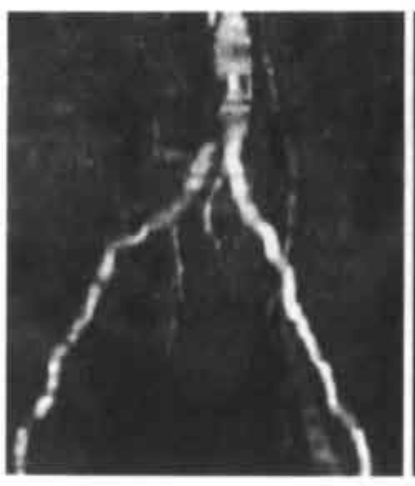

B

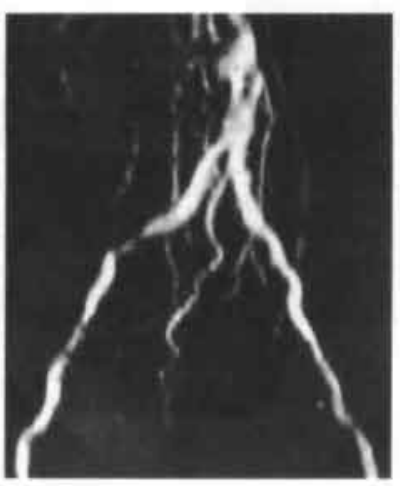

Figure 1. Time-of-flight MR angiographic maximum intensity projection (MIP) images of the pelvic arteries of a 75 -year-old woman. A: Noncardiac synchronized MIP image shows poor image quality due to ghosting artifacts, inplane saturation, slow flow and pre-saturation slab artifacts. B: The same arteries imaged with the same, but systolic cardiac synchronized, time-of-flight MR angiographic technique, shows significantly improved image quality.

If no cardiac synchronization is used, acquiring the phase encoding steps in the anteroposterior direction can prevent ghosting artifacts from being seen on the coronal maximum intensity projection (MIP) image since these artifacts only occur in this time dependent direction. Flow voids, however, can originate from different causes. The main two causes are inplane saturation and post-stenotic turbulence effects. Inplane saturation is caused by an increased intraslice transit time, submitting blood to too many (saturating) RF pulses and thereby making it indistinguishable from background tissue (flow void). Increased intraslice transit times can occur with tortuous vessel courses, thick slices (or the use of three-dimensional (3D) volumes) and low flow velocities. As discussed in the previous section the latter two causes can be prevented using thin(ner) slices and systolic cardiac synchronization, but inplane saturation caused by the course of the vascular tree is more cumbersome. Particularly in regions of the (common) iliac arteries and popliteal trifurcation, the vessels tend not to run straight down (feet-head direction). Therefore the axially planned slices are 
not perpendicular to the vessels and inplane vessel course with resulting inplane saturation occurs .

The other main cause for flow voids, being post-stenotic turbulence is also difficult to prevent. Post-stenotic turbulence directly distal to a stenosis, induces accelerated intravoxel phase dispersion. To prevent this effect the shortest possible TE should be used, sampling the echoes before phase dispersion has faded out signal from a voxel. Since during systole maximum blood flow velocities are present, this is also the window in the heart cycle at which post-stenotic turbulence is worst. As seen in figure 2, when using systolic cardiac synchronization, post-stenotic flow voids are more severe compared with nonsystolic cardiac synchronized techniques.
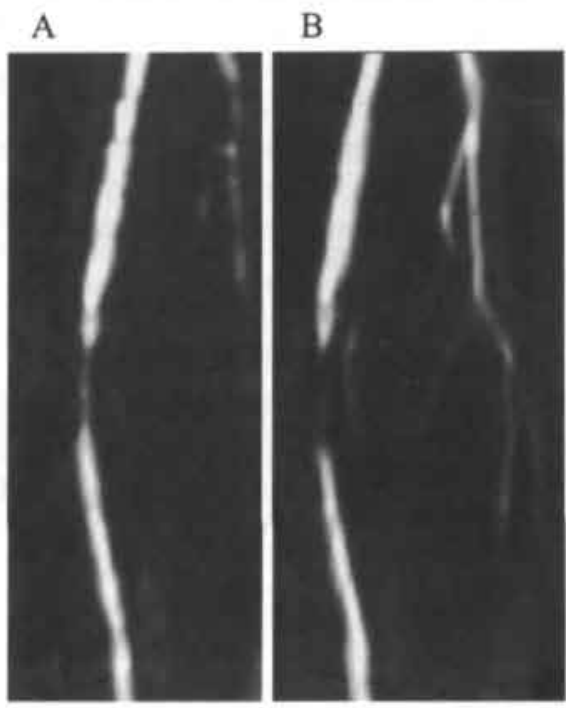

Figure 2. Time-of-flight MR angiographic maximum intensity projection (MIP) images of the femoral arteries of a 58-year-old man. A: MIP image of a noncardiac synchronized time-of-flight MR angiographic technique shows stenosis in the left femoral artery. B: The same artery imaged with the same, but cardiac synchronized, time-of-flight MR angiographic technique shows the stenoses as seen in fig. A, but the stenosis seems to be more severe due to increased poststenotic turbulence and its resulting faster intravoxel phase dispersions.

However, image quality using cardiac synchronization is significantly better [9].

A less common flow void in (noncardiac synchronized) TOF MR angiography can be caused by the venous presaturation slab in combination with a tri-phasic blood flow

pattern. During diastole, retrograde flow of arterial blood, saturated by the venous presaturation slab can occur and gives rise to flow voids. Of course, in cases of retrograde arterial flow, for instance distal to complete occlusions with collateral filling of runoff vessels, flow voids can also be induced by the venous presaturation slab.

A final artifact, easily prevented during acquisition, is caused by slice to slice misalignment of blood signal. If a MIP image is reconstructed, this artifact can mimic stenoses. To prevent this artifact, imaged slices should be acquired overlapping.

As has been noted by many other investigators, TOF MR angiography needs to be evaluated on source images $[15,16]$. Especially in cases where background saturation was inadequate, resulting in poor vessel-to-background contrast, MIP images can lead to erroneous image interpretations.

\section{Validity}

TOF MR angiography has been evaluated fairly extensively for aorta and iliac arteries. Sensitivity and specificity for detection of hemodynamically significant 
stenoses in these arteries ( $250 \%$ reduction in lumen diameter) both ranged from 63 $100 \%$ when either standard or intra-operative diagnostic X-ray angiography was used as the standard of reference [6-10]. Other outcomes which have been used to compare TOF MR angiography with diagnostic X-ray angiography, are agreement between optimal treatment plans formulated on the basis of both imaging techniques and the ability to demonstrate patent runoff vessels below the knee $[8,11]$. Several authors even claim that with proper technique, TOF MR angiography has greater sensitivity than diagnostic X-ray angiography in demonstrating patent runoff vessels in the lower leg region [11,17]. However, in a more recent report this finding could not be confirmed consistently [8].

\section{Contrast-enhanced MR Angiography}

MR angiographic image quality has improved significantly since CE MR angiography was introduced. This technique is based on the property of intravenously injected gadolinium-chelates to reduce bloods $\mathrm{Tl}$, almost eliminating the saturation effect of blood in the imaged slice [18]. Therefore high resolution 3D volumes encompassing the arteries of interest as a whole can be used while acquisition times of less than half a minute are possible. Most sequences used are 3D gradient-recalled echo techniques with heavy T1-weighting. Short TR values ensure good background saturation while high intra-arterial concentration of contrast material ensures good vessel-to-background contrast. T1 reduction of blood is almost directly proportional to the intra-arterial concentration of gadolinium-chelates [18]. Therefore imaging during bolus passage of contrast material is needed to achieve highest intra-arterial signal intensities. The concentration of contrast material in the bolus can be increased by faster intravenous injection of contrast material.

\section{Timing Contrast Injection}

To take full advantage of the passing bolus of contrast material in the imaged volume, the arrival-time of the bolus has to be determined. Arrival-time is defined as the time passed between start of intravenous injection and arrival of contrast material in the volume of interest. For this purpose a timing sequence can be used. This is a dynamic T1-weighted sequence with which one single slice is acquired every 1-2 seconds, consecutively. To determine arrival-time for the pelvic arteries, this slice is best planned proximal to the aortic bifurcation, since planning in the pelvic region can make detecting the (small) arteries very difficult, particularly when they are severely diseased. The use of an axially planned slice is probably easiest and fastest, since no careful positioning in anteroposterior or left-right direction is needed. If an axially planned slice is used, a presaturation slab cranial to the imaged slice should be applied to prevent inflow effects from arterial blood.

A test bolus of 2-mL of contrast material (gadolinium-chelates with a concentration of $0.5-\mathrm{mmol} / \mathrm{mL}$ ) with a subsequent flush of $30-\mathrm{mL}$ of $0.9 \%$ saline (to flush contrast material from tubing and veins), is injected with the same injection rate as planned for the CE MR angiographic scan. The dynamic scan and the injection of the test bolus 
(and subsequent normal saline flush) are started simultaneously. The scanned slices are then evaluated for an increase in signal intensity of the abdominal aorta, representing the arrival of the test bolus. The number of the slice in which the elevated intra-aortic signal intensity is seen, multiplied by the temporal resolution per slice can be used as the arrival-time of the bolus of contrast material in the planned volume. In order to determine the scan-delay time, which is defined as the time passed between start of intravenous injection and start of the contrast-enhanced scan, 5-8 seconds should be added to the arrival-time of the bolus. This is to ensure an even and high enough concentration of contrast material is present in the arteries of interest. Others have used more sophisticated/automated ways to determine scandelay times for CE MR angiography $[19,20]$. However, despite these new developments, determining scan-delay times for lower extremities might prove to be cumbersome because of the small diameter of distal peripheral arteries, dilution effects and differences in arrival-times per leg.

\section{Imaging Parameters}

Since blood is difficult to saturate because of the T1 decreasing effect of gadoliniumchelates, the main limiting factors in CE MR angiography are bolus duration (volume of contrast material used divided by injection rate), time to venous enhancement and respiratory motion (for imaging the upper pelvic arteries). Therefore, contrastenhanced sequences are aimed at acquiring a $3 \mathrm{D}$ coronal volume with a large FOV with highest resolution in the shortest time possible. Maximum FOV (400-500-mm) with a 512 matrix and 150 to 200 phase encoding steps and slice thickness of 2-3$\mathrm{mm}$, ensures fast large coverage and sufficient resolution to image peripheral arteries. Volume thickness should be enough to cover all arteries of interest in the anteroposterior direction. The largest anteroposterior coverage is needed in the pelvic region, which, in almost all patients, does not exceed 100 -mm. If the phase-encoding direction is set from left to right, a rFOV (from left to right) can be used. Since in most patients a width of the rFOV from left to right of $300-\mathrm{mm}$ is sufficient to prevent disturbing wrap-around artifacts, acquisition time can be decreased. Further reduction of acquisition time can be accomplished by using shortest TR and TE values. Depending on the available MR system, TR values can vary from 3-14-msec and TE values from $2-5-\mathrm{msec}$. TR can be reduced to these low values by using partial echo sampling. For CE MR angiography, sampling $60-75 \%$ of the echo is sufficient for adequate results.

\section{Planning the Contrast-enhanced MR Angiographic Sequence}

For planning purposes a fast low resolution noncardiac synchronized TOF MR localizer sequence can be used. Acquiring twenty 4-mm thick slices with an interslice gap of $16-\mathrm{mm}$, covers an area of $400-\mathrm{mm}$ of the vascular tree. A sagittal MIP image of these slices can then be used for planning the contrast-enhanced sequence (fig. 3). 


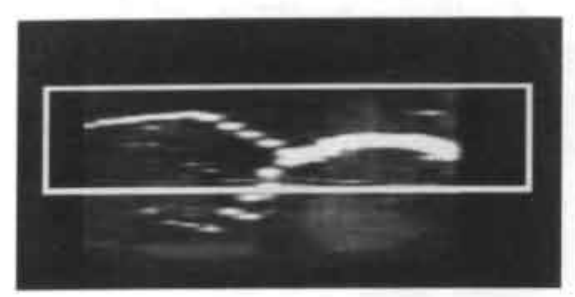

Figure 3. Sagittal maximum intensity projection image of a fast low resolution time-of-flight MR angiographic localizer technique can be used to plan the contrast-enhanced volume. Drawn box represents the three-dimensional planned contrast-enhanced volume.

\section{Acquisition and interpretation pitfalls}

Probably the most common pitfall in CE MR angiography is mistiming arrival of the bolus. As discussed, a timing sequence, either manually or automated can be used to reduce the chance of mistiming. However, even using a timing sequence, mistiming of the bolus can still occur (fig. 4).

A

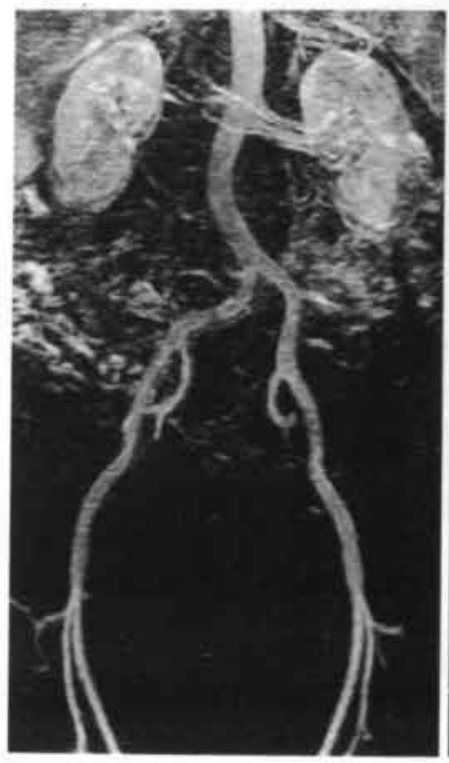

B

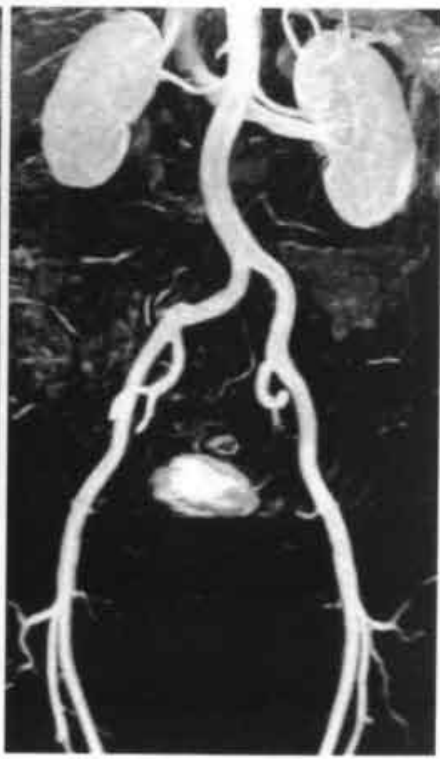

Figure 4. Maximum intensity projection (MIP) images of a 64-yr-old man demonstrating the effect of poor timing of contrast material. A: Poor vesselto-background contrast due to too early acquisition before all arteries contained sufficient concentration of contrast material. B: Same patient with good vessel-tobackground contrast in the MIP as the timing of contrast material was better.

Too early or too late acquisition of the volume of interest results in low intra-arterial signal intensities. Too early acquisition can also result in acquisition of the center Kspace profiles when intra-arterial concentration of contrast material is still increasing, giving rise to a ringing like artifact [21]. Too late acquisition can result in an increased chance of venous enhancement [22].

Interpretation pitfalls mainly consist of artifacts inherent to the use of standard MIP images. These problems can be prevented by using source data, target MIP and multiplanar reformat images (fig. 5). 
A

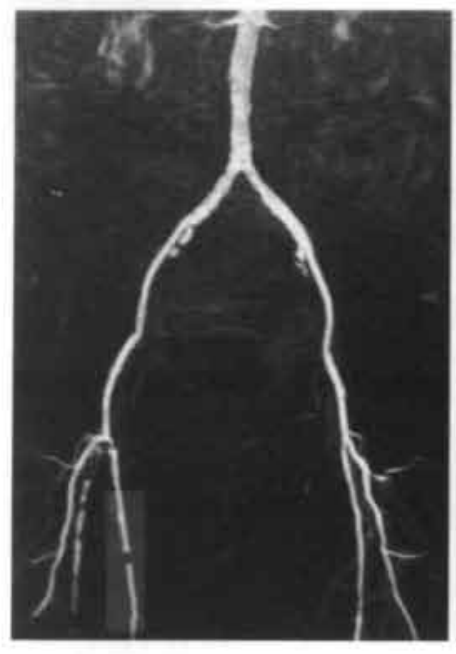

B

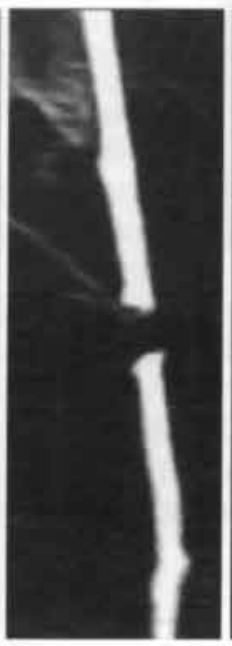

C

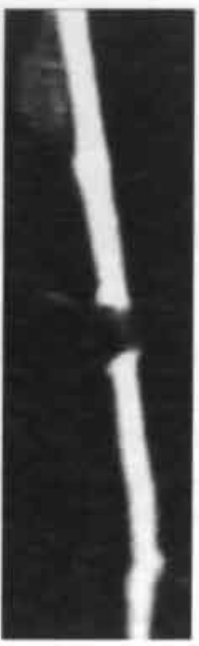

Figure 5. A: Maximum intensity projection (MIP) image of an 84-year-old woman shows a complete occlusion in the right superficial femoral artery (gray box). B: Zoomed MIP image demonstrates the absence of any vascular signal in the area of the occlusion. C: Zoomed target MIP image (all signal anterior and posterior of the vessel eliminated), shows a distinct vascular signal. Therefore, the diagnosis complete obstruction was changed to severe stenosis $(90 \%$ lumen reduction).

\section{Two Methods for Contrast-enhanced MR Angiography}

To date different methods are used for CE MR angiography. A useful classification is by injection and acquisition speed.

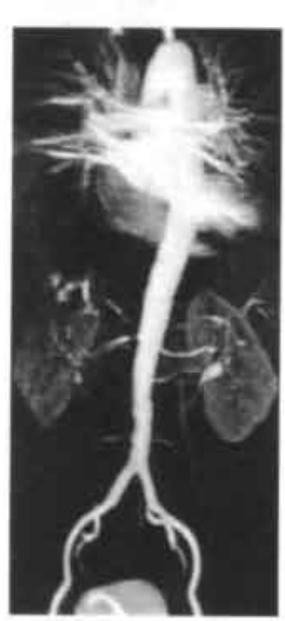

Since fast injection rates $(1-2-\mathrm{mL} / \mathrm{sec})$ lead to relatively short passage times of contrast-enhanced blood, the optimal scan window is short (20-30 seconds). Therefore ultrafast acquisition techniques are required. Ultrashort TR values $(3-8-\mathrm{msec})$ necessary for ultrafast acquisitions, are now possible using the latest commercially available gradient MR systems. As depicted in figure 6 this technique does provide optimum vessel-tobackground contrast [23].

Figure 6. Maximum intensity projection image of a 49-year-old woman shows excellent vessel-to-background contrast using a fast injection rate and fast acquisition technique (1.5-mL/sec, TR/TE 7/3-msec).

However, if ultrashort TR values cannot be achieved, adequate CE peripheral MR angiography is still possible. For this purpose slow injection rates $(0.3-0.5-\mathrm{mL} / \mathrm{sec})$ and acquisition speeds can be used. The slow injection rate technique results in lower intra-arterial concentration of contrast material, inducing less T1 decrease of blood, but it does provide longer bolus duration with equal doses of contrast material compared to the fast injection rate technique. Therefore longer acquisition times can be used, e.g. longer TR values (possible with all commercially available scanners). 
Although longer TR values will give less saturation of blood (hence relatively higher intra-arterial signal intensities), it also gives less background saturation. However, the latter can be compensated for, by using a subtraction technique [24-26]. With this technique the volume of interest is acquired twice. Once before the injection of contrast material and once during the injection of contrast material. Subsequently precontrast volumes are subtracted from contrast-enhanced volumes, resulting in volumes containing preferentially arterial information with excellent vessel-tobackground contrast. Since longer acquisition times are used with this technique, the combination with breath-holding can become more difficult. However, it is our experience that if a patient can hold his breath for $75 \%$ of the acquisition-time, improved image quality can be achieved as seen in figure 7 .

A

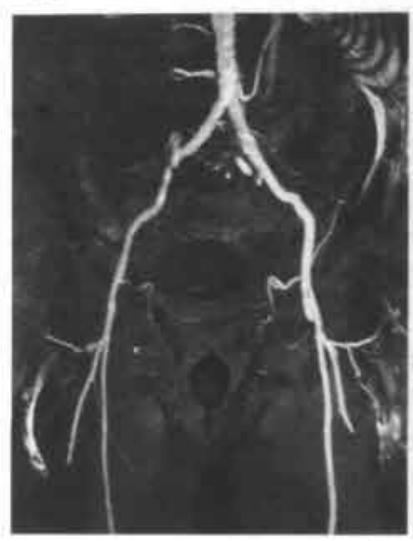

B

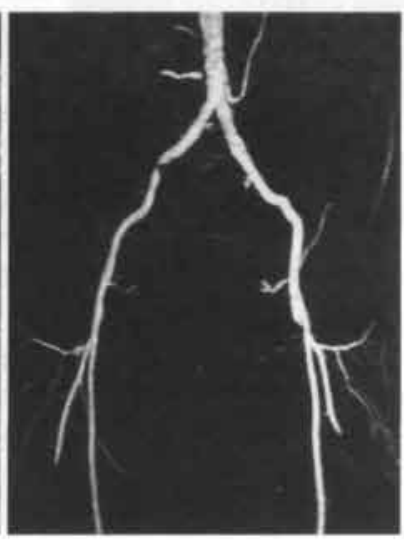

Figure 7. Using a slow injection rate and acquisition technique combined with subtraction and breath holding for (less than) $75 \%$ of the acquisition time (breath holding for $30 \mathrm{sec}$, acquisition time $43 \mathrm{sec}$ ) provides excellent vessel-to-background contrast. A: Nonsubtracted contrast-enhanced maximum intensity projection image shows moderate vessel-to-background contrast. B: Subtracted maximum intensity projection image shows excellent vessel-to-background contrast.

\section{Validity of Contrast-enhanced MR Angiography}

Sensitivity and specificity reported in recent literature for detection of hemodynamically significant stenoses with CE MR angiography ranges from 93$100 \%$ and $89-100 \%$, respectively, for the infra-renal aorta, iliac and common femoral arteries [3,6,7,16,24,25,29-31]. These figures compare favorably with TOF MR angiography. For peripheral runoff vessels only 2 studies (with a total of 43 patients) have been published which have calculated sensitivity and specificity for detection of hemodynamically significant stenoses and these are $79-97 \%$ and $96-100 \%$, respectively $[3,25]$. However, only Ho et al specifically give information for each runoff vessel [3]. Adding multi-planar reformations to MIP images when assessing vessel segments for pathology increases sensitivity of CE MR angiography from $92 \%$ to $96 \%$ [16]. 


\section{Time-of-flight versus Contrast-enhanced MR Angiography}

Although good results for detecting and grading stenoses using TOF MR angiographic techniques have been reported in literature, CE MR angiography has outclassed TOF MR angiography in almost every way imaging the aorta and peripheral arteries (fig. 8).

A

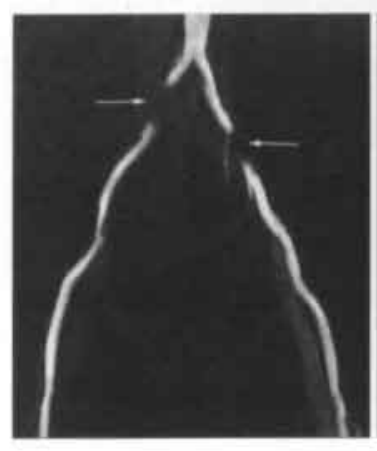

B

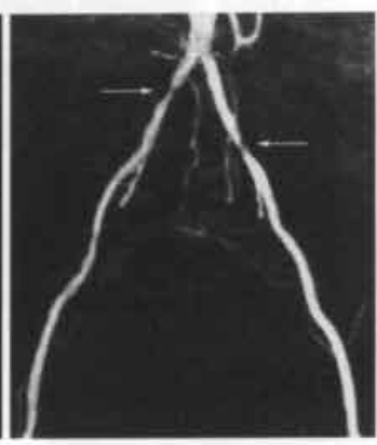

Figure 8. Maximum intensity projection (MIP) images of the pelvic arteries of a 54-year-old woman. A: MIP made from a cardiac synchronized time-of-flight technique shows bilateral complete occlusions of the common iliac arteries (arrows). B: MIP made from a contrast-enhanced technique shows that the bilateral complete occlusions in the common iliac arteries were in fact stenoses (arrows) with lumen reductions of $65 \%$ (right common iliac artery) and $75 \%$ (left common iliac artery).

TOF MR angiography is hampered by the virtue by which it exists. Blood flow ensures the inflow of fresh unsaturated blood creating the arterial signal seen in these images, but blood flow is also responsible for the flow dependent artifacts (poststenotic turbulence, ghosting, etc.). Another disadvantage is the time-consuming nature of this technique (axially planned slices, cardiac synchronization). Imaging the entire peripheral vascular tree can take up to several hours depending on the hardware used. In comparison, with CE MR angiography the entire peripheral vascular tree can be imaged in several minutes with only very few artifacts, which, if familiar with the technique can easily be prevented or detected and usually do not disturb image interpretation.

\section{Utility of Peripheral MR Angiography}

The interpretation of CE MR angiographic images can be similar to that of diagnostic $\mathrm{X}$-ray angiographic images. However, the major advantage is that MR angiographic data can be reconstructed in any desired orientation. This advantage completely eliminates the cumbersome problem of over-projection or insufficient viewing directions, as seen with diagnostic X-ray angiography. Another advantage is, that even using gadolinium-chelates to enhance contrast in MR angiography, this technique can be used for patients with renal failure or a history of anaphylaxis to iodinated contrast agents. Adverse event rates for gadolinium-enhanced MRI are in the order of $0.003-0.01 \%$ in two large series comprising a total of 34,439 patients $[32,33]$. By contrast, in a series of 2475 consecutive patients undergoing intra-arterial digital subtraction angiography there was a small but definite risk of $0.2 \%$ for developing acute renal failure, and an overall adverse event rate of $9.5 \%$ [34]. Not 
surprisingly, patients themselves prefer MR angiography over diagnostic X-ray angiography and suffer less procedure related morbidity [35].

Disadvantage of any MR angiographic technique is its relatively low spatial resolution as compared to diagnostic X-ray angiography. Although not as much as compared to TOF MR angiography, CE MR angiography can suffer from partial volume effects caused by this low spatial resolution. This can result in under- but mainly over-estimation of degree of stenoses. Particularly with high graded stenoses, complete loss of intravascular signal intensity can occur.

\section{Future Developments}

Since the introduction of fast gradient MR systems (gradient strengths up to 25 $\mathrm{mT} / \mathrm{m}$, rise times as low as $200 \mu \mathrm{sec}$ ), CE MR angiography has further evolved. Allowing high resolution acquisitions in breath holding times, these MR scanners ensure excellent diagnostic value of the acquired data.

As hardware components like dedicated extremity coils and new contrast-enhancing drugs like "bloodpool agents" are being developed, image quality can further be improved.

The next challenge, already taken up by Korosec et al with their TRICKS technique, is to incorporate hemodynamic information about the peripheral arterial circulation [28]. If this can be accomplished, MR angiography might fully replace diagnostic X-ray angiography in the diagnostic workup of a patient with peripheral vascular obstructive disease.

\section{Conclusion}

CE MR angiography is to be preferred over TOF MR angiography for imaging the peripheral arteries. Using CE MR angiography the entire peripheral vascular tree can be imaged within several minutes. New developments are in sight and will make CE $\mathrm{MR}$ angiography a true competitor for diagnostic $\mathrm{X}$-ray angiography in imaging the peripheral vascular tree. 


\section{References}

1. Wedeen VJ, Meuli RA, Edelman RR, Geller SG, Frank LR, Brady TJ, Rosen BR (1985) Projective imaging of pulsatile flow with magnetic resonance. Science 230; 946-948.

2. Wedeen VJ, Rosen BR, Chesler D, Brady TJ (1985) MR velocity imaging by phase display. J Comput Assist Tomogr 9: 530-536.

3. Ho KYJAM, Leiner T, de Haan MW, Kessels AGH, Kitslaar PJEHM, van Engelshoven JMA (1998) Peripheral vascular tree stenoses: evaluation with movingbed infusion-tracking MR angiography. Radiology 206: 683-692.

4. Wang Y, Lee HM, Khilnani NM, Trost DW, Jagust MB, Winchester PA, Bush HL, Sos TA, Sostman HD (1998) Bolus-chase MR digital subtraction angiography in the lower extremity. Radiology 207: 263-269.

5. Lee HM, Wang Y (1998) Dynamic k-space filling for bolus chase 3D MR digital subtraction angiography. Magn Reson Med 40: 99-104.

6. Quinn SF, Sheley RC, Semonsen KG, Leonardo VJ, Kojima K, Szumowski J (1998) Aortic and lower-extremity arterial disease: evaluation with MR angiography versus conventional angiography. Radiology 206: 693-701.

7. Quinn SF, Sheley RC, Szumowski J, Shimakawa A (1997) Evaluation of the iliac arteries: comparison of two-dimensional time of flight magnetic resonance angiography with cardiac compensated fast gradient-recalled echo and contrastenhanced three dimensional time of flight magnetic resonance angiography. JMRI 7: 197-203.

8. Huber TS, Back MR, Ballinger RJ, Culp WC, Flynn TC, Kubilis PS, Seeger JM (1997) Utility of magnetic resonance arteriography for distal lower extremity revascularisation. J Vasc Surg 26: 415-424.

9. Ho KYJAM, de Haan MW, Oei TK, Koster D, Kessels AGH, Janevski BK, Kitslaar PJEHM, van Engelshoven JMA (1998) MR angiography of the iliac and upper femoral arteries using four different inflow techniques. AJR Am J Roentgenol 169:45-53.

10. Cambria RP, Kaufman JA, L'Italien GJ, Gertler JP, LaMuraglia GM, Brewster DC, Geller SG, Atamian S, Waltman AC, Abott WM (1997) Magnetic resonance angiography in the management of lower extremity arterial occlusive disease: a prospective study. J Vasc Surg 25: 380-389.

11. Owen RS, Carpenter JP, Baum RA, Perloff LJ, Cope C (1992) Magnetic resonance imaging of angiographically occult runoff vessels in peripheral arterial occlusive disease. N Engl J Med 326: 1577-1581.

12. Snidow JJ, Aisen AM, Harris VJ, Trerotola SO, Johnson MS, Sawchuk AP, Dalsing MC (1995) lliac artery MR angiography: comparison of three-dimensional gadolinium-enhanced and two-dimensional time-of-flight techniques. Radiology 196: 371-378.

13. Carpenter JP, Holland GA, Golden MA, Barker CF, Lexa FJ, Gilfeather M, Schnall MD (1997) Magnetic resonance angiography of the aortic arch. J Vasc Surg 25: 145151.

14. Szumowski J, Simon JH (1992) Proton chemical shift imaging. In: Stark DD, Bradley Jr WG, eds. Magnetic resonance imaging. 2 Ed. Mosby, St.Louis Baltimore Boston, 479-521.

15. Debatin JF, Hany TF (1998) MR-based assessment of vascular morphology and function. Eur Radiol 8: 528-539.

16. Hany TF, Schmidt M, Davis CP, Gohde SC, Debatin JF (1998) Diagnostic impact of four postprocessing techniques in evaluating contrast-enhanced three-dimensional MR angiography. AJR Am J Roentgenol 170: 907-912. 
17. McDermott VG, Meakem TJ, Carpenter JP, Baum RA, Stolpen AH, Holland GA, Schnall MD (1995) Magnetic resonance angiography of the distal lower extremity. Clinical Radiology 50: 741-746.

18. Prince MR. (1994) Gadolinium-enhanced MR aortography. Radiology 191: I55-164.

19. Foo TKF, Saranathan M, Prince MR, Chenevert TL (1997) Automated detection of bolus arrival and initiation of data acquisition in fast, three-dimensional, gadoliniumenhanced, MR angiography. Radiology 203: 275-280.

20. Wilman AH, Riederer SL, King BF, Debbins JP, Rossman PJ, Ehman RL (1997) Fluoroscopically triggered contrast-enhanced three-dimensional MR angiography with elliptical centric view order: application to the renal arteries. Radiology 205: 137-146.

21. Mezrich R. (1995) A perspective on k-space. Radiology 195: 297-315

22. Kopka L, Vosshenrich R, Rodenwaldt J, Grabbe E (1998) Differences in injection rates on contrast-enhanced breath-hold three-dimensional MR angiography. AJR Am J Roentgenol 170: 345-348.

23. Leung DA, McKinnon GC, Davis CP, Pfammatter T, Krestin GP, Debatin JF (1996) Breath-hold, contrast-enhanced, three-dimensional MR angiography. Radiology 201: 569-571.

24. Ho KYJAM, de Haan MW, Kessels AGH, Kitslaar PJEHM, van Engelshoven JMA (1998) Peripheral vascular tree stenoses: detection with subtracted and nonsubtracted MR angiography. Radiology 206: 673-681

25. Rofsky NM, Johnson G, Adelman MA, Rosen RJ, Krinsky GA, Weinreb JC (1997) Peripheral vascular disease evaluated with reduced-dose gadolinium-enhanced MR angiography. Radiology 205: 163-169.

26. Adamis MK, Li W, Wielopolski PA, Kim D, Sax EJ, Kent KC, Edelman RR (1995) Dynamic contrast-enhanced subtraction MR angiography of the lower extremities: initial evaluation with a multisection two-dimensional time-of-flight sequence. Radiology 196: 689-695.

27. Watanabe Y, Dohke M, Okumura A, Amoh Y, Ishimori T, Oda K, Dodo Y (1998) Dynamic subtraction MR angiography: first-pass imaging of the main arteries of the lower body. AJR Am J Roentgenol 170: 357-360.

28. Korosec FR, Frayne R, Grist TM, Mistretta CA (1996) Time-resolved contrastenhanced 3D MR angiography. Magn Reson Med 36: 345-351

29. Hany TF, Debatin JF, Leung DA, Pfammatter T (1997) Evaluation of the aortoiliac and renal arteries: comparison of breath-hold, contrast-enhanced, three-dimensional MR angiography with conventional catheter angiography. Radiology 204: 357-362.

30. Leung DA, Hany TF, Debatin JF (1998) Three-dimensional contrast-enhanced magnetic resonance angiography of the abdominal arterial system. Cardiovasc Intervent Radiol 21: 1-10.

31. Snidow JJ, Johnson MS, Harris VJ, Margosian PM, Aisen AM, Lalka SG, Cikrit DF, Trerotola SO (1996) Three-dimensional gadolinium-enhanced MR angiography for aortoiliac inflow assessment plus renal artery screening in a single breath hold. Radiology 198: 725-732.

32. Murphy KJ, Brunberg JA, Cohan RH (1996) Adverse reactions to gadolinium contrast media: a review of 36 cases. AJR Am J Roentgenol 167: 847-849.

33. Niendorf HP, Haustein J, Cornelius I, Alhassan A, Clauss W (1991) Safety of gadolinium-DTPA: extended clinical experience. Magn Reson Med 22: 222-228.

34. Waugh JR, Sacharias N (1992) Arteriographic complications in the DSA era. Radiology 182: 243-246.

35. Swan JS, Fryback DG, Lawrence WF, Katz DA, Heisey DM, Hagenauer ME, Selzer PM, Jacobson BK (1997) MR and conventional angiography: work in progress toward assessing utility in radiology. Acad Radiol 4: 475-482. 
Chapter 2 


\section{Chapter 3}

\section{MR Angiography of the Iliac and Upper Femoral Arteries using Four Different Inflow Techniques}

Published in the American Journal of Roentgenology 1997; 169: 45-53

Authors: Ho K.Y.J.A.M., de Haan M.W., Oei T.K., Koster D., Kessels A.G.H., Janevski B.K., Kitslaar P.J.E.H.M., van Engelshoven J.M.A. 



\begin{abstract}
Objective: The purpose of this study was to compare two inflow MR angiographic pulse sequences, obtained both with and without systolic synchronization. We also compared these two MR angiographic pulse sequences with conventional angiography.
\end{abstract}

Subjects and Methods: Thirty-one consecutive patients who were scheduled for conventional angiography because of symptomatic atherosclerotic obstructive disease of the iliac or femoral artery underwent MR angiography using four different MR angiography techniques. These techniques consisted of a multiple two-dimensional inversion prepulse gradient-recalled echo technique (turbo "field-echo") obtained with and without systolic synchronization and a multiple two-dimensional gradientrecalled echo technique (fast "field-echo") obtained with and without systolic synchronization. We then compared image quality and our ability to detect and grade degree and length of stenoses, using conventional angiography as the gold standard.

Results: The systolic-synchronized turbo field-echo sequence produced the best results, both objectively and subjectively. Comparing systolic-synchronized turbo field-echo and fast field-echo with conventional angiography regarding detection and grading degree of stenoses, we found no statistically significant differences.

Conclusion: Systolic synchronization proved to be of significant importance for image quality. The systolic-synchronized turbo field-echo pulse sequence proved to be superior to the other three MR angiographic techniques. 
Chapter 3 


\section{Introduction}

The use of MR angiography of the iliac and femoral arteries has attracted increasing interest over the past 10 years. To take full advantage of this new angiography method, an adequate technique for depicting the vessels and accurately assessing the degree and length of a stenosis is important. Several studies have already shown the capability of MR angiography and, in particular MR angiography used with time-offlight techniques, for imaging the peripheral vasculature. Sensitivity and specificity of approximately $90 \%$ in detecting and grading vascular stenoses have been reported [112]. In these studies, different pulse sequences with and without systolic synchronization (SS) have been used, but these various MR angiography techniques have been compared only on a minor scale [1,3,5,13-15].

The purpose of this prospective comparison was to show which inflow MR angiography technique produces the best results in patients with intermittent claudication. Therefore, we compared the image quality and accuracy for detecting and grading of stenoses of four different MR angiography techniques (two different pulse sequences both with and without SS). Conventional angiography served as the gold standard (Fig. 1).

A

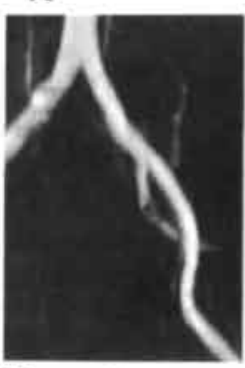

B

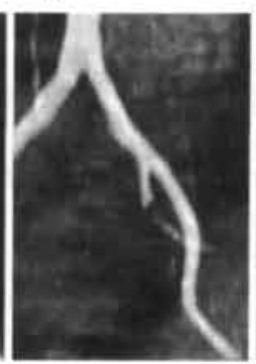

C

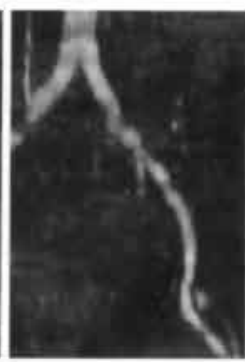

D

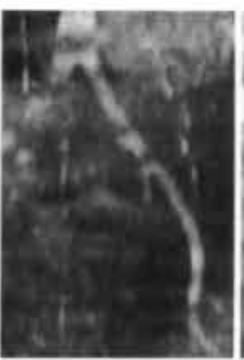

E

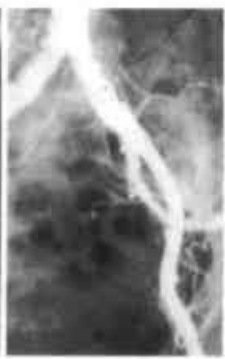

Figure 1. Representative examples of MR angiograms of a 76-year-old woman obtained with four different MR angiography techniques and corresponding conventional angiogram. A: Systolicsynchronized (SS) turbo field-echo (TFE) technique shows high contrast because of high intravascular signal intensities and good background suppression. B: SS fast field-echo (FFE) technique shows high contrast because of high intravascular signal intensities but lower background suppression than SS-TFE technique. C: Non-SS-TFE technique shows lower contrast than SS techniques because of lower intravascular signal intensities. D: Non-SS-FFE technique shows lower contrast than SS techniques because of lower intravascular signal intensities and lower background suppression. E: Corresponding conventional angiogram.

\section{Subjects and Methods}

Thirty-one consecutive patients with intermittent claudication and one or more Doppler sonography-proven stenotic lesions in the iliac or femoral arteries who were scheduled for conventional angiography of the peripheral vessels underwent MR angiography of the iliac or femoral arteries or both using four different inflow techniques. 
The patients were 18 men and 13 women from 45 to 88 years old (mean age, 64 years old). Approval was obtained from the medical ethics commission of the hospital, and written informed consent was obtained from all patients.

\section{MR angiography}

MR angiograms were obtained with a 1.5-T MR system (Gyroscan advanced clinical system-II, release 3.6; Philips Medical Systems, Best, The Netherlands) with a field strength of $10 \mathrm{mT} / \mathrm{m}$ and a slew rate of $10 \mathrm{mT} / \mathrm{m} / \mathrm{ms}$. The body coil was used for signal transmission and reception.

Two different spoiled gradient-recalled echo techniques were used because these techniques offer the best saturation of background tissue available on our system. Both techniques were used with and without SS. The first technique was a multiple two-dimensional (2D) magnetization-prepared gradient-recalled echo technique (turbo "field-echo" [TFE]) acquired with a gated sweep flip angle (variable flip angle up to $50^{\circ}$ ) and preceded by a $180^{\circ}$ inversion prepulse. The $180^{\circ}$ inversion prepulse with a prepulse delay of 250 -msec effectively suppressed signal from surrounding fat. TR and TE were 15-msec and 6.8-msec, respectively. The gated sweep flip angle resulted in equalization of the signal intensity in the imaged slice. The sequence was divided into 6 turbo shots of 30 phase-encoding steps each. Six turbo shots were required to image a single slice.

The second technique was a multiple 2D TFE technique similar to the first technique but with the use of SS. The SS sequence had a trigger delay set to shortest. "Trigger delay" was defined as the time from the detection of the QRS complex (consisting of Q, R, and $\mathrm{S}$ waves) to the measurement of profiles in the middle of $\mathrm{K}$ space. To achieve maximum contrast, the middle of $\mathrm{K}$-space was filled in peak systole. The profiles were thus acquired from 30 to $480-\mathrm{msec}$ after the detection of the QRS complex. Acquiring 100 slices with the non-SS-TFE sequence took $6 \mathrm{~min} 33$ sec and with a patient heart rate of 80 beats per minute, the SS-TFE sequence took 9 $\min 59 \mathrm{sec}$.

The third technique was a multiple 2D magnetization-prepared gradient-recalled echo technique (fast "field-echo" [FFE]). Continuous RF pulses with TR and TE of 22.6-msec and 6.3-msec, respectively, were applied to saturate the background tissue. The fourth technique was a multiple 2D FFE technique similar to the third technique but with the use of SS. The synchronization was achieved by opening a gate in which the echoes were acquired. This technique was optimized for a maximum inflow effect during peak systole by using a gate delay of $150-\mathrm{msec}$ after detection of the QRS complex and a gate width of $250-\mathrm{msec}$, resulting in signal detection during systole. Acquiring 100 slices the non-SS-FFE sequence took $5 \mathrm{~min} 13 \mathrm{sec}$, and with a patient heart rate of 80 beats per minute, the SS-FFE sequence took $15 \mathrm{~min} 47 \mathrm{sec}$.

All four sequences were obtained with a field of view of $250 \times 250-\mathrm{mm}$ and a matrix size of $256 \times 180$, giving an inplane resolution of $1.0 \times 1.4-\mathrm{mm}$. Slice thickness was 5$\mathrm{mm}$ with a 2 -mm overlap and the images were acquired with one signal average. A tracking venous presaturation slab of $100-\mathrm{mm}$ thickness with a slab gap of $5-20-\mathrm{mm}$ caudal to the imaged slice was used to suppress venous signal. The slices were planned so they would cover the stenosis found with Doppler sonography. 
One hundred slices were obtained with the SS-TFE technique for all patients, thus covering a field of view of $300-\mathrm{mm}$. Depending upon time available for scanning, 50 or 100 slices were acquired with the other three techniques, thus covering a field of view of $150-\mathrm{mm}$ and $300-\mathrm{mm}$, respectively. Maximum intensity projections (MIPs) were reconstructed in three directions.

\section{Conventional angiography}

Conventional angiography was performed within two weeks of MR angiography with a diagnostic Arc X-ray system (Philips Medical Systems) with a programmable stepping C-arm, a Puck film-changer (Elema-Schonander, Solina, Sweden), a Technicare DR 960-B add-on digital subtraction angiography system (General Electric Medical Systems, Milwaukee, WI), and a Power injector (MEDRAD, Pittsburgh, PA). All angiograms were supervised by an experienced vascular radiologist. The standard protocol consisted of puncturing the common femoral artery and placing a 5-French catheter in the distal aorta just above the bifurcation if examination of two legs was required or using a crossover approach if examination of only one leg was necessary. A nonionic contrast medium (Omnipaque, Nycomed, Cork, Ireland) was used in variable volumes and flow rates depending on both the location of the tip of the catheter and the imaging system (digital subtraction angiography or conventional angiography). Cut films were obtained in the posteroanterior direction, and in most patients additional posteroanterior and oblique films were obtained using digital subtraction angiography.

\section{Image evaluation}

For evaluation purposes, the arterial tree on the MIP and conventional angiography images was divided into the following segments: aorta, both right- and left-sided common iliac artery, external iliac artery, common femoral artery, deep femoral artery, and superficial femoral artery. Because not all MR angiography and conventional angiography images contained all segments, only the available corresponding imaged segments could be compared.

Image quality of each pulse sequence was analyzed in three different ways: objectively measuring signal intensities and calculating mean signal intensity ratios, subjective assessment of image quality by three observers, and comparing the image interpretation as to the degree and length of a stenosis as assessed by three observers mutually and with conventional angiography as the gold standard. Evaluating a technique, the observers were unaware of the results of the other MR angiography techniques and conventional angiography; when evaluating conventional angiography, they were unaware of MR angiography results. Signal intensity ratios were calculated by dividing the intravascular signal intensity by the signal intensity of stationary tissue. To measure intravascular signal intensities, an operator-dependent region of interest was drawn in each vessel segment not containing stenoses according to the evaluation on conventional angiography. The same was done for a 10 $\mathrm{cm}^{2}$ region of interest of the background tissue. Signal intensity ratios could not be 
calculated on all separate segments of the arterial tree because of flow voids in some vascular segments.

Each MIP was evaluated subjectively by three vascular radiologists using a mean subjective quality scoring scale from $I$ to 10 based on vessel-background contrast and artifacts [16,17]. A score of 10 represented an excellent angiography image comparable with conventional angiography and a score of 1 , a bad image without any vessel depiction. All images were randomly offered to the examiners, who were unaware of the results of the technique used.

The two sequences scoring best on both quality tests as mentioned previously were then further evaluated for interobserver agreement and evaluation accuracy. Image quality was considered too low for further evaluation if the score was equal to or less than 3.5. These images were excluded from further evaluation.

For evaluation, each imaged segment was scored by two vascular radiologists and one vascular surgeon. The scoring was done by assessing the most severe stenosis per vessel segment and categorizing it using a scale from 1 to 5 , where $1=$ no abnormality, 2 = stenosis ranging from $20 \%$ to $50 \%, 3=50 \%$ to $75 \%, 4=75 \%$ to $100 \%$, and $5=$ complete obstruction $[2,4,17]$. In some cases, stenosis percentage could not be determined because of complete signal loss at and distal to the stenosis. In these cases, complete signal loss over a distance smaller than $10-\mathrm{mm}$ was graded as a $50 \%$ to $75 \%$ stenosis (score $=3$ ), and signal loss $210-\mathrm{mm}$ and smaller than 30 $\mathrm{mm}$ was graded as a stenosis ranging from $75 \%$ to $100 \%$ (score $=4$ ). Complete obstruction was defined as complete signal loss $230-\mathrm{mm}$. The length of the stenoses on MR angiography images was defined as the length of intravascular signal void or diminished signal intensity or both. To maximize the accuracy of the measurements, a ruler with markings of $0.5-\mathrm{mm}$ was used. The conventional angiography images were evaluated for the degree and length of a stenosis by three observers and categorized in the same way as on MR angiography. This evaluation was done during separate sessions 4 weeks before the interpretations of MR angiograms. The observers had to reach consensus: if no consensus was reached, a fourth observer decided on the point of disagreement.

The validity of the interpretation of these two MR angiography pulse sequences was determined by comparing the interpretations of the three observers as to the presence of stenotic lesions with the interpretation of conventional angiography as the gold standard. For this purpose, the vascular tree was evaluated for the presence of nondiseased and diseased vessel segments (lumen reduction of $\geq 20 \%$ ) and for vessel segments with and without a hemodynamically significant stenosis (lumen reduction of $250 \%$ ) [3,11]. Moreover, the length in millimeters of stenotic lesions on MR angiography images, as measured by the three observers, were compared mutually and with the length of the corresponding stenoses on conventional angiography as the gold standard.

\section{Statistical analysis}

Pairwise comparison of the signal intensity ratios and the subjective quality scores for the four different techniques was done with the two-tailed t-test. The interobserver agreement for grading the stenosis for the two sequences that were evaluated for the 
presence of stenoses was done using the Kappa statistics ( $\square$ ), and linear weighted kappa values were calculated $[18,19] . \kappa$ can range from -1 (no agreement) to +1 (perfect agreement). $\kappa$ can be interpreted as poor (0), slight $(0-.2)$, fair $(.21-.40)$, moderate (.41-.60), substantial (.61-.80), and almost perfect (.81-1.00) [20]. Sensitivity and specificity of the two best-scoring MR angiography sequences were calculated for the presence of diseased vessel segments (lumen reduction of $\geq 20 \%$ ) and for the presence of hemodynamically significant stenosis (lumen reduction of $250 \%$ ) with conventional angiography as the gold standard and were compared mutually using the McNemar's symmetry test. The relationship between the MR angiography and conventional angiography scores for the length and degree of a stenosis were analyzed using standard linear regression analysis and the Pearson correlation coefficient [7]. The Pearson correlation coefficient is a measure of how two random variables are associated, assuming that these variables have a linear relationship [21]. P-values of less than .05 were considered significant.

\section{Results}

All patients were able to tolerate MR angiography. Of the 31 patients examined with MR angiography, two patients underwent conventional angiography of a different region, and the MR angiography images of these patients were compared mutually only. Of the 29 patients undergoing conventional angiography, 28 had comparable SS-TFE images and 24 had comparable SS-FFE images. Table 1 shows the location, number, and degree of stenotic lesions seen on conventional angiography.

Table 1. Conventional Angiography; Number, Degree and Location of Stenoses in 29 patients.

\begin{tabular}{llllll} 
Vessel Segment & $0 \%$ to $20 \%$ & $20 \%$ to $50 \%$ & $50 \%$ to $75 \%$ & $75 \%$ to $100 \%$ & $100 \%$ \\
\hline Aorta & 9 & 3 & 2 & 0 & 0 \\
CI & 26 & 8 & 3 & 2 & 0 \\
EI & 23 & 11 & 8 & 3 & 1 \\
CF & 29 & 4 & 1 & 0 & 0 \\
PF & 25 & 0 & 1 & 0 & 0 \\
SF & 15 & 3 & 2 & 1 & 5
\end{tabular}

Note. $-\mathrm{Cl}=$ common iliac artery, $\mathrm{EI}=$ external iliac artery, $\mathrm{CF}=$ common femoral artery, $\mathrm{PF}=$ profunda femoris artery, $\mathrm{SF}=$ superficial femoral artery.

In one patient, the SS-TFE technique was not performed because of time limitations. A conventional angiography image and the corresponding representative examples of the four different MR angiography techniques are shown in figure 1. Table 2 shows the mean signal intensity ratio per technique and the corresponding mean subjective quality score. The mean signal intensity ratios and the mean subjective quality scores of the SS-TFE images were both superior to and statistically significantly better than the mean signal intensity ratios and mean subjective quality scores of the SS-FFE images $(p<.001)$. Each SS technique had statistically significant higher subjective quality scores than the non-SS techniques $(\mathrm{p}<.001)$. 
Table 2. Signal Intensity Ratios (SIR) and Mean Subjective Quality Score (SQS) of Four Different MR Angiographic Techniques in 31 patients.

\begin{tabular}{|c|c|c|c|c|}
\hline Technique & $\begin{array}{l}\text { SIR } \\
\text { Mean }\end{array}$ & SD & $\begin{array}{l}\text { SQS } \\
\text { Mean }\end{array}$ & SD \\
\hline SS-TFE & 3.96 & 0.4 & 7.6 & 0.8 \\
\hline SS-FFE & $2.27 \dagger$ & 0.3 & $5.8+$ & 1.5 \\
\hline non-SS-TFE & $1.75+\ddagger$ & 0.3 & $4.3+\ddagger$ & 1.5 \\
\hline non-SS-FFE & $1.71 \dagger \ddagger$ & 0.2 & $3.7+t$ & 1.4 \\
\hline \multicolumn{5}{|c|}{$\begin{array}{l}\text { Note, }-\mathrm{SD}=\text { standard deviation, SS-TFE = systolic "cardiac-synchronized" turbo field-echo MR } \\
\text { angiographic technique, SS-FFE = systolic "cardiac-synchronized" fast field-echo MR angiographic } \\
\text { technique. † Difference from SS-TFE is statistically significant with } \mathrm{p}<.001 \text {, } \neq \text { Difference from SS-FFE is } \\
\text { statistically significant with } p<.005 \text {. }\end{array}$} \\
\hline
\end{tabular}

Because both SS techniques scored highest on both the objective and the subjective quality test, evaluation for interobserver agreement and accuracy was done only with these two techniques. Three of the SS-FFE images had inadequate image quality (mean subjective quality score $\leq 3.5$ ) and were excluded from further evaluation. The kappa values calculated for agreement among the three observers for each technique are shown in Table 3.

Table 3. Interobserver Agreement for Degree of Stenosis on MR Angiographic Images*.

\begin{tabular}{llll} 
Technique & Observer & $\kappa \dagger$ & Confidence interval \\
\hline SS-TFE & 1,2 & 0.76 & $0.66-0.85$ \\
SS-TFE & 1,3 & 0.77 & $0.68-0.86$ \\
SS-TFE & 2,3 & 0.71 & $0.61-0.81$ \\
SS-FFE & 1,2 & 0.87 & $0.78-0.96$ \\
SS-FFE & 1,3 & 0.85 & $0.76-0.94$ \\
SS-FFE & 2,3 & 0.81 & $0.71-0.91$
\end{tabular}

Note.-Twenty-eight patients were examined with the systolic-synchronized (SS) turbo field-echo (TFE) technique (176 vessel segments). Twenty-four patients were examined with the SS fast field-echo (FFE) technique (106 vessel segments). * For patients having both MR angiography and conventional angiography. $+\mathrm{SD}=.05$.

These kappa values were calculated from 176 scoring results for the SS-TFE sequence and from 106 scoring results for the SS-FFE sequence.

Comparing the interpretation of each observer of the MR angiography images, sensitivity and specificity were calculated for visualizing diseased vessel segments (lumen reduction of $\geq 20 \%$ ) and hemodynamically significant stenosis (lumen reduction of $250 \%$ ) with conventional angiography as the gold standard. Table 4 tabulates the results of these calculations. Using the McNemar symmetry test, no statistically significant difference in sensitivity and specificity could be seen between the two techniques.

The Pearson correlation coefficients of the three observers between conventional angiography and MR angiography scores for the degree of stenoses ranged from .61 to $.74(\mathrm{p}<.001)$ for the TFE technique and from .55 to $.59(\mathrm{p}<.001)$ for the FFE technique. Figure 2 correlates the degree of a stenosis on conventional angiography and MR angiography (observer 1, SS-TFE and SS-FFE) using a linear regression analysis. 
Table 4. Sensitivity and Specificity for Predicting Diseased Vessel Segments and Hemodynamically Significant Stenosis on MR Angiography*

\begin{tabular}{llllll} 
Technique & Observer & \multicolumn{2}{l}{$\begin{array}{l}\text { Diseased Vessel Segments } \\
\text { Lumen reduction of } 20 \% \\
\text { Sensitivity } \\
(\%)\end{array}$} & $\begin{array}{l}\text { Specificity } \\
(\%)\end{array}$ & $\begin{array}{l}\text { Hemodynamically Significant Stenosis } \\
\text { Sumen reduction of 50\% } \\
\text { Sensitivity } \\
\text { Specificity }\end{array}$ \\
\hline SS-TFE & I & 71 & 84 & 71 & $(\%)$ \\
SS-TFE & 2 & 65 & 89 & 64 & 88 \\
SS-TFE & 3 & 69 & 92 & 68 & 89 \\
SS-FFE & 1 & 66 & 86 & 74 & 86 \\
SS-FFE & 2 & 56 & 88 & 63 & 89 \\
SS-FFE & 3 & 63 & 91 & 74 & 89
\end{tabular}

Note.- Sensitivity and specificity were calculated from data of 28 patients who underwent the systolicsynchronized (SS) turbo field-echo (TFE) technique and 24 patients who underwent the SS fast field-echo (FFE) technique. * Using conventional angiography as the gold standard.

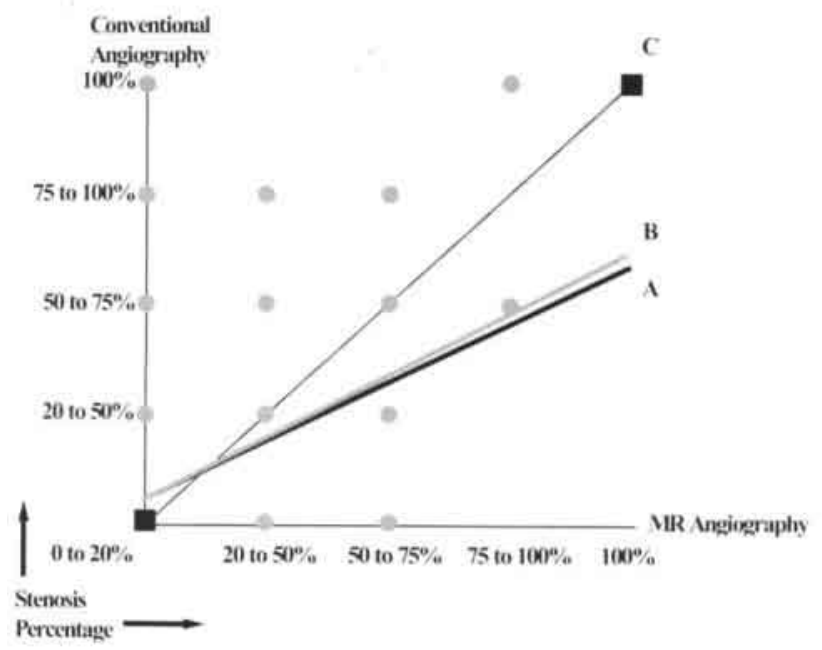

Figure 2. Graph of linear regression analysis for systolic-synchronized (SS) turbo field-echo (TFE) and SS fast fieldecho (FFE) MR angiography as inter-preted by observer 1 shows degree of stenosis revealed on conventional angiography. Note that both MR angiography techni-ques tend to overestimate degree of stenosis compared with conventional angiography because of many false-positive findings when conventional angiography shows no disease. " $\mathrm{A}$ " is linear regression line calculating conventional angiography stenosis percentage $(0.64+0.54 \times$ SS-TFE, $p<.001)$. " $\mathrm{B}$ " is linear regression line calculating conventional angiography stenosis percentage $(0.67+0.55 \times$ SS-FFE, $p<.001)$. Line " $\mathrm{C}$ " shows $\mathrm{MR}$ angiography = conventional angiography.

The same was done for the other observers, but the results did not have a statistically significant difference.

To give a better view of the (dis)similarity between the scores of MR angiography and conventional angiography for the degree of a stenosis, Table 5 gives the crosstabulation of the scores of MR angiography (observer 1, SS-TFE) and conventional angiography. Table 6 tabulates, for all vessel segments and for diseased vessel segments (lumen reduction of $\geq 20 \%$ on conventional angiography), the percentages of overestimations and underestimations of the degree of stenoses found on MR angiography (observer 1 , SS-TFE). 
Table 5. Cross-tabulation of Percentages of Stenoses between SS-TFE (Observer 1) and Conventional Angiography.

\begin{tabular}{llllll}
$\begin{array}{l}\text { SS-TFE } \\
\text { (\%) }\end{array}$ & 0 to 20 & 20 to 50 & 50 to 75 & 75 to 100 & 100 \\
\hline 0 to 20 & 102 & 8 & 6 & 0 & 0 \\
20 to 50 & 6 & 6 & 2 & 0 & 0 \\
50 to 75 & 8 & 4 & 4 & 5 & 1 \\
75 to 100 & 2 & 2 & 4 & 0 & 1 \\
100 & 3 & 0 & 0 & 1 & 4
\end{tabular}

Note.-High number of underestimation of degree of stenosis by MR angiography occurs when normal vessel segments ( $0 \%$ to $20 \%$ ) on conventional angiography are excluded from this cross-tabulation. SS $=$ systolic-synchronization, TFE $=$ turbo field-echo.

Table 6. Overestimations and Underestimations of Degree of Stenosis on MR angiography* as Found in All Vessel Segments and in Diseased Vessel Segments.

\begin{tabular}{lllllll} 
& \multicolumn{2}{l}{$\begin{array}{l}\text { All Vessel Segments } \\
\text { Over- } \\
\text { estimation(\%) }\end{array}$} & $\begin{array}{l}\text { Under- } \\
\text { estimation(\%) }\end{array}$ & No, & $\begin{array}{l}\text { Diseased Vessel Segments } \begin{array}{l}\text { Over- } \\
\text { estimation(\%) }\end{array} \\
\text { Vessel Segment } \\
\text { estimation(\%) }\end{array}$ & No. \\
\hline aorta & 9 & 27 & 11 & 20 & 60 & 5 \\
CI & 14 & 19 & 37 & 15 & 54 & 13 \\
EI & 22 & 17 & 36 & 23 & 46 & 13 \\
CF & 12 & 15 & 34 & 0 & 83 & 6 \\
PF & 12 & 4 & 26 & 0 & 100 & 1 \\
SF & 36 & 4 & 25 & 50 & 10 & 10 \\
total & 18 & 14 & 169 & 23 & 48 & 48
\end{tabular}

Note.--No. $=$ number of evaluated vessel segments, $\mathrm{Cl}=$ common iliac artery, $\mathrm{EI}=$ external iliac artery, $\mathrm{CF}$ = common femoral artery, $\mathrm{PF}=$ profunda femoris artery, $\mathrm{SF}=$ superficial femoral artery. * Using conventional angiography as the gold standard. - Lumen reduction of $220 \%$ on conventional angiography.

The length of the stenoses (lumen reduction of $220 \%$ ) on conventional angiography varied from 1 to $40-\mathrm{mm}$, and the length of measurements of these lesions on MR angiography (SS-TFE) varied among the observers with a mean difference between observer 1 and 2 of $0.6 \pm 3.8-\mathrm{mm}$, between observer 1 and 3 of $0.2 \pm 3.7-\mathrm{mm}$, and between observer 2 and 3 of $0.4 \pm 2.6-\mathrm{mm}$. Figure 3 shows the correlation between MR angiography (observer 1, SS-TFE and SS-FFE) and conventional angiography measurements of the length of stenoses.

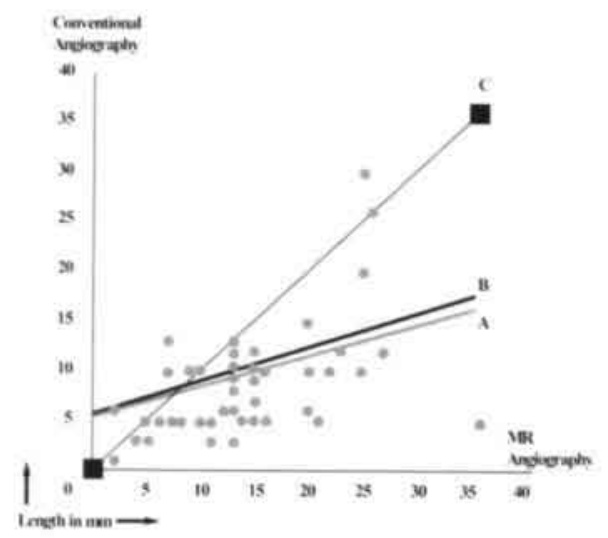

Figure 3. Graph of linear regression analysis for systolic-synchronized (SS) turbo field-echo (TFE) and (SS) fast field-echo (FFE) MR angiography as interpreted by observer 1 shows length of stenosis revealed on conventional angiography. Note that both MR angiography techniques tend to overestimate length of stenosis. " $\mathrm{A}$ " is linear regression line calculating conventional angiographic stenosis length in $\mathrm{mm}(4.7-\mathrm{mm}+0.30 \times$ SS-TFE in $\mathrm{mm}$, $p<.002)$. " $B$ " is linear regression line calculating conventional angiography stenosis length in $\mathrm{mm}(4.2-\mathrm{mm}+0.38 \times$ SS-FFE in $\mathrm{mm}$, $\mathrm{p}<.02)$. Line " $\mathrm{C}$ " shows MR angiography = conventional angiography. 
The Pearson correlation coefficient between conventional angiography and SS-TFE scores of observer 1 was $.47(p=.002)$. When the length of the stenoses found on the SS-TFE images (observer 1) was compared with the length of the corresponding stenosis on conventional angiography images, the overall mean percentage of overestimation of the length was $77 \%$ with a SD of $115 \%$; overall, the overestimation ranged from a minimum of $-67 \%$ to a maximum of $620 \%$, with a median of $44 \%$. Comparing lengths from the SS-FFE images (observer 1) with corresponding stenoses on conventional angiography images, the overall mean percentage of overestimation of the length was $74 \%$ with a SD of $97 \%$; overall, the overestimation ranged from a minimum of $-67 \%$ to a maximum of $333 \%$, with a median of $64 \%$.

\section{Discussion}

The goal of our study was to compare two multiple 2D TOF MR angiography pulse sequences (TFE and FFE) with and without SS in the pelvic and upper femoral region. The results clearly show the importance of SS and the supremacy of the SSTFE technique, although both SS-synchronized pulse sequences tend to overestimate the degree and length of a stenosis more than do the non-SS techniques.

The right technique can significantly improve the image quality of inflow MR angiography of the iliac and upper femoral arteries. The use of SS appears to be essential for both pulse-sequences (TFE and FFE), as shown by the large and statistically significant differences in objective and subjective quality scores achieved with and without SS. The differences in image quality between the TFE and FFE pulse sequences were less impressive, but when these sequences were combined with SS, the differences were also statistically significant.

For our objective quality score, we determined only the signal intensity ratios. We did not calculate the contrast-to-noise ratios because most images did not contain pure noise (field of view of 250-mm covered only body tissue). Because of the lack of signal intensity in stenotic vessel segments, we used only signal intensity ratios of vessel segments that were evaluated on conventional angiography as containing no disease. The results from the objective quality assessment reflected the overall impression of the observers, who also concluded that the use of SS dramatically improved image quality.

Our results concerning the superiority of SS and the TFE pulse sequence in achieving an optimal image quality are also supported by other investigators $[15,16]$. Yucel et al [1] published a similar study of healthy volunteers with the same results concerning image quality. SS prevents artifacts caused by the large variations in flow velocities across the cardiac cycle (fig. 4), vessel pulsatility, and diastolic backflow of saturated blood from the presaturation slab in cases of vessels with a triphasic waveform [1,6,10,22-25] (fig. 5). With non-SS techniques, the image deteriorates because of these three causes. The so-called banding artifact $[22,25]$ can also be prevented in the non-SS techniques by increasing the slice presaturation slab gap. We used a slice slab gap of $20-\mathrm{mm}$ with the FFE pulse sequence and of 5-8-mm with the TFE pulse sequence. This smaller slice slab gap in the TFE pulse sequence is essential to prevent the visualization of the veins. 
A

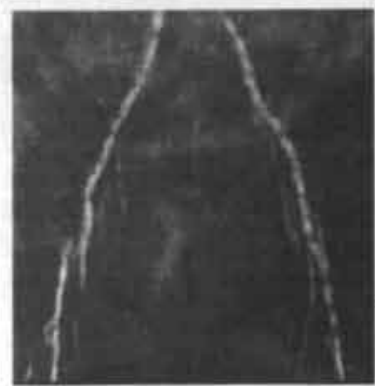

B

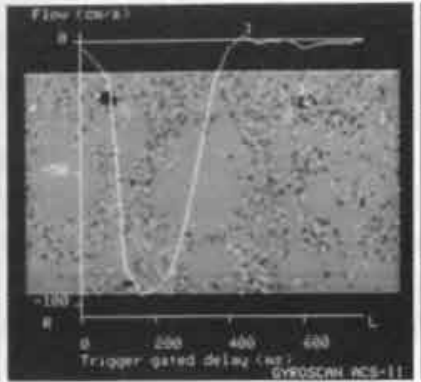

C

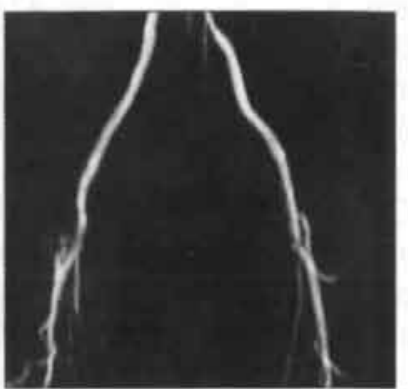

Figure 4. MR angiograms of a 62 -year-old man show that image deterioration occurs in non-systolicsynchronized (SS) time-of-flight MR angiography because of large variations in flow velocities across the cardiac cycle. A: Image deterioration is seen in non-SS turbo field-echo (TFE) MR angiography. B: Time intensity curve (two-dimensional phase contrast angiography) shows almost monophasic waveform of blood flow with fast systolic blood flow acceleration and deceleration in this patient. C: Dramatic improvement in image quality occurs when using SS-TFE technique.

A

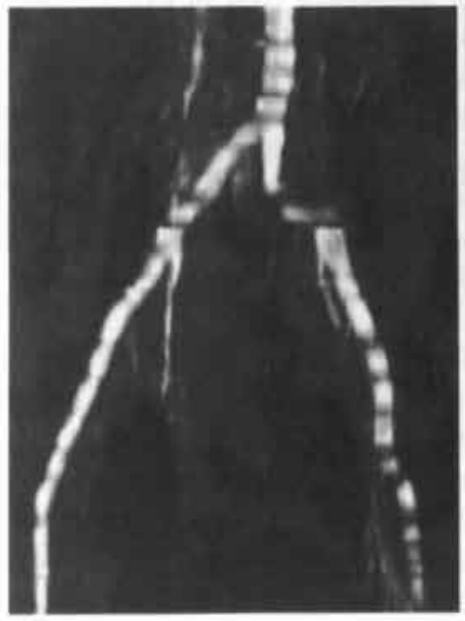

B

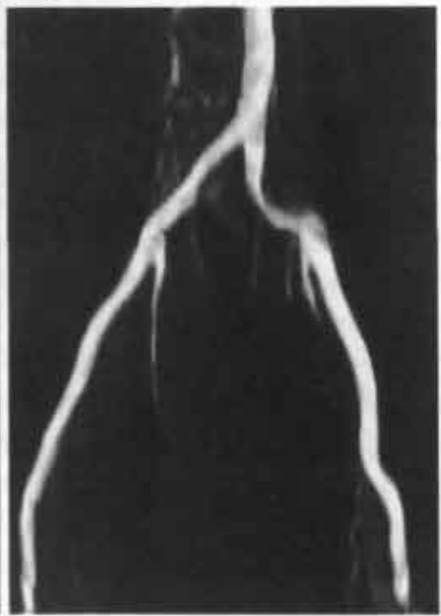

Figure 5. Turbo fieldecho (TFE) MR angiograms of a 66-year-old woman show effect of diastolic backflow on non-systolic synchronized (SS) time-of-flight MR angiography. A: Diastolic backflow causes banding artifacts with non-SS-TFE technique. B: No banding artifact is seen when SS-TFE technique is used.

The $250 \mathrm{msec}$ prepulse delay time used with the TFE sequence will allow the saturated venous blood to recover slightly. Reducing the slice slab gap will help reduce this problem [1]. If a TOF technique is used in an artery that has a poststenotic dampening $[1,6,10,13,24,25]$ of the triphasic waveform, it might be possible to acquire adequate images without $\mathrm{SS}$ and within a shorter time $[1,2,8,26,27]$.

The penalty for SS is acquisition time. With a heart rate of 70 to 80 beats per min, the SS-TFE technique will take double the time needed for the non-SS technique. Because the acquisition time in the SS-FFE technique is shorter per heartbeat than in the SS-TFE technique, scan time for the SS-FFE technique compared with the nonSS-FFE technique will even be tripled. However, the results of the non-SS pulse sequences are unpredictable. We made a time intensity curve $50-\mathrm{mm}$ below the stenoses in all patients, but even with these curves no accurate prediction of the appearance of artifacts or image quality of the non-SS pulse sequences could be 
made. The non-SS techniques produced images that were not suited for further evaluation in $48 \%$ (TFE) and $69 \%$ (FFE) of the examinations. Three of the SS-FFE studies but none of the SS-TFE studies had to be excluded because of too low image quality (mean subjective quality score, s3.5). This better overall image quality of the TFE techniques is due to the better background suppression induced by the $180^{\circ}$ prepulse. This prepulse effectively suppresses signal from surrounding fat. The other advantage of the TFE technique is the gated sweep flip angle that ensures a homogeneous signal from the tissue during the acquisition time. Figure 6 shows the average gain in image quality between the SS-TFE and the SS-FFE technique in favor of the SS-TFE technique despite the more systolic-oriented acquisition window with the SS-FFE technique.

A

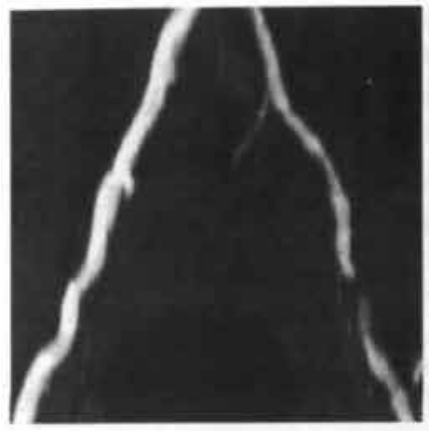

B

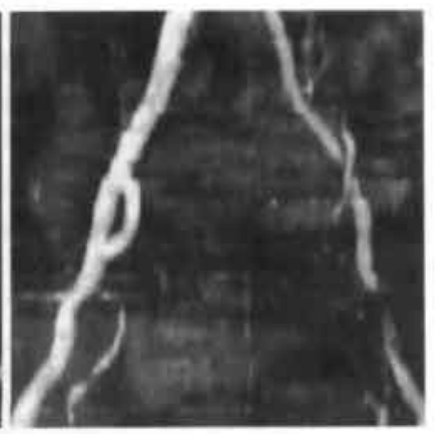

Figure 6. Turbo field-echo (TFE) and fast field-echo (FFE) MR angiograms of a 70-year-old man show improvement in background suppression because of $180^{\circ}$ prepulse with TFE technique. A: Systolic--synchronized (SS) TFE MR angiogram with low background signal intensities. B: SS-FFE MR angiogram with higher background signal intensities.

Because of this better image quality and shorter acquisition time, we prefer the SSTFE to the SS-FFE pulse sequence in patients with intermittent claudication, provided the diagnostic value does not decrease. Therefore, we compared the consistency of image interpretation and the diagnostic value of both techniques using three observers and conventional angiography as the gold standard. The consistency of the interpretation of the grade of a stenosis was high with both techniques and showed no statistically significant differences, which is in agreement with other studies $[28,29]$ and is mandatory to achieve an acceptable test accuracy. The consistency of length measurements was also good. Lengths of stenoses on conventional angiography, however, were all smaller than 40-mm. Accurate stenosis length measurements of such short stenoses-are not important in the peripheral vascular system. For treatment planning, it is important only to differentiate short from long ( $>70-\mathrm{mm})$ stenoses or occlusions.

The sensitivities and specificities of both techniques, although low in comparison with those reported in the literature, did not show a statistically significant difference; therefore, the SS-TFE technique seems to be the best method for MR angiography of the pelvic and femoral arteries.

However, our results concerning the accuracy of MR angiography differ from those of Yucel et al [1] and Glickerman et al [12]. Both groups of researchers found values for sensitivity of $89 \%$ and $88 \%$, respectively, and for specificity of $92 \%$ and $98 \%$, respectively, in diagnosing hemodynamically significant stenosis in the pelvic and 
femoral arteries. This better accuracy might be caused by different interpretation criteria, thinner slices, and the use of axial slices to assess the degree and length of a stenosis, in stead of interpreting three MIPs only, as we did. However, they did not determine the consistency of their image interpretations.

Also, our sensitivity to diagnose occlusion was relatively low. Cross-tabulation in Table 5 shows that observer 1 missed two and erroneously diagnosed four occlusions, which resulted in a sensitivity and specificity for diagnosing occlusion of $66 \%$ and $98 \%$, respectively. The false-positive results were caused by misinterpretation of vessel anatomy, which happened to all observers and with both pulse sequences. This misinterpretation might indicate that more than three projections are necessary for optimal image interpretation. The false-negative findings were caused by our definition of stenosis and occlusion. Flow voids of less than 30-mm in length were defined as stenosis where as those "stenoses" were occlusions on the conventional angiography images. For therapeutic decisions, it is important only to differentiate short from long (>70-mm) occlusions, because short occlusions can be treated with percutaneous transluminal angioplasty, whereas bypass surgery is the treatment of choice for long occlusions [30].

The SS-techniques exaggerate the poststenotic turbulence-induced intravoxel phase dispersion and the intrastenotic outflow effect $[9,10,22,23,31]$. Figure 7 (SS-TFE and SS-FFE) shows this phenomenon.

A

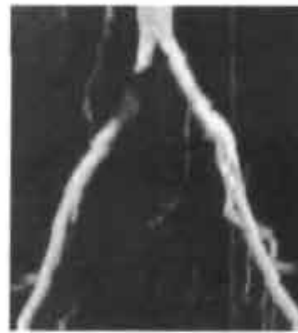

B

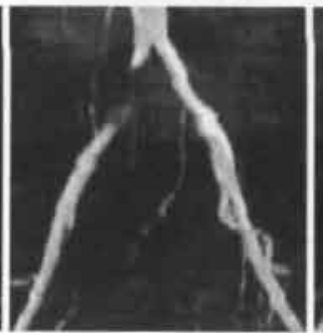

C

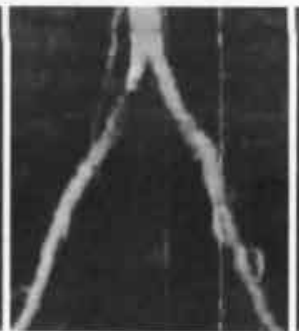

D

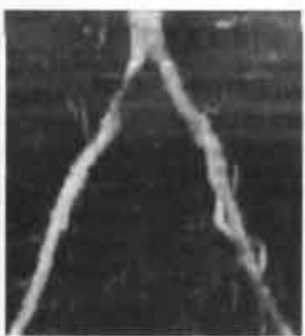

Figure 7. MR angiograms of a 53-year-old man show exaggeration of intra- and poststenotic outflow and turbulence effects when using systolic-synchronized (SS) time-of-flight techniques. A: SS turbo field-echo (TFE) MR angiogram with almost complete signal loss intra- and poststenotically. B: SS fast field-echo (FFE) MR angiogram with even more intrastenotic signal loss than SS-TFE technique because of a more systolic-oriented acquisition window. C: non-SS-TFE MR angiogram with only decreased intrastenotic signal intensity. D: non-SS-FFE MR angiogram with only decreased intrastenotic signal intensity.

Both non-SS images show a shorter length of diminished signal intensity.

A closer correlation between the interpretations of MR angiography (TFE and FFE) and conventional angiography as to degree and length of a stenosis using linear regression analysis (figs. 2 and 3) indicates that both MR angiography techniques tend to overestimate the degree and length of a stenosis, which increases with a higher degree or length of a stenosis on conventional angiography. It was not possible to compare non-SS and SS pulse sequences for the degree of a stenosis because of the small number of interpretable cases using the non-SS technique, but we believe that the non-SS pulse sequences visualized the degree of a stenosis more realistically and with less overestimation (fig. 7). This conclusion can also be reached by comparing 
our results with those of Hertz et al [7], who found a better correlation $(r=.83$, p $<.001$ ) between conventional angiography and MR angiography with less tendency of overestimation using a non-SS technique.

If normal vessel segments on conventional angiography are excluded from Table 5 (retrospectively), the number of underestimations surpasses the number of overestimations. Table 6 shows that in diseased vessel segments, MR angiography underestimates the degree of a stenosis more often than it overestimates. The trend seems to be that this occurs more often in larger vessels than in smaller ones. This phenomenon can also be deduced from data of other investigators [1,2], but until now only Schnall et al [6] mentioned this phenomenon. The many false-positive results found with MR angiography when conventional angiography was normal could be explained by the high sensitivity of the TOF techniques for intravoxel phase dispersion and inplane saturation. The underestimations could be explained by the way the images are obtained. The coronal and sagittal MIPs are made of a stack of transverse slices with high resolution in the transverse plane $[22,32]$. The coronal and sagittal films have considerably lower resolution because of the 5-mm slice thickness.

All patients with symptomatic arteriosclerotic peripheral vessel disease need an extensive diagnostic workup, including some noninvasive tests, to make an accurate diagnosis. Precise anatomic knowledge of the vascular system is mandatory to plan treatment and to decide what type of intervention (percutaneous transluminal angiography, bypass surgery or amputation) is most appropriate. For that reason, the vascular surgeon and the interventional radiologist need an overview of the entire vascular system, including the status of the arterial inflow tract and distal runoff vessels, the location of stenoses and occlusions, the presence of collateral vessels, and so on. The exact degree and length of a stenosis are less important. MR angiography gives the opportunity to provide all this information noninvasively. However, because the non-SS MR angiography technique often fails to acquire images with enough quality for further evaluation SS seems to be mandatory for imaging the iliac and upper femoral arteries, preferably combined with the TFE pulse sequence.

In conclusion. SS is essential for high image contrast and image quality in inflow MR angiography of the pelvic and upper femoral vessels. The faster image acquisition time plus the better image contrast makes the SS-TFE technique superior to the SS-FFE technique.

Acknowledgments: we gratefully acknowledge the assistance of $\mathrm{H}$. Schoenmakers and the other MR technicians of the Radiology department. 


\section{References}

1. Yucel EK, Silver MS, Carter AP. MR angiography of normal pelvic arteries: comparison of signal intensity and contrast-to-noise ratio for three different inflow techniques. AJR 1994;163:197-201.

2. Yucel EK, Kaufman JA, Geller SC, Waltman AC. Atherosclerotic occlusive disease of the lower extremity: prospective evaluation with two-dimensional time-of-flight MR angiography. Radiology 1993;187:637-641.

3. Carpenter JP, Owen RS, Holland GA, et al. Magnetic resonance angiography of the aorta, iliac, and femoral arteries. Surgery 1994;116:17-23.

4. Yoshikawa K, Sugimura K, Kawamitsu H, Ishida T. Intrapelvic two-dimensional time-of-flight magnetic resonance angiography in healthy and diseased subjects. $\mathrm{Br} \mathrm{J}$ Radiol 1994;67:140-146.

5. Carpenter JP, Baum RA, Pentecost MJ, Holland GA, Barker CF. Peripheral vascular surgery with magnetic resonance angiography as the sole preoperative imaging modality. J Vasc Surg 1994;20:861-869.

6. Schnall MD, Holland GA, Baum RA, Cope C, Schiebler ML, Carpenter JP. MR angiography of the peripheral vasculature. Radiographics 1993;13:920-930.

7. Hertz SM, Baum RA, Owen RS, Holland GA, Logan DR, Carpenter JP. Comparison of magnetic resonance angiography and contrast arteriography in peripheral arterial stenosis. Am J Surg 1993;166:112-116.

8. Yucel EK, Dumoulin CL, Waltman AC. MR angiography of lower-extremity arterial disease: preliminary experience. J Magn Reson Imaging 1992;2:303-309.

9. Schiebler ML, Listerud J, Holland G, Owen R, Baum R, Kressel HY. Magnetic resonance angiography of the pelvis and lower extremities. Works in progress. Invest Radiol 1992;27 (Suppl 2):S90-S96.

10. Yucel EK. Magnetic resonance angiography of the lower extremity and renal arteries. Semin Ultrasound CT MR 1992;13:291-302.

11. Carpenter JP, Owen RS, Baum RA, et al. Magnetic resonance angiography of peripheral runoff vessels. J Vasc Surg 1992;16:807-813.

12. Glickerman DJ, Obregon RG, Schmiedl UP, et al. Cardiac-gated MR angiography of the entire lower extremity: A prospective comparison with conventional angiography. AJR;167:445-451.

13. de Graaf RG, Groen JP. MR angiography with pulsatile flow. Magn Reson Imaging 1992;10:25-34.

14. Selby K, Saloner D, Anderson CM, Chien D, Lee RE. MR angiography with a cardiac-phase-specific acquisition window. J Magn Reson Imaging 1992;2:637-643.

15. Lanzer P, Bohning D, Groen J, Gross G, Nanda N, Pohost G. Aortoiliac and femoropopliteal phase-based NMR angiography: a comparison between FLAG and RSE. Magn Reson Med 1990;15:372-385.

16. Lanzer P, Gross G, Nanda N, Pohost G. Timing of data acquisition determines image quality in femoropopliteal phase-sensitive MR angiography. Angiology 1990;41:817824.

17. Walstra BRJ. Arteriography of the lower leg: A comparative study between conventional arteriography and intraarterial digital subtraction arteriography. Datawyse Universitaire Pers Maastricht 1993:90-95.

18. Cohen J. Weighted Kappa: Nominal scale agreement with provision for scaled disagreement or partial credit. Phychol Bull 1968;70:213-230

19. Cohen J. A Coefficient of agreement for nominal scales. Educ Psychol Meas 1960;20:37-46.

20. Landis JR, Koch GG. The measurement of observer agreement for categorical data. Biometrics 1977;33:159-174. 
21. Kleinbaum DG, Kupper LL, Muller KE. Applied regression analysis and other multivariable methods. 2nd ed. Boston: PWS-Kent, 1988: 80.

22. Schiebler ML, Listerud J, Baum RA, et al. Magnetic resonance arteriography of the pelvis and lower extremities. Magn Reson Q 1993;9:152-187.

23. Stahlberg F, Ericsson A, Nordell B, Thomsen C, Henriksen O, Persson BR. MR imaging, flow and motion. Acta Radiol 1992;33:179-200.

24. Caputo GR, Higgins CB. Magnetic resonance angiography and measurement of blood flow in the peripheral vessels. Invest Radiol 1992;27 (Suppl 2):S97-S102.

25. Caputo GR, MasuiT, Gooding GA, Chang JM, Higgins CB. Popliteal and tibioperoneal arteries: feasibility of two-dimensional time-of-flight MR angiography and phase velocity mapping. Radiology 1992;182:387-392.

26. Owen RS, Carpenter JP, Baum RA, Perloff LJ, Cope C. Magnetic resonance imaging of angiographically occult runoff vessels in peripheral arterial occlusive disease. $\mathrm{N}$ Engl J Med 1992;326:1577-1581.

27. Owen RS, Baum RA, Carpenter JP, Holland GA, Cope C. Symptomatic peripheral vascular disease: selection of imaging parameters and clinical evaluation with MR angiography. Radiology 1993;187:627-635.

28. Laissy JP, Limot O, Henry Fuegeas MC, et al. Iliac artery patency before and immediately after percutaneous transluminal angioplasty: Assessment with time-offlight MR angiography. Radiology 1995;197:455-459.

29. Snidow JJ, Aisen AM, Harris VJ, et al. lliac artery MR angiography: Comparison of three-dimensional gadolinium-enhanced and two-dimensional time-of-flight techniques. Radiology 1995;196:371-378.

30. Strecker EP, Boos IBL, Hagen B. Flexible tantalum stents for the treatment of iliac artery lesions: Long-term patency, complications, and risk factors. Radiology 1996;199:641-647.

31. Sebok NR, Sebok DA, Wilkerson D, Mezrich RS, Zatina M. In-vitro assessment of the behavior of magnetic resonance angiography in the presence of constrictions. Invest Radiol 1993;28:604-610.

32. Anderson CM, Saloner D, Tsuruda JS, Shapeero LG, Lee RE. Artifacts in maximumintensity-projection display of MR angiograms. AJR 1990;154:623-629. 
Chapter 3 


\section{Chapter 4}

\section{Peripheral Vascular Tree Stenoses: Detection with Subtracted and Nonsubtracted MR angiography}

Published in Radiology 1998; 206:673-681

Authors: Ho K.Y.J.A.M., de Haan M.W., Kessels A.G.H., Kitslaar P.J.E.H.M., van Engelshoven J.M.A. 



\begin{abstract}
Purpose: To compare subtracted and nonsubtracted gadolinium-enhanced magnetic resonance (MR) angiography and cardiac-synchronized time-of-flight MR angiography for help in detecting pelvic-region stenoses.

Materials and Methods: Twenty-eight patients with intermittent claudication underwent MR angiography with a 1.5-T system; two-dimensional cardiacsynchronized time-of-flight MR angiograms and three-dimensional MR angiograms (without and with gadolinium enhancement) were obtained. Subtracted images were obtained by subtracting unenhanced data from enhanced data of identical volumes, and maximum intensity projection images were reconstructed, which two observers independently evaluated in blinded fashion, with conventional angiographic results as the standard of reference.
\end{abstract}

Results: Sensitivity and specificity for grading of hemodynamically significant stenoses ( $250 \%$ lumen reduction) on subtracted MR angiograms were $94 \%$ and $93 \%$, respectively. Sensitivity of subtracted images was significantly higher compared with that of time-of-flight images $(p<.05)$ but not with that of nonsubtracted images. Contrast-to-noise ratio on subtracted images was significantly higher compared with that on nonsubtracted images $(\mathrm{p}<.05)$ but not with that on time-of-flight images. There was good correlation between stenosis length measurements on gadoliniumenhanced MR angiograms and those on conventional angiograms.

Conclusion: Subtracted MR angiography is superior to cardiac-synchronized timeof-flight MR angiography for imaging of iliac and upper femoral arteries and provides higher contrast-to-noise ratio, fewer artifacts, and easier image interpretability than nonsubtracted MR angiography. 
Chapter 4 


\section{Introduction}

Various magnetic resonance (MR) imaging techniques such as time-of-flight (TOF) MR angiography (1-7), phase-contrast MR angiography (8-9), gadolinium-enhanced MR angiography (10-15), or a combination of techniques (16-17) have been used for imaging of the iliac arteries in both patients and healthy volunteers (18-19).

Most of the more conventional techniques (eg, TOF MR angiography) suffer from artifacts and are time-consuming $(9,18)$. Use of cardiac synchronization can prevent some of these artifacts at TOF MR angiography. In non-cardiac-synchronized TOF MR angiography, image quality deteriorates because of large variations in flow velocities across the cardiac cycle, vessel pulsatility, and diastolic backflow of saturated blood from the presaturation slab in cases of vessels with a triphasic waveform. Despite the improvement achieved with cardiac synchronization, there still is room for improvement in terms of both imaging time and accuracy $(7,18)$.

Coronal volumes can be acquired with gadolinium-enhanced MR angiography. Gadolinium-induced T1 shortening prevents artifacts such as inplane saturation and flow voids caused by outflow effects and poststenotic turbulence (20). However, use of gadolinium also has disadvantages. At some time after injection, venous blood and surrounding tissue (muscle) will acquire shorter T1 values, as well. This can result in venous overprojection and lower vessel-to-background contrast. To prevent these effects, one can decrease the repetition and echo times $(11,21,22)$ so that the volume is imaged before venous return or tissue T1 decrease occurs. This technique is now possible by using MR imagers with fast gradient systems. However, as demonstrated in this study, venous return can also be postponed (sufficiently) with a low injection rate to allow gadolinium-enhanced MR angiography with a conventional MR imager.

The consequence of a low injection rate is lower vessel-to-background contrast due to lower intra-arterial gadolinium concentration. This decrease in contrast can be overcome by using a subtraction technique (13-14). Subtraction of a precontrast volume from a gadolinium-enhanced volume (volume acquired during continuous infusion of gadolinium) will lower background signal intensities and thus increase vessel-to-background contrast.

The purpose of this study was to compare the results of three-dimensional (3D) gadolinium-enhanced MR angiography (subtracted and nonsubtracted) with twodimensional systolic cardiac-synchronized TOF MR angiography, with conventional angiographic results as the standard of reference.

\section{Materials and Methods}

Twenty-eight consecutive patients, 21 men and seven women aged 32-84 years (mean, 63 years), were included in the study. The patients, who were scheduled for conventional angiography, had complaints of intermittent claudication and one or more stenotic lesions in the iliac or upper femoral arteries proved at Doppler ultrasound (US). The patients underwent MR angiography of the iliac and upper femoral arteries; 3D gadolinium-enhanced MR angiography with and without 
subtraction was performed in 28 patients, and two-dimensional systolic cardiacsynchronized TOF MR angiography was performed in 24 patients.

Approval was obtained from the medical ethics commission of the hospital, and written informed consent was obtained from all patients before examination. Conventional angiography was performed in all patients, the results of which served as the standard of reference.

\section{MR Angiography}

MR angiograms were obtained with a 1.5-T system (Gyroscan Advanced Clinical System-II, release 3.6; Philips Medical Systems, Best, The Netherlands) with a maximum gradient strength of $10 \mathrm{mT} / \mathrm{m}$ and a slew rate of $10 \mathrm{mT} / \mathrm{m} / \mathrm{msec}$. The body coil was used for signal transmission and reception.

The TOF technique was a sequentially ordered systolic cardiac-synchronized multiple two-dimensional magnetization-prepared gradient-recalled-echo (turbo "field-echo") technique (15/6.8 [repetition time msec/echo time msec], $50^{\circ}$ flip angle preceded by $180^{\circ}$ inversion prepulse). The sequence was divided into six turbo shots (inversion pulses) of 30 phase-encoding steps each. Six turbo shots were needed to image a single section. During each heartbeat, one turbo shot was acquired (six heartbeats for one section). The trigger delay for systolic cardiac synchronization was set to sample the middle of $\mathrm{K}$-space during maximum systolic flow $(280 \mathrm{msec}$ after Q-R-S-complex detection). A detailed diagram of this pulse sequence is shown in Figure 1.

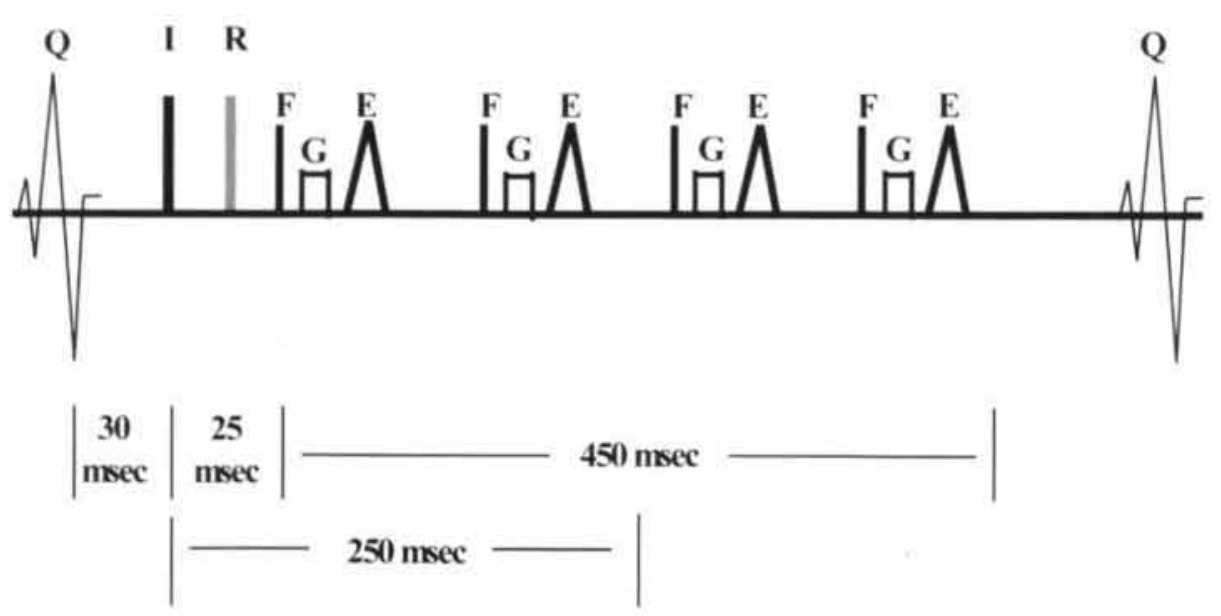

Figure 1. Diagram shows the timing sequence for systolic cardiac-synchronized TOF MR angiography. One heart cycle is shown in which one turbo shot is performed. At $30 \mathrm{msec}$ after detection of the Q-R-S complex (Q), the acquisition starts with application of the $180^{\circ}$ inversion prepulse (I). In the 25 -msec delay between the inversion prepulse and the first $50^{\circ}$ flip angle $(\mathrm{F})$, the venous presaturation prepulse (R), or rest pulse, is applied. The middle of $\mathrm{K}$-space $(\mathrm{K})$ is sampled $250 \mathrm{msec}$ after the $180^{\circ}$ inversion prepulse 
(middle of K-space sampling). The turbo shot lasts from $30 \mathrm{msec}$ to $505 \mathrm{msec}$ after detection of the Q-R-S complex. $\mathrm{E}=$ echo, $\mathrm{G}=$ gradient inversion.

The $180^{\circ}$ inversion prepulse, applied before each turbo shot, can nullify the signal from any desired tissue. In this study, the prepulse was used to nullify the signal from fat by applying it $250 \mathrm{msec}$ before sampling of the middle of $\mathrm{K}$-space $(7,18)$. A $250 \times 250-$ mm field of view and a $256 \times 180$ matrix resulted in an inplane resolution of 1.0x1.4-mm. Section thickness was $5-\mathrm{mm}$ with a $2-\mathrm{mm}$ overlap, and one signal was acquired. A 100-mm-thick tracking venous presaturation slab (rest slab) with an 8$\mathrm{mm}$ slab gap caudal to the imaged section was used to suppress venous signal. The sections were planned to cover the stenoses found at Doppler US. Eighty sections were thus obtained, which covered $240-\mathrm{mm}$ of the vascular tree. Acquisition of 80 sections, with a patient's heart rate of 80 beats per minute, took 8 minutes.

Maximum intensity projection (MIP) images were reconstructed in three orthogonal directions and were printed on film. These MIP images also served as scout images to plan the volume for gadolinium-enhanced MR angiography.

In the four patients who did not undergo systolic cardiac-synchronized TOF MR angiography, a TOF MR angiographic technique was used with parameters identical to those used with the cardiac-synchronized TOF technique (but without cardiac synchronization), and only 40 sections (10-mm section thickness) were acquired.

Before gadolinium-enhanced MR angiography, a timing sequence was performed to determine the arrival-time of the gadolinium bolus in the imaged volume. This gradient-recalled-echo technique consisted of dynamic (21.7/3.4) imaging of an axial section through the abdominal aorta 40 times, with a temporal resolution of 1.4 seconds. With this dynamic sequence, the preparation phase of the acquisition is performed only once; thus, all imaged sections are identical. A 100 -mm-thick caudal presaturation slab was used to suppress signal from inflowing venous blood, and an 80 -mm-thick cranial presaturation slab was used to prevent inflow effects from arterial blood. A test bolus of 1-mL of gadopentetate dimeglumine (Magnevist; Schering, Berlin, Germany) with a subsequent flush of $20-\mathrm{mL}$ of normal saline was injected with the same injection rate as that used for the gadolinium-enhanced MR angiographic technique $(0.3-\mathrm{mL} / \mathrm{sec})$. The dynamic sequence and the injection of 1 $\mathrm{mL}$ of gadopentetate dimeglumine (and subsequent $20-\mathrm{mL}$ normal saline flush) were started simultaneously. The images were then evaluated for an increase in signal intensity in the abdominal aorta, which represented the arrival of the contrast material bolus. The number of the section on which the elevated intra-aortal signal intensity was seen multiplied by the temporal resolution per section was the arrival-time of the gadolinium bolus in the imaged volume (ie, imaging delay time).

Gadolinium-enhanced MR angiography was performed with a 3D gradient-recalledecho (fast - field-echo $)$ ) technique $\left(20 / 6,9 ; 60^{\circ}\right.$ flip angle; $90 \%$ rectangular field of view). Twenty-five 3-mm-thick coronal sections (total volume thickness, $75-\mathrm{mm}$ ) were acquired overcontiguously (interpolation of the 253 -mm-thick sections to 50 1.5 -mm-thick sections) in 91 seconds with a $400 \times 360-\mathrm{mm}$ field of view and a $512 \times 205$ matrix, which resulted in a voxel volume of $4.5-\mathrm{mm}^{3}$. This technique was also implemented in a dynamic sequence in which two identical volumes were acquired: one before the administration of gadopentetate dimeglumine (mask image) and one during continuous infusion of the gadolinium-chelates (contrast-enhanced image). 
In three volunteers (each of whom underwent imaging on four separate occasions) and in three patients, the optimum dose of gadopentetate dimeglumine was determined by varying the dose for the 91-second study. By using a constant injection rate to obtain an even concentration of intravascular contrast material throughout the acquisition, we determined the lowest dose possible that still yielded high enough vessel-to-background contrast. Empirically, we determined that injection of $29-\mathrm{mL}$ of gadopentetate dimeglumine at a rate of approximately $0.3-\mathrm{mL} / \mathrm{sec}$ was sufficient to ensure high quality MR angiographic images. Thus, for the current study, 29-mL of preheated gadopentetate dimeglumine was administered by using an 18-gauge intravenous cannula (Venflon 2; Ohmeda, Helsingborg, Sweden) in an antecubital vein. The injection was started before the beginning of the acquisition, with an imaging delay determined on the basis of the timing sequence. Gadopentetate dimeglumine was administered over 91 seconds; thus, administration of the contrast agent stopped before the end of the acquisition. The contrast agent was injected by hand; a subsequent flush $(20-\mathrm{mL}$ of normal saline) ensured that all $29-\mathrm{mL}$ of gadopentetate dimeglumine was administered and flushed through the veins.

After the unenhanced and contrast-enhanced volumes were acquired, subtraction of the mask volume from the contrast-enhanced volume was performed, which resulted in a subtracted gadolinium-enhanced MR angiographic volume. MIP images were constructed from both nonsubtracted and subtracted contrast-enhanced volumes in three orthogonal directions and were printed on film.

\section{Conventional Angiography}

Conventional angiography was performed (Diagnostic Arc x-ray system; Philips Medical Systems) within 1 week of MR angiography. The angiography unit was equipped with a programmable stepping $\mathrm{C}$ arm, a film changer (Puck; Elema Schonander, Solina, Sweden), an add-on digital subtraction angiography system (Technicare DR 960-B; GE Medical Systems, Milwaukee, Wis), and a power injector (MEDRAD, Pittsburgh, Pa). All angiographic procedures were supervised by an experienced vascular radiologist (M.W.d.H.).

The standard protocol consisted of puncture of the common femoral artery and placement of a 5-F catheter in the distal aorta just above the bifurcation (if examination of two legs was necessary) or use of a crossover approach (if examination of only one leg was necessary). A nonionic contrast medium (iohexol, Omnipaque; Nycomed, Cork, Ireland) was used, with variable volumes and flow rates that depended on the location of the catheter tip and on the imaging system (digital subtraction or conventional angiography). Film hard copies were obtained in the posteroanterior direction, and, in most patients, additional posteroanterior and oblique views were obtained at digital subtraction angiography.

\section{Image Evaluation}

For evaluation purposes, the arterial tree on the MIP and conventional angiographic images was divided into the following segments: aorta, common iliac artery, external 
iliac artery, common femoral artery, superficial femoral artery, and deep femoral artery. Image quality was analyzed in two ways: (a) by objectively measuring signal intensities and calculating contrast-to-noise ratios (C/Ns) and (b) by comparing the validity of MR angiographic image interpretations of degree and length of stenoses, with conventional angiographic results as the standard of reference.

$\mathrm{C} / \mathrm{N}$ was defined as (mean signal intensity of the vessel - mean signal intensity of surrounding tissue)/standard deviation of noise. All evaluations and signal intensity measurements were performed with MIP images. To measure intra-arterial signal intensities, an operator-dependent region of interest was drawn in vessel segments that were evaluated as having no abnormality on conventional angiograms. For each vessel segment used for intravascular signal intensity measurement, a $10-\mathrm{cm}^{2}$ region of interest was drawn to cover surrounding tissue adjacent to the vessel segment. Because MIP images did not depict any air (noise), a $2-\mathrm{cm}^{2}$ region of interest was drawn in an area of homogeneous signal intensity (in fatty tissue of the groin region) to measure the standard deviation of the included signal intensities. On cardiacsynchronized TOF MIP angiograms, intravascular signal intensities could not be calculated for all separate segments of the arterial tree, because of flow voids due to intrastenotic outflow and poststenotic turbulence effects in some vascular segments.

All three MR angiographic techniques were evaluated independently by two observers who were unaware of the results of the other imaging techniques and of Doppler US. The observers, a vascular radiologist (M.W.d.H.) and a vascular surgeon (P.J.E.H.M.K.), evaluated all imaged vessel segments with regard to degree and length of stenoses. This was done by assessing all stenoses per vessel segment and categorizing them with a five-point scale $(1=$ no abnormality [ $0 \%$ to $20 \%$ stenosis], 2 $=20 \%$ to $50 \%$ stenosis, $3=50 \%$ to $75 \%$ stenosis, $4=75 \%$ to $100 \%$ stenosis, and $5=$ complete obstruction) $(9,20,23)$. On cardiac-synchronized TOF MR angiograms, some stenosis percentages could not be determined due to complete signal loss in and distal to the stenosis. In these cases, complete signal loss over a distance smaller than $10-\mathrm{mm}$ was graded as a $50 \%$ to $75 \%$ stenosis (score $=3$ ) and signal loss equal to or longer than $10-\mathrm{mm}$ and smaller than $30-\mathrm{mm}$ was graded as a $75 \%$ to $100 \%$ stenosis $($ score $=4)$. Complete obstruction on cardiac-synchronized TOF MR angiograms was defined as complete signal loss equal to or longer than $30-\mathrm{mm}$ (7). The length of stenoses on cardiac-synchronized TOF MR angiographic images was defined as the length of intravascular signal void and/or diminished signal intensity or, on gadolinium-enhanced MR angiograms, as the length of a vessel segment with an abnormal diameter compared with that of a normal segment. To maximize measurement accuracy, a ruler with $0.5-\mathrm{mm}$ markings was used.

Conventional angiographic images were evaluated with regard to degree and length of stenoses by the two observers and were categorized in the same way as the MR angiograms. This was done during separate sessions 4 weeks before the interpretations of the MR angiograms. The observers had to reach consensus about the conventional angiograms. If no consensus was reached, a third observer (J.M.A.v.E.) decided on the point of disagreement. The validity of the three MR angiographic techniques, in terms of detection and grading of stenoses and of stenosis length measurements, was determined by comparing the results with those of conventional angiography (the standard of reference). 


\section{Statistical Analysis}

Pairwise comparison of mean $\mathrm{C} / \mathrm{N}$ per vessel segment for the three techniques was accomplished by using the Wilcoxon signed-rank test, with two-tailed p-values for normal approximation. P-values of less than .05 were considered to indicate statistically significant differences. Interobserver agreement for grading of stenoses with the three techniques was determined by calculating the Pearson correlation coefficient and kappa statistic. The Pearson correlation coefficient is a measure of the association between two random variables, given that these variables have a linear relationship (24).

Linear weighted $\kappa$ values were calculated $(25-26)$. The $\kappa$ value can range from - 1 (no agreement) to 1 (perfect agreement). The $\kappa$ value can be interpreted as poor $(\kappa=$ 0 ), slight ( $\kappa$ of $0.0-0.2$ ), fair ( $\kappa$ of $0.21-0.40$ ), moderate ( $\kappa$ of $0.41-0.60)$, substantial $(\kappa$ of $0.61-0.80$ ), and almost perfect ( $\kappa$ of $0.81-1.00)(27)$.

Sensitivity and specificity of the three MR angiographic techniques were calculated for the presence of diseased vessel segments (reduction of lumen diameter $220 \%$ ) and for the presence of hemodynamically significant stenoses (reduction of lumen diameter $250 \%$ ), with conventional angiographic results as the standard of reference.

Because fewer vessel segments were depicted at cardiac-synchronized TOF MR angiography, sensitivity and specificity are not directly comparable with sensitivity and specificity of gadolinium-enhanced MR angiography. Therefore, we used the McNemar symmetry test to determine any statistically significant differences between sensitivity and specificity (Table 1 ).

Table 1. Sensitivity and Specificity of MR Angiography.

\begin{tabular}{|c|c|c|c|c|}
\hline \multirow[b]{2}{*}{ Sequence and Observer } & \multicolumn{2}{|c|}{$\begin{array}{l}\text { Lumen Reduction } \\
220 \%\end{array}$} & \multicolumn{2}{|c|}{$\begin{array}{l}\text { Lumen Reduction } \\
250 \%\end{array}$} \\
\hline & $\begin{array}{l}\text { Sensitivity } \\
(\%)\end{array}$ & $\begin{array}{l}\text { Specificity } \\
(\%)\end{array}$ & $\begin{array}{l}\text { Sensitivity } \\
(\%)\end{array}$ & $\begin{array}{l}\text { Specificity } \\
(\%)\end{array}$ \\
\hline \multicolumn{5}{|l|}{ TOF MR angiography* } \\
\hline Observer 1 & 77 & 87 & 72 & 91 \\
\hline Observer 2 & 75 & 90 & 81 & 93 \\
\hline \multicolumn{5}{|c|}{ Nonsubtracted MR angiography $\dagger$} \\
\hline Observer 1 & $87 \ddagger$ & 88 & 90 & 89 \\
\hline Observer 2 & 87 & 89 & 95 & 92 \\
\hline \multicolumn{5}{|c|}{ Subtracted MR angiography $\dagger$} \\
\hline Observer 1 & $89 \pm$ & 87 & $92 \ddagger$ & 93 \\
\hline Observer 2 & $90 \pm$ & 90 & $95 \ddagger$ & 93 \\
\hline
\end{tabular}

- Data are based on findings in 24 patients. $\uparrow$ Data are based on findings in 28 patients. $\ddagger$ Difference from result at TOF MR angiography was statistically significant $(p<.05)$.

With this test, the results used to calculate sensitivity and specificity are compared, and only those results are included that are present for both observers. A p-value of less than .05 was considered to indicate a statistically significant difference. The relationship between MR angiographic and conventional angiographic scores as regards degree and length of stenosis was analyzed by using standard linearregression analysis (28).

The Pearson correlation coefficient and orthogonal regression analysis were used, to assess interobserver agreement with regard to length of stenoses. Orthogonal 
regression analysis can be used to determine the regression analysis for two independent variables (eg, observers). The relationship between the two observers in terms of length measurements as calculated with the orthogonal regression analysis is given by the following equation: $\left(o b s_{1}\right)=\alpha\left(o b s_{2}\right)+\beta$, where obs $s_{1}$ is the length (in millimeters) measured by observer 1 , obs $2_{2}$ is the length measured by observer $2, \alpha$ is slope of the regression line (ideally, $\alpha=1$ ), and $\beta$ is the intercept of the regression line (ideally, $\beta=0$ ).

\section{Results}

All patients were able to tolerate MR angiography. Of the 28 patients examined with MR angiography, four were not examined with cardiac-synchronized TOF MR angiography due to time limitations (in three patients) and inadequate cardiac triggering signal (in one patient with cardiac arrhythmia). Instead, only TOF MR angiograms without cardiac synchronization were obtained as scout images for gadolinium-enhanced MR angiography.

Table 2 lists the location, number, and degree of stenotic lesions as demonstrated at conventional angiography.

Table 2. Stenoses Demonstrated at Conventional Angiography in 28 Patients.

\begin{tabular}{|c|c|c|c|c|c|}
\hline \multirow[b]{2}{*}{ Artery } & \multicolumn{5}{|c|}{ Stenosis Grade } \\
\hline & 1 & 2 & 3 & 4 & 5 \\
\hline Aorta & 23 & 0 & 0 & 0 & 0 \\
\hline Common iliac & 22 & 9 & 7 & 3 & 4 \\
\hline External iliac & 33 & 4 & 8 & 0 & 1 \\
\hline Common femoral & 42 & 0 & 3 & 1 & 0 \\
\hline Superficial femoral & 34 & 1 & 3 & 0 & 3 \\
\hline Deep femoral & 37 & 0 & 2 & 0 & 2 \\
\hline
\end{tabular}

Note.--Data are number of stenotic lesions.

A conventional angiographic image and the corresponding representative examples of the three MR angiographic techniques are shown in Figure 2.

A

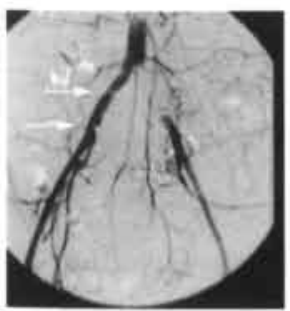

B

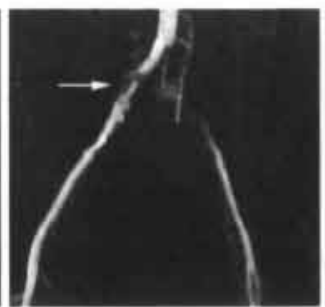

C

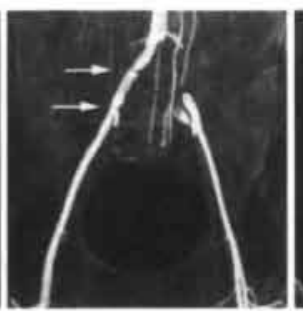

D

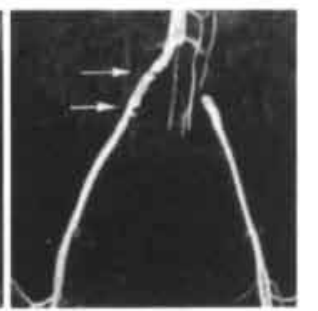

Figure 2. Images obtained in a 52-year-old man. A: Conventional angiogram of the iliac and upper femoral arteries shows two severe stenoses (arrows) of the right common iliac artery and complete obstruction of the left common iliac artery. B: Cardiac-synchronized TOF MR angiographic MIP image shows good vessel-to-background contrast but diminished intrastenotic signal intensity in right common iliac artery (arrow) and flow voids distal to the obstruction in the left common iliac artery. C: Nonsubtracted gadolinium-enhanced MR angiographic MIP image depicts the two stenoses (arrows) in the 
right common iliac artery and complete obstruction in the left common iliac artery, without the flow voids seen on the image in B. D: Subtracted gadolinium-enhanced MR angiographic MIP image shows better vessel-to-background contrast compared with the image in $\mathrm{C}$, owing to decreased background signal intensities and fewer artifacts.

Both observers judged image quality of all MR angiographic images to be high enough for evaluation purposes.

After evaluation of all three MR angiographic techniques, the observers concluded that subtracted gadolinium-enhanced MR angiograms had the best overall image quality and the fewest artifacts. The $\mathrm{C} / \mathrm{N}$ per vessel per segment per technique (Table 3) confirmed this subjective opinion.

Table 3. Mean Contrast-to-noise (C/Ns) on MR Angiograms.

\begin{tabular}{llll} 
Artery & $\begin{array}{l}\text { TOF MR } \\
\text { Angiography }\end{array}$ & $\begin{array}{l}\text { Nonsubtracted } \\
\text { Angiography* }\end{array}$ & $\begin{array}{l}\text { MR Subtracted MR } \\
\text { Angiography }\end{array}$ \\
\hline Aorta & $45.0 \dagger$ & 34.4 & 40.2 \\
Common iliac & $48.5 \dagger$ & 34.3 & 41.2 \\
External iliac & $49.4 \ddagger$ & 40.1 & 48.8 \\
Common femoral & $46.0 \ddagger$ & 37.9 & 48.1 \\
Superficial femoral & $47.8 \ddagger$ & 33.3 & 45.1 \\
Deep femoral & $42.4 \ddagger$ & 33.6 & 44.1
\end{tabular}

* $\mathrm{C} / \mathrm{N}$ was statistically significantly different from that obtained on subtracted MR angiograms $(\mathrm{p}<.05)$. $\dagger$ $\mathrm{C} / \mathrm{N}$ was statistically significantly different from those obtained on nonsubtracted $(\mathrm{p}<.05)$ and subtracted $(\mathrm{p}<.05) \mathrm{MR}$ angiograms. $\ddagger \mathrm{C} / \mathrm{N}$ was statistically significantly different from that obtained on nonsubtracted MR angiograms $(p<.05)$.

In all vessel segments, subtracted gadolinium-enhanced MR angiograms had statistically significantly higher $\mathrm{C} / \mathrm{Ns}$ than nonsubtracted gadolinium-enhanced MR angiograms. The only statistically significant differences in $\mathrm{C} / \mathrm{N}$ between subtracted gadolinium-enhanced MR angiograms and systolic cardiac-synchronized MR angiograms were in the aorta and common iliac arteries. Compared with nonsubtracted gadolinium-enhanced MR angiograms, cardiac-synchronized TOF MR angiograms had statistically significant higher $\mathrm{C} / \mathrm{Ns}$ in all vessel segments except the deep femoral artery.

Interobserver agreement ( $\kappa$ values and Pearson correlation coefficients) among the two observers about the degree of stenoses demonstrated with each technique are listed in Table 4.

Table 4. Interobserver Variability at MR Angiography.

\begin{tabular}{llll}
$\begin{array}{l}\text { MR Angiographic } \\
\text { Sequence }\end{array}$ & $\kappa$ Value* & $\begin{array}{l}95 \% \text { Confidence } \\
\text { Interval }\end{array}$ & r value \\
\hline TOF & $0.72(0.04)$ & $0.63,0.80$ & .83 \\
Nonsubtracted & $0.73(0.03)$ & $0.67,0.80$ & .85 \\
Subtracted & $0.78(0.03)$ & $0.71,0.84$ & .86
\end{tabular}

- Numbers in parentheses are standard deviations.

The $\kappa$ values were calculated for all vessel segments and stenoses seen on MR angiographic MIP images. Because some vessel segments evaluated on the MIP images contained more than one stenosis, the total number of scores used for 
calculating $\kappa$ values exceeds the number of vessel segments evaluated on the conventional angiographic images listed in Table 2. For the cardiac-synchronized TOF technique, 225 scores were used. For the nonsubtracted and subtracted gadolinium-enhanced MR angiographic techniques, 302 and 304 scores were used, respectively.

Table 1 lists the sensitivity and specificity for detection and grading of diseased vessel segments (lumen diameter reduction $220 \%$ ) and of hemodynamically significant stenoses (lumen diameter reduction $250 \%$ ).

In total, 10 completely obstructed vessel segments were found at conventional angiography. Eight obstructions were classified as such by both observers with each MR angiographic technique. Both observers overlooked one complete obstruction on both the subtracted and the nonsubtracted gadolinium-enhanced MR angiographic images and classified this lesion as not obstructed. Another complete obstruction was overlooked by both observers on the cardiac-synchronized TOF images, owing to complete flow void in that vessel, and by one observer on the subtracted gadoliniumenhanced MR angiographic image, owing to misinterpretation of vessel anatomy (the deep femoral artery was thought to be the common femoral artery; therefore, complete obstruction of the superficial femoral artery was overlooked). Figure 3 shows the results of the linear-regression analysis, in which correlation between the degree of a stenosis on MR angiograms and that on conventional angiograms is shown.

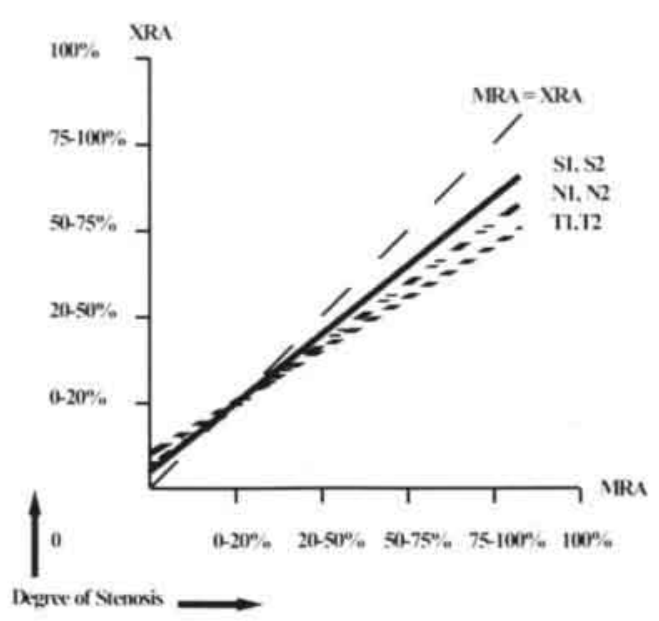

Figure 3. Graph shows linearregression analysis results of both observers for degree of stenosis determined with subtracted (S), nonsubtracted $(\mathrm{N})$, and TOF $(\mathrm{T}) \mathrm{MR}$ angiograms (MRA) and conventional angiograms (XRA). Note the tendency to overestimate degree of stenosis with all MR angiographic techniques relative to conventional angiography. The dashed line that intersects the $y$ axis at 0 indicates ideal results, in which MR angiographic results predict conventional angiographic results in all cases $(M R A=X R A)$.

Comparisons of the length of stenoses measured on images obtained with the MR angiographic techniques were performed, with conventional angiographic measurements as the standard of reference. On conventional angiograms, hemodynamically significant stenoses were 1-50-mm long. On cardiac-synchronized TOF angiograms, the mean difference in length measurements between observers was 7.6- $\mathrm{mm} \pm 8.8$ (1 standard deviation); on nonsubtracted gadolinium-enhanced $\mathrm{MR}$ 
angiograms, the mean difference was $5.3-\mathrm{mm} \pm 5.3$; and on subtracted gadoliniumenhanced MR angiograms, the mean difference between was $5.1-\mathrm{mm} \pm 5.6$.

The Pearson correlation coefficient ( $\mathrm{r}$ ) between the two observers per MR angiographic technique for the length measurements of hemodynamically significant stenoses was .77 for cardiac-synchronized TOF MR angiography, .74 for nonsubtracted gadolinium-enhanced MR angiography, and .70 for subtracted gadolinium-enhanced MR angiography. The relationships between length measurements of the two observers, calculated with orthogonal regression analysis, are described by the following equations: for the cardiac-synchronized TOF technique, $($ measurements by observer 1$)=1.1 \times$ (measurements of observer 2$)+1.0$ $\mathrm{mm}$; for the nonsubtracted gadolinium-enhanced technique, (measurements by observer 1$)=1.3 \times$ (measurements by observer 2$)+2.3-\mathrm{mm}$; and for the subtracted gadolinium-enhanced technique, (measurements by observer 1) $=1.0 \mathrm{x}$ (measurements by observer 2 ) +4.6 -mm. Figure 4 shows the correlation, based on linear-regression analysis results, between stenosis length measurements on conventional angiograms and those on MR angiograms.

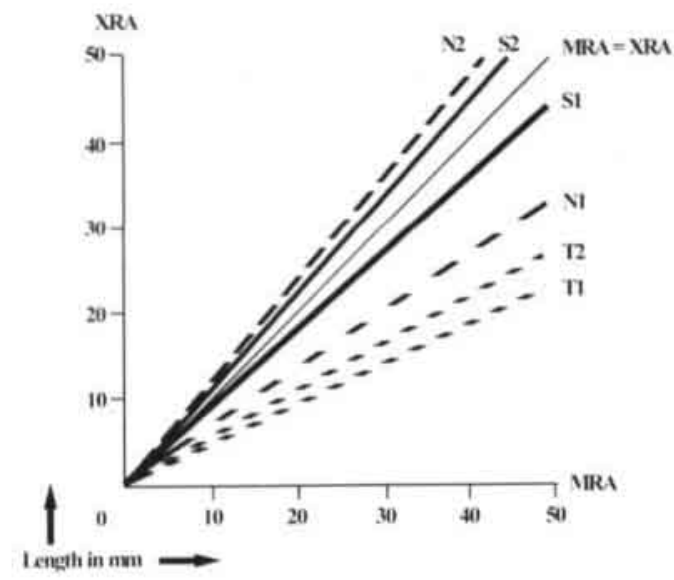

Figure 4. Graph shows linearregression analysis results for determination of length of hemodynamically significant stenosis with all three MR angiographic (MRA) techniques. Note that the scores of both gadolinium-enhanced MR angiographic techniques are either slight over- or underestimates of stenosis length (determined at conventional angiography [XRA]). The dotted line that intercepts the $\mathrm{y}$ axis at 0 indicates ideal results in which MR angiographic results predict conventional angiographic results in all cases $(\mathrm{MRA}=\mathrm{XRA}) . \mathrm{NI}=$ results of observer 1 with nonsubtracted images, $\mathrm{N} 2=$

results of observer 2 with nonsubtracted images, S1 results of observer 1 with subtracted images, S2 results of observer 2 with subtracted images, $\mathrm{T} 1$ = results of observer 1 with TOF images, $T 2$ = results of observer 2 with TOF images.

\section{Discussion}

The goal of our study was to compare nonsubtracted and subtracted 3D gadoliniumenhanced MR angiography with two-dimensional systolic cardiac-synchronized TOF MR angiography, with conventional angiography as the standard of reference. We demonstrated that subtracted MR angiography had statistically significantly higher sensitivity than cardiac-synchronized TOF MR angiography (for both detection and grading of diseased vessel segments and hemodynamically significant stenoses) and statistically significantly higher $\mathrm{C} / \mathrm{Ns}$ than nonsubtracted MR angiography. The only 
statistically significant $\mathrm{C} / \mathrm{N}$ differences between subtracted $\mathrm{MR}$ angiography and cardiac-synchronized TOF MR angiography were for images of the aorta and common iliac arteries, where higher $\mathrm{C} / \mathrm{N}$ s were found with cardiac-synchronized TOF images. The lower $\mathrm{C} / \mathrm{Ns}$ found on nonsubtracted and subtracted MR angiograms is probably the result of bowel and respiratory motion due to the relatively long $3 \mathrm{D}$ acquisition.

Although $\mathrm{C} / \mathrm{Ns}$ were comparable among the three techniques, they are probably not comparable with those of other investigators. Because our images did not contain any pure noise (eg, from air), we used an area with homogeneous signal intensities in the fatty tissue of the groin region on our MIP images. It is most likely that this "standard deviation of homogeneous signal intensities" is higher than the real "standard deviation of noise", because fat is known to be less homogeneous than air.

The TOF technique we used was a prepulsed cardiac-synchronized turbo "field echo" technique. This technique has known superiority over noncardiac-synchronized techniques and results in better image quality compared with non-prepulsed TOF MR angiography $(7,18)$. The drawback of cardiac-synchronization is the increased imaging time and more severe overestimation of the degree, length, and number of stenoses. To reduce imaging time, a trade-off was chosen between a reasonable acquisition time (8-12 minutes, depending on patient's heart rate) and imaging during systole (55-505 msec after detection of the Q-R-S complex), while the middle of Kspace was filled during peak systolic flow $(280 \mathrm{msec}$ after detection of the Q-Rcomplex).

Although the degree, length, and number of stenoses are more often overestimated when cardiac synchronization is used, image quality is consistently high. In the present study, the validity of cardiac-synchronized TOF MR angiographic results is improved relative to that of our previous study (7), in which we found the sensitivity and specificity for detection and grading of hemodynamically significant stenoses to be $68 \%$ and $90 \%$, respectively. This improvement may be due to a learning effect, because the observers, technique, and patient selection criteria used in the present study were the same as those used in the previous study.

To reduce imaging time and further improve accuracy as regards degree and length of stenoses, we started using 3D gadolinium-enhanced MR angiography and combined this method with a subtraction technique to increase vessel-to-background contrast, as proposed by Adamis et al (13). A subtraction technique not only increases $\mathrm{C} / \mathrm{N}$ significantly, but artifacts such as those due to inflow of venous nonsaturated blood into the imaged volume (Fig 5), wraparound artifact, and high signal intensity of bladder and ureter (Fig 6) are eliminated automatically. High signal intensity of bladder and ureter occurred owing to the 1-mL test bolus of gadopentetate dimeglumine used with the timing sequence and the relatively long imaging time for the gadolinium-enhanced technique. We started using this test bolus after it became apparent that the arrival-time of the bolus can vary substantially between patients (Johansson L.M.O., personal communication, August, 1996).

We compared all three MR angiographic techniques with conventional angiography as regards the grade of stenoses for diseased vessel segments (lumen diameter reduction $220 \%$ ) and hemodynamically significant stenoses (lumen diameter reduction $250 \%$ ). Although a $20 \%$ to $50 \%$ reduction in lumen diameter has no 
A

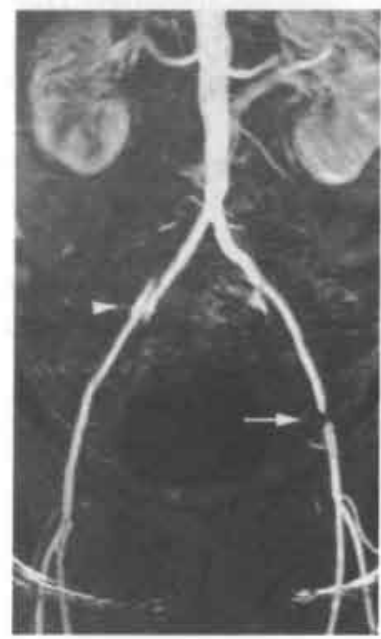

A

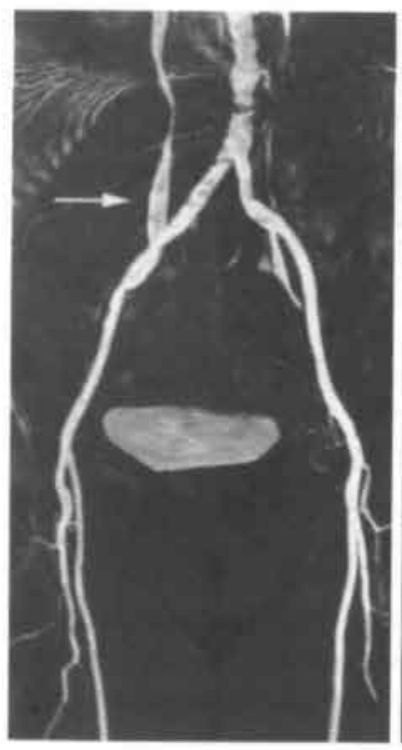

B

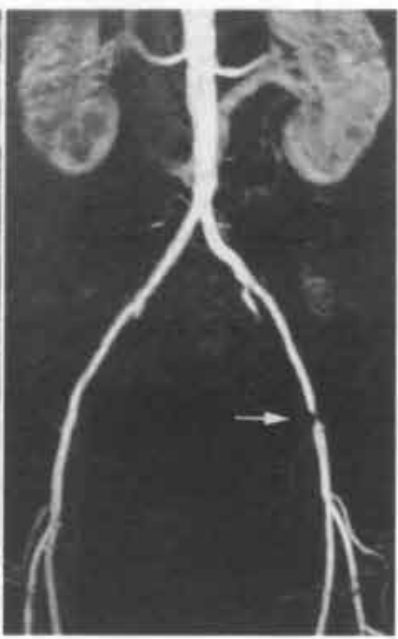

Figure 5. Gadolinium-enhanced MR angiographic MIP images in a 60-year-old man demonstrate the effect of venous nonsaturated blood flowing into the scanned volume. A: Nonsubtracted MR angiographic MIP image shows high-signal-intensity inflowing venous nonsaturated blood (arrowhead) lateral to the right external iliac artery and a severe stenosis (arrow) in the left common femoral artery, depicted as a complete obstruction. B: Subtracted MR angiographic MIP image shows elimination of the signal from inflowing venous blood lateral to the right external iliac artery and accurate depiction of the severe stenosis (arrow) in the left common femoral artery.

B

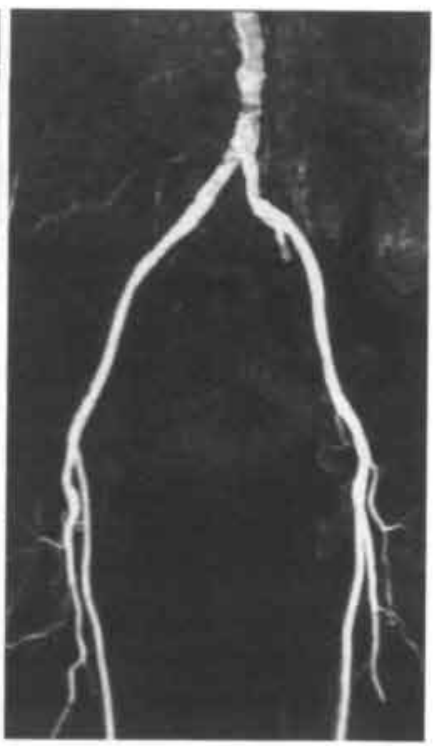

Figure 6. Gadoliniumenhanced MR angiographic MIP image in a 69-year-old woman shows elimination of artifacts in the subtracted MIP image. A: Nonsubtracted MR angiographic MIP image shows wraparound artifact from the patient's arms and high signal intensity in bladder and ureter (arrow). B: Subtracted MR angiographic MIP image shows fewer artifacts compared with the image in A.

clinical importance, inclusion of detection and grading results for these stenoses does give a better impression of the comparison between MR angiography and conventional angiography over the whole spectrum of pathologic conditions found in the arteries. The linear-regression analysis results depicted in Figure 3 show that degree of stenosis tends to be overestimated with all MR angiographic techniques. These results also demonstrate that the overestimations tend to be much smaller with 
both gadolinium-enhanced techniques compared with cardiac-synchronized TOF MR angiography. Overestimations that contribute to the fit of the graph in Figure 3 may also be caused by false-positive MR angiograms. Figure 7 shows a stenosis, which was best seen on the axial MIP image, that both observers overlooked on the conventional angiogram.

Gadolinium-enhanced MR imaging depicted complete obstructions remarkably well (Fig 8 ) owing to the relatively long infusion time, which allowed runoff vessels to be filled with contrast material during the acquisition.

A

B

C
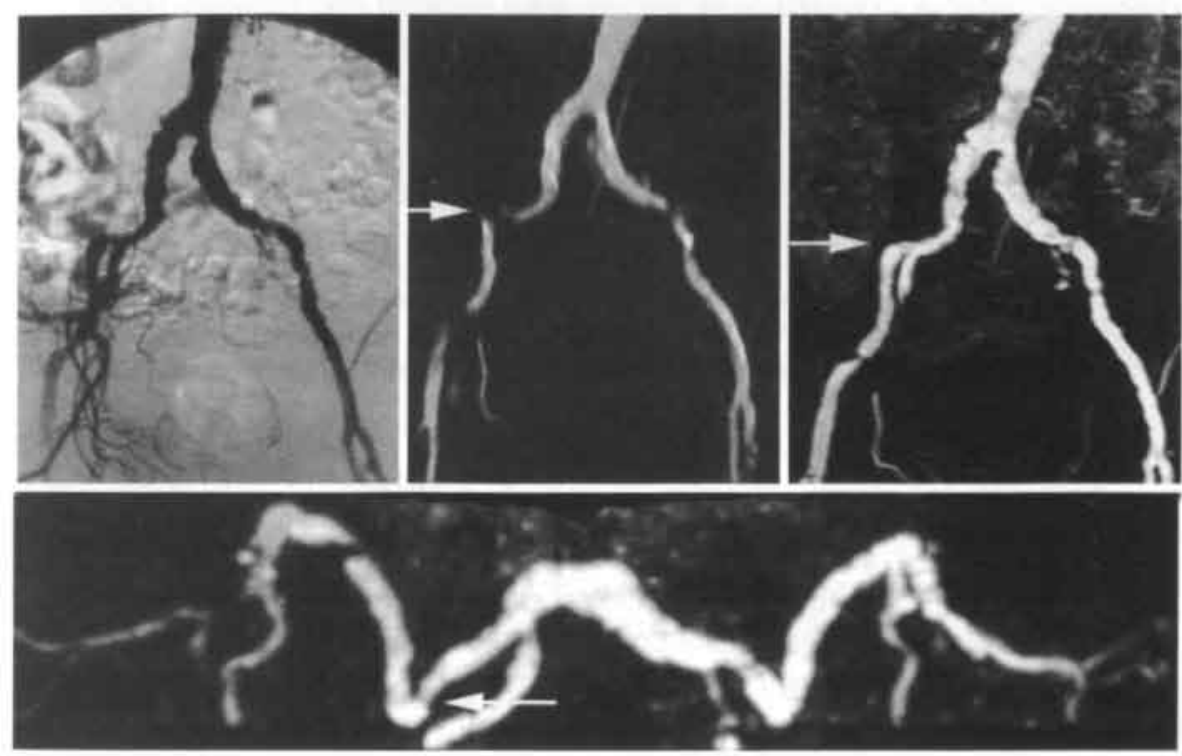

D

Figure 7. Image in a 77-year-old man. A: Conventional angiogram shows no clear sign of a stenosis at the junction of the right common and external iliac arteries (arrow). B: TOF MR angiographic MIP image shows flow void (arrow) at the junction of the right common and external iliac arteries, which suggests the presence of a stenosis or inplane saturation because of inplane flow. C: Coronal subtracted gadoliniumenhanced MR angiographic MIP image shows a difficult-to-appreciate stenosis (arrow) at the junction of the right common and external iliac arteries. D: The axial MIP image more clearly depicts this concealed stenosis (arrow). \{Three-dimensional views of the pelvic arteries can be seen on the Image-CD \}.

As expected, cardiac-synchronized TOF MR angiography could demonstrate runoff vessels, as well, but often was unable to demonstrate the exact length of complete obstructions. In one patient, one observer misinterpreted vessel anatomy on the subtracted MIP image. However, because nonsubtracted data were always available (ie, half the information used for the subtracted technique) in cases in which anatomic information is difficult to interpret, nonsubtracted MIP images can help resolve these problems.

The graph of the linear-regression analysis for length measurements (Fig 4) shows that both observers overestimated stenosis length on cardiac-synchronized TOF 
images. However, use of both types of gadolinium-enhanced MR angiographic images resulted in slight over- or underestimation of the length. Strecker et al

A

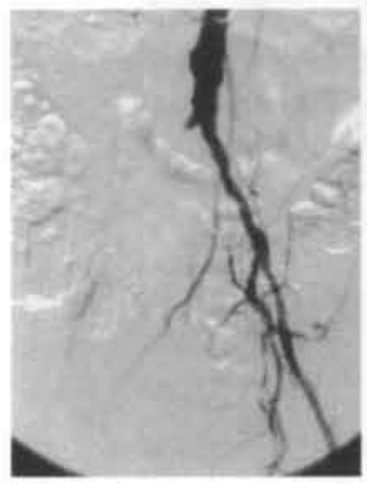

B

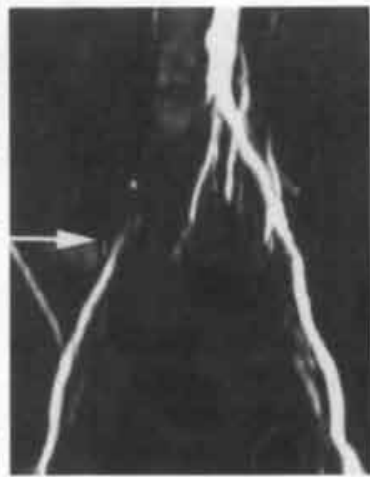

C

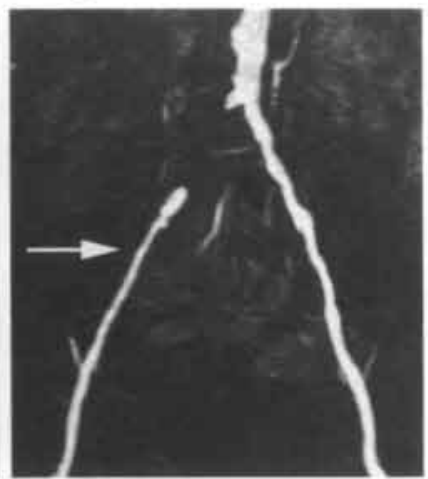

Figure 8. Images in a 56-year-old man illustrates differences between techniques in demonstration of runoff after complete obstruction. A: Conventional angiogram shows no clear runoff after complete obstruction of the right common iliac artery (arrow). B: TOF MR angiographic MIP image shows runoff (arrow) after complete obstruction of the right common iliac artery, with marked flow voids directly beyond the obstruction. C: Subtracted gadolinium-enhanced MR angiographic MP image shows the exact length of the complete obstruction plus the runoff of the vessel (arrow).

demonstrated that the length of stenoses and complete obstructions is clinically important if it exceeds $70-\mathrm{mm}$ (29). For lesions shorter than $70-\mathrm{mm}$, balloon angioplasty is indicated, and for those longer than 70-mm surgical intervention results in the best curative prognosis. In our study, no stenosis or complete obstruction was longer than $50-\mathrm{mm}$. Both types of gadolinium-enhanced MR angiographic techniques were associated with such small interpretation errors that these errors will have no therapeutic consequences for cases of stenosis or obstruction of the lengths found in our study.

All patients with symptom-producing arteriosclerotic peripheral vessel disease need an extensive diagnostic work-up, including noninvasive tests, to establish an accurate diagnosis. Precise anatomic knowledge of the complete peripheral vascular system (outflow study) is mandatory for treatment planning and to decide what type of intervention is most appropriate. A limitation of gadolinium-enhanced MR angiography, as used in this study, is the fact that only part of the information of a complete outflow study is acquired. Our results demonstrate that TOF MR angiography of the iliac and upper femoral arteries is best replaced with subtracted gadolinium-enhanced MR angiography to ensure statistically significantly better detection and grading of stenoses and to reduce imaging time. As regards the rest of the peripheral arteries, TOF MR angiography is probably still the best choice.

In conclusion, subtracted gadolinium-enhanced MR angiography is superior to cardiac-synchronized TOF MR angiography for imaging of the iliac and upper femoral arteries. The advantage of subtracted gadolinium-enhanced MR angiography over nonsubtracted gadolinium-enhanced MR angiography is statistically significantly increased $\mathrm{C} / \mathrm{N}$, fewer artifacts, and greater ease of image interpretation. 


\section{References}

1. Yucel EK, Kaufman JA, Geller SC, Waltman AC. Atherosclerotic occlusive disease of the lower extremity: prospective evaluation with two-dimensional time-of-flight MR angiography. Radiology 1993; 187:637-641.

2. Mulligan SA, Doyle M, Matsuda T, et al. Aortoiliac disease: two-dimensional inflow MR angiography with lipid suppression. JMRI 1993; 3:829-834.

3. Ecklund K, Hartnell GG, Hughes LA, Stokes KR, Finn JP. MR angiography as the sole method in evaluating abdominal aortic aneurysms: correlation with conventional techniques and surgery. Radiology 1994; 192:345-350.

4. Laissy JP, Limot O, Henry-Feugeas MC, et al. Iliac artery patency before and immediately after percutaneous transluminal angioplasty: assessment with time-offlight MR angiography.Radiology 1995; 197:455-459.

5. Snidow JJ, Harris VJ, Trerotola SO, et al. interpretations and treatment decisions based on MR angiography versus conventional arteriography in symptomatic lower extremity ischemia. JVIR 1995; 6:595-603.

6. Carpenter JP, Owen RS, Holland GA, et al. Magnetic resonance angiography of the aorta, iliac, and femoral arteries. Surgery 1994; 116: 17-23.

7. Ho KY, de Haan MW, Oei TK, et al. MR Angiography of the iliac and upper femoral arteries using four different inflow techniques. AJR 1997; 169:45-53.

8. Swan JS, Grist TM, Weber DM, Sproat IA, Wojtowycz MM. MR angiography of the pelvis with variable velocity encoding and a phased-array coil. Radiology 1994; 190:363-369.

9. Krug B, Kugel H, Harnischmacher U, et al. Diagnostic performance of digital subtraction angiography (DSA) and magnetic resonance angiography (MRA): preliminary results in vascular occlusive disease of the abdominal and lowerextremity arteries. Eurj Radiol 1995; 19:77-85.

10. Sivananthan UM, Ridgway JP, Bann K, et al. Fast magnetic resonance angiography using turbo-FLASH sequences in advanced aortoiliac disease. $\mathrm{Br}$ j Radiol 1993; 66:11031110.

11. Prince MR, Narasimham DL, Stanley JC, et al. Breath-hold gadolinium-enhanced MR angiography of the abdominal aorta and its major branches. Radiology 1995; 197:785-792.

12. Prince MR, Narasimham DL, Stanley JC, et al. Gadolinium-enhanced magnetic resonance angiography of abdominal aortic aneurysms. J Vasc Surg 1995; 21:656669.

13. Adamis MK, Li W, Wielopolski PA, et al. Dynamic contrast-enhanced subtraction MR angiography of the lower extremities: initial evaluation with a multisection twodimensional time-of-flight sequence. Radiology 1995; 196:689-695.

14. Douek PC, Revel D, Chazel S, Falise B, Villard J, Amiel M. Fast MR angiography of the aortoiliac arteries and arteries of the lower extremity: value of bolus-enhanced, whole-volume subtraction technique. AJR 1995; 165: 431-437.

15. Snidow JJ, Johnson MS, Harris VJ, et al. Three-dimensional gadolinium-enhanced MR angiography for aortoiliac inflow assessment plus renal artery screening in a single breath hold. Radiology 1996; 198:725-732.

16. Kaufman JA, Geller SC, Petersen MJ, Cambria RP, Prince MR, Waltman AC. MR imaging (including MR angiography) of abdominal aortic aneurysms: comparison with conventional angiography. AJR 1994; 163:203-210.

17. Snidow JJ, Aisen AM, Harris VJ, et al. Iliac artery MR angiography: comparison of three-dimensional gadolinium-enhanced and two-dimensional time-of-flight techniques. Radiology 1995; 196:371-378. 
18. Yucel EK, Silver MS, Carter AP. MR angiography of normal pelvic arteries: comparison of signal intensity and contrast-to-noise ratio for three different inflow techniques. AJR 1994; 163:197-201.

19. Yoshikawa K, Sugimura K, Kawamitsu H, Ishida T. Intrapelvic two-dimensional time-of-flight magnetic resonance angiography in healthy and diseased subjects. $\mathrm{Br} \mathrm{j}$ Radiol 1994; 67:140-146.

20. Furst G, Hofer M, Sitzer M, Kahn T, Muller E, Modder U. Factors influencing flowinduced signal loss in MR angiography: an in vitro study. J Comput Assist Tomogr 1995; 19:692- 699 .

21. Hendrick RE, Haacke EM. Basic physics of MR contrast agents and maximization of image contrast. JMRI 1993; 3:13 7-148.

22. Holland GA, Dougherty L, Carpenter JP, et al. Breath-hold ultrafast threedimensional gadolinium-enhanced MR angiography of the aorta and the renal and other visceral abdominal arteries. AJR 1996; 166:971-981.

23. Walstra BRJ. Arteriography of the lower leg: a comparative study between conventional arteriography and intra-arterial digital subtraction arteriography. Maastricht, The Nether lands: Datawyse Universitaire Pers Maastricht, 1993;90-95.

24. Kleinbaum DG, Kupper LL, Muller KE. Applied regression analysis and other multivariable methods. 2nd ed. Boston, Mass: PWS-Kent, 1988; 80.

25. Cohen J. A coefficient of agreement for nominal scales. Educ Psychol Meas 1960; 20:37-46.

26. Cohen J. Weighted kappa: nominal scale agreement with provision for scaled disagreement or partial credit. Psychol Bull 1968; 70:213-230.

27. Landis JR, Koch GG. The measurement of observer agreement for categorical data. Biometrics 1977; 33:159-174.

28. Hertz SM, Baum RA, Owen RS, Holland GA, Logan DR, Carpenter JP. Comparison of magnetic resonance angiography and contrast arteriography in peripheral arterial stenosis. Amj Surg 1993; 166:112-116.

29. Strecker EP, Boos IBL, Hagen B. Flexible tantalum stents for treatment of iliac artery lesions: long-term patency, complication, and risk factors. Radiology 1996; 199:641-647. 
Chapter 4

68 


\title{
Chapter 5
}

\section{Peripheral Vascular Tree Stenoses: Evaluation with Moving-Bed Infusion-tracking MR Angiography}

\author{
Published in Radiology 1998; 206:683-692
}

Authors: Ho K.Y.J.A.M., Leiner T., de Haan M.W., Kessels A.G.H., Kitslaar

P.J.E.H.M., van Engelshoven J.M.A. 



\section{Abstract}

Purpose: To evaluate a magnetic resonance (MR) angiographic technique for imaging of the peripheral arteries with gadolinium enhancement.

Materials and Methods: Moving-bed infusion-tracking MR angiograms were obtained in 15 healthy volunteers and in 28 patients with intermittent claudication before and during slow infusion of contrast material. Lower and upper leg and pelvic regions were imaged. Unenhanced images were subtracted from gadoliniumenhanced images, and maximum intensity projection images were generated. Image quality was evaluated subjectively and objectively, and maximum intensity projection images were compared with conventional angiograms, which served as the standard of reference.

Results: Moving-bed infusion-tracking MR angiography proved to be a robust technique, and image quality on maximum intensity projection images was comparable with that on conventional angiograms. Sensitivity and specificity for grading hemodynamically significant stenoses were $93 \%$ and $98 \%$, respectively, with excellent interobserver agreement.

Conclusion: Moving-bed infusion-tracking MR angiography can be used to image all peripheral arteries in 4 minutes by using a small amount of contrast material and a conventional 1.5-T MR imager. 
Chapter 5 


\section{Introduction}

Magnetic resonance (MR) angiography with gadopentetate dimeglumine enhancement has proved to be an important arterial imaging modality (1). Most techniques, however, allow imaging of only one coronal volume that covers $40-50 \mathrm{~cm}$ of the vascular tree. Although high image quality can be achieved with gadoliniumenhanced MR angiography, in some cases more than one field of view $(40-50 \mathrm{~cm})$ must be imaged. To accomplish imaging of the peripheral vascular tree, time-of-flight (TOF) techniques have been used (2). However, TOF imaging is time-consuming and subject to many artifacts (3).

An alternative to the TOF approach is imaging of multiple gadolinium-enhanced volumes with subsequent multiple doses of gadopentetate dimeglumine. Use of this method, however, increases the cost of the contrast material and can result in unintended effects due to an increase in intra- and extravascular levels of gadolinium. With the technique of moving-bed infusion-tracking MR angiography, only one bolus of gadopentetate dimeglumine $(39-\mathrm{mL})$ is used for imaging of the entire peripheral vascular tree, and the actual acquisition time is only 4 minutes.

The purpose of this study was to evaluate the feasibility of moving-bed infusiontracking MR angiography.

\section{Materials and Methods}

\section{MR Angiography}

All MR angiograms were obtained with a conventional 1.5-T MR system (Gyroscan Advanced Clinical System-II; Philips Medical Systems, Best, The Netherlands) with a gradient strength of $10 \mathrm{mT} / \mathrm{m}$ and a slew rate of $10 \mathrm{mT} / \mathrm{m} / \mathrm{msec}$. The body coil was used for signal transmission and reception for all studies. The procedure consisted of four parts: (a) positioning of the subject, (b) determination of arrival-time of gadopentetate dimeglumine with the use of a timing sequence, (c) determination of vessel anatomy with the use of four fast TOF sequences, and (d) moving-bed infusion-tracking MR angiography.

\section{Positioning of the Subject}

For subject positioning, the feet were placed in a holder, and a sandbag was placed over the feet to stabilize them. Two 30 -mm-wide bands were strapped around the lower legs and the two sandbags that had been placed on the sides of the legs, and one 30 -mm-wide band was strapped around the upper legs, to prevent patient movement. A fourth 200 -mm-wide band was strapped tightly around the thighs to minimize wraparound artifacts. The knees were elevated so the backs of the knees would be in the same horizontal plane as the heels of the feet. During infusion of the contrast 
agent, the subjects had to elevate their arms over their head, again to minimize wraparound artifacts. Figure 1 demonstrates subject positioning.

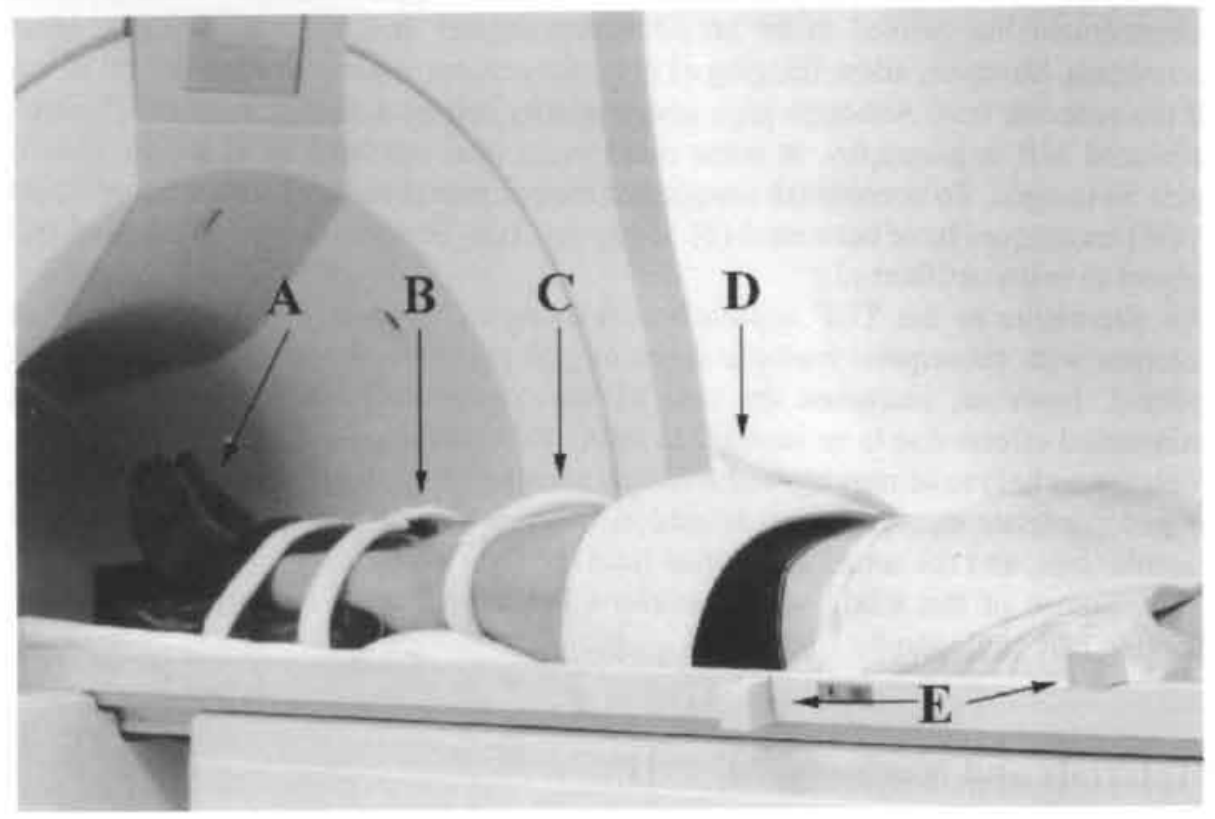

Figure 1. Photograph of a volunteer on the imaging table. The subject's feet are in the holder (A). Straps (B) are placed around the lower legs, two sandbags are used to stabilize the lower legs, and a strap (C) is placed around the upper legs, to prevent motion of the legs. A tight strap (D) placed around the thighs prevents wraparound artifacts. Premeasured stops on the stop stick (E) are used for exact repositioning of the table after table movement.

\section{Determination of Arrival-Time}

Dynamic gradient-recalled-echo MR imaging was performed with short repetition and echo times (21.7 and $3.4 \mathrm{msec}$, respectively). Forty-five axial images (matrix, $256 \times 102$; flip angle, $55^{\circ}$; field of view, $400-\mathrm{mm}$; section thickness, $15-\mathrm{mm}$ ) of the same location and with the same parameters were obtained consecutively through the abdominal aorta. The acquisition time for each section was 1.4 seconds.

By using dynamic imaging, the preparation phase for the acquisition is performed only once; thus, all sections were identical. A 100 -mm-thick caudal presaturation slab was used to suppress signal from inflowing venous blood, and a 80 -mm-thick cranial presaturation slab was used to prevent inflow effects from arterial blood. A test bolus of 1-mL of gadopentetate dimeglumine (Magnevist; Schering, Berlin, Germany) and a subsequent flush of $20-\mathrm{mL}$ of normal saline were administered by using an 18gauge intravenous cannula (Venflon; Ohmeda, Helsingborg, Sweden) in an antecubital vein to imitate the bolus of gadolinium used with the moving-bed infusion-tracking image. The contrast agent and subsequent normal saline flush were injected by using an MR-compatible injector (Spectris MR; MEDRAD, Pittsburgh, 
$\mathrm{Pa}$. The injection of the test bolus and the timing sequence were started simultaneously. The images were then evaluated for an increase in signal intensity in the abdominal aorta, which represents the arrival of the contrast agent. Arrival-fime of the bolus was calculated by multiplying the number of the section on which the elevated intra-aortic signal intensity was seen by the acquisition time per section.

\section{Determination of Vessel Anatomy}

To plan the volume used in moving-bed infusion-tracking imaging, sagittal maximum intensity projection (MIP) images were generated from images obtained with TOF sequences; these MIP images were used to obtain information about the arterial vessel course in the anterior-posterior direction (Fig. 2).

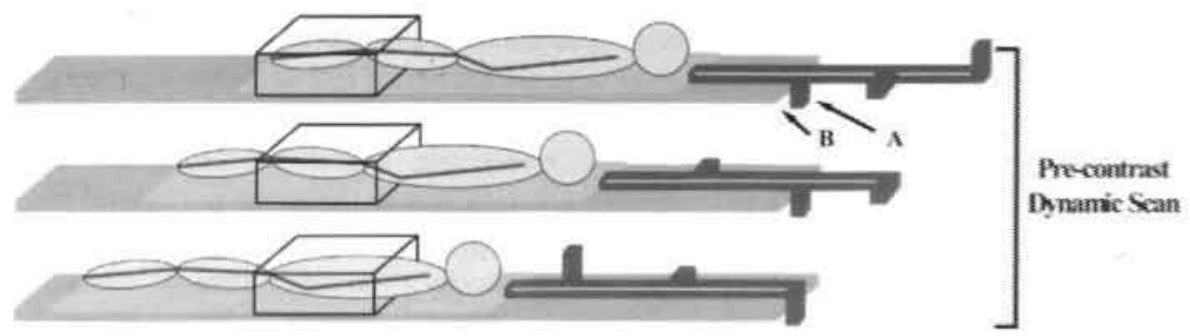

Start of injection of contrast material

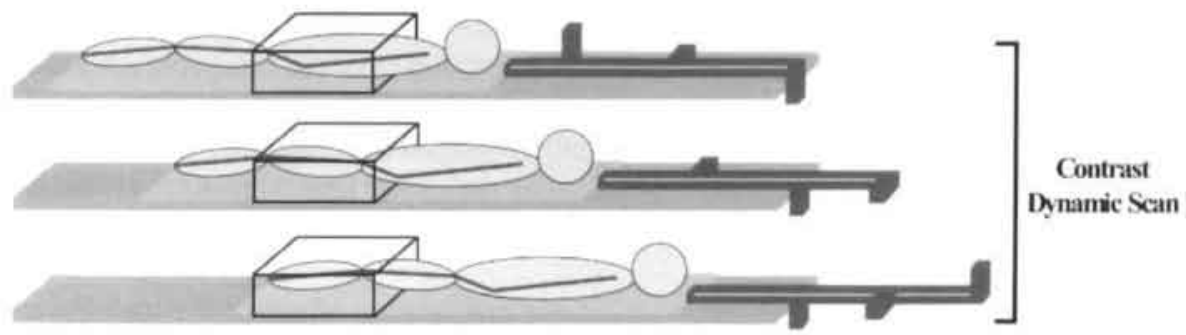

Figure 2. Schematic illustration shows sagittal views of a patient and the planned volumes for lower leg, upper leg and pelvic regions. The sequence of table movements is shown for unenhanced (Pre-contrast) imaging (top) and gadolinium-enhanced imaging (bottom). During table movement, the premeasured stop (A) of the stop-stick is pushed against the table support structure and the table is pushed against the end of the stop-stick (B). \{Movie-mode demonstration of the MoBI-track technique can be seen on the Image$\mathrm{CD}\}$.

The TOF sequences consisted of four noncardiac-synchronized sequences that covered the peripheral vascular tree from the aorta to the feet. For the TOF technique, a multiple two-dimensional magnetization-prepared gradient-recalled-echo (turbo "field-echo") sequence was used $\left(50^{\circ}\right.$ flip angle preceded by a $180^{\circ}$ inversion prepulse [3,4]; repetition time msec/echo time msec, 15.0/6.8; field of view, 250-mm; matrix size, 256x77; section thickness, 6-mm with a 2-mm intersection gap; one signal acquired). A 100 -mm-thick tracking venous presaturation slab with an 8-mmthick interslab gap caudal to the imaged section was used to suppress venous signal. The 160 sections ( 40 for each TOF sequence) were planned so the arterial vascular 
tree would be covered from the aorta to the feet. Acquisition of the TOF sequences and planning of the volumes for the moving-bed infusion-tracking images took approximately 20 minutes.

\section{Moving-Bed Infusion-tracking MR Angiography}

The moving-bed infusion-tracking MR angiographic sequence was a threedimensional gradient-recalled-echo (fast "field-echo") technique (14.1/6.1; flip angle, $50^{\circ} ; 32$ coronal sections acquired; section thickness, 3-mm [total volume thickness, 96-mm]). The images were acquired over-contiguously (interpolation of the 32 3mm-thick sections to 641.5 -mm-thick sections) in 42 seconds with a field of view of $500-\mathrm{mm}$ and a matrix size of $512 \times 171$, which resulted in a voxel volume of $8.4-\mathrm{mm}^{3}$. This sequence was implemented in a dynamic fashion to acquire three identical coronal volumes (ie, identical parameters and location in imager, but the patient was moved for each volume acquisition), each with 64 sections (192 sections for the entire dynamic imaging sequence). These coronal volumes were planned to cover the arteries of pelvic region. Elevation of the knees and ankles of the patient were optimized so the upper and lower leg arteries would run in the same horizontal plane as the pelvic arteries (Fig 2). This alignment of all peripheral arteries made it possible to image the entire peripheral vascular tree by moving the table into or out of the imager.

The dynamic study was acquired twice, once before infusion of contrast material and once during infusion. To acquire the unenhanced image, the table (with the patient) was pulled out of the imager; therefore, the first volume of the unenhanced study was of the lower legs. The table was then moved into the imager with a fixed table movement. To obtain accurate and reproducible table movements, a wooden stick with three premeasured stops, a so-called stop-stick (Figs 1,2), was used. A second unenhanced volume was then acquired of the upper legs. Again, the table was moved into the imager by using the stop-stick, and the next volume acquired covered the pelvic region.

After acquisition of the unenhanced study, the preparation phase of the gadoliniumenhanced study was started. The procedure was identical except the imaged regions were acquired in reverse order (pelvic, upper leg, and lower leg regions). After the preparation phase, $39-\mathrm{mL}$ of preheated gadopentetate dimeglumine was infused at a rate of $0.3-\mathrm{mL} / \mathrm{sec}$ by using the MR-compatible injector. The arrival-time of the contrast agent, as determined with the timing sequence plus 8 seconds (to ensure an even concentration of contrast material in the imaged volume), was used as an imaging delay between the start of contrast agent infusion and the start of the acquisition of the first volume.

After acquisition of the pelvic volume, the table was pulled out of the imager as fast as possible (approximately 3-4 seconds), and the stop-stick was used to position the table at exactly the same position used for acquisition of the second volume of the unenhanced image. The second volume of the gadolinium-enhanced study was then acquired. After the upper leg volume was acquired, the table was again pulled out of the imager as fast as possible, and the stop-stick was used to position the table at exactly the same position used to acquire the first volume of the unenhanced image 
(lower leg region). The third volume of the gadolinium-enhanced study was then acquired.

The two dynamic studies (a total of six coronal volumes) were reconstructed and sent to a workstation (SPARC station 5; Sun Microsystems, Mountain View, Calif) for postprocessing (Scil-image 1.2; University of Amsterdam, Faculty of Mathematics and Computer Science, The Netherlands), which consisted of sectionby-section subtraction of unenhanced volumes from corresponding gadoliniumenhanced volumes for all three anatomic regions. The subtracted data were then sent to another workstation (EasyVision; Philips Medical Systems). The left and right sides of the peripheral vascular tree were imaged separately by constructing six target MIP images that were rotated around the craniocaudal axis over a $150^{\circ}$ range $\left(0^{\circ}\right.$ [coronal MIP image], $30^{\circ}, 60^{\circ}, 90^{\circ}$ [sagittal MIP image], $120^{\circ}$, and $150^{\circ}$ ) for each side. The iliac arteries were also imaged by constructing six target MIP images rotated around the horizontal (left-right) axis $\left(0^{\circ}\right.$ [coronal MIP image], $30^{\circ}, 60^{\circ}, 90^{\circ}$ [axial MIP image], $120^{\circ}$, and $150^{\circ}$ ).

\section{Subjects}

Fifteen healthy volunteers, 8 men and seven women aged 19-41 years (mean, 28 years), with no known vascular pathologic conditions underwent MR angiography according to the procedure described earlier in this article.

After we had evaluated the feasibility of this method, 28 consecutive patients, 23 men and five women aged 31-82 years (mean, 62 years), underwent the same MR angiographic procedures as the volunteers. The patients had intermittent claudication, stenotic lesions of the iliac arteries (lumen reduction $250 \%$ ) demonstrated at Doppler ultrasonography (US), and/or were scheduled for conventional angiography. For the patients, conventional angiographic results served as the standard of reference. Approval was obtained from the medical ethical commission of the hospital, and written informed consent was obtained from all volunteers and patients.

\section{Conventional Angiography}

In all patients, conventional angiography was performed within 1 week of MR angiography by using an X-ray system (Diagnostic Arc; Philips Medical Systems) with a programmable stepping $\mathrm{C}$ arm, a film-changer (Puck; Elema-Schonander, Solina, Sweden), an add-on digital subtraction angiography system (Technicare DR 960-B; GE Medical Systems, Milwaukee, Wis), and a power injector for infusion of contrast material. All angiographic procedures were supervised by an experienced vascular radiologist (M.W.d.H.).

Conventional angiography was performed in 15 patients by puncturing the common femoral artery and placing a 5-F catheter in the distal aorta just above the bifurcation, if examination of two legs was necessary $(n=15)$, or by using a crossover approach, if examination of only one leg was necessary $(n=9)$. In 4 patients, conventional angiography was performed with anterograde infusion of contrast material in the common femoral artery. Nonionic contrast medium (iohexol, Omnipaque; Nycomed, 
Cork, Ireland) was used, with variable volumes and flow rates that depended on both the location of the tip of the catheter and the imaging system (conventional or digital subtraction angiography). Film hard copies were obtained in the posteroanterior direction; in most patients, additional posteroanterior and oblique views were obtained at digital subtraction angiography.

\section{Image Evaluation}

For evaluation purposes, the arterial tree was divided into the following segments: aorta, left and right common iliac arteries, external iliac artery, common femoral artery, superficial femoral artery, deep femoral artery, popliteal artery, anterior tibial artery, posterior tibial artery, and peroneal artery.

Because stenoses were all detected and graded on moving-bed infusion-tracking MIP images, image quality measurements were also performed on moving-bed MIP images. One observer (M.W.d.H.) evaluated the images in the volunteers for vessel visibility, which was classified as good, average, or poor. For the patients, the degree and length of a stenosis were assessed by two observers (P.J.E.H.M.K., J.M.A.v.E.) and were categorized with a five-point scale $(1=$ no abnormality or stenosis, with a lumen reduction of $0 \%$ to $20 \% ; 2=20 \%$ to $50 \%$ stenosis; $3=50 \%$ to $75 \%$ stenosis; 4 $=75 \%$ to $100 \%$ stenosis; $5=$ complete occlusion). Only the most severe stenosis per vessel segment was taken into account. Grading of stenoses was performed at a workstation with enlarged MIP images, by using an electronic calliper with $0.1-\mathrm{mm}$ accuracy. The two observers were unaware of the results of moving-bed infusiontracking MR angiography, conventional angiography, Doppler US, or the interpretation of the other observer. Because not all conventional angiograms in patients contained all regions of the peripheral vascular tree, only the vessel segments on moving-bed infusion-tracking MIP images with corresponding vessel segments on conventional angiograms could be compared.

The conventional angiograms were evaluated by two observers (M.W.d.H., J.M.A.v.E.) with regard to the degree and length of stenoses and were categorized by using the five-point scale. This was performed during separate sessions 4 weeks before the interpretations of moving-bed infusion-tracking MR angiograms. Evaluation of conventional angiograms was achieved with consensus about the results; a third observer (P.J.E.H.M.K.), who was a vascular surgeon, evaluated the images in cases where consensus was not reached.

Finally, moving-bed infusion-tracking MR angiographic and conventional angiographic results were compared directly by two radiologists (M.W.d.H., J.M.A.v.E.), who used a subjective quality score. For this purpose, both the movingbed MIP images and the conventional angiograms were evaluated simultaneously. Each vessel segment on the MIP images was interpreted in terms of contrast and the presence of artifacts. Contrast was defined as the visualized contrast between vessel and background and was categorized by using a scoring scale that ranged from -2 to 2 $(-2=$ much lower contrast compared with that on conventional angiograms, $-1=$ slightly lower contrast, $0=$ equal contrast, $1=$ slightly higher contrast, and $2=$ much higher contrast). Artifacts were defined as elements in the images that could disturb image interpretation (venous superposition, wraparound artifacts). 


\section{Statistical Analysis}

The linear weighted kappa statistic $(5,6)$ was used to assess interobserver agreement for stenosis grade. The $\kappa$ values can range from - 1 (no agreement) to 1 (perfect agreement). Interobserver agreement was classified as poor $(\kappa=0.00)$, slight ( $\kappa$ of $0.00-0.20$ ), fair ( $\kappa$ of $0.21-0.40$ ), moderate $(\kappa$ of $0.41-0.60$ ), substantial ( $\kappa$ of 0.61 0.80 ), or almost perfect ( $\kappa$ of $0.81-1.00)(7)$.

For findings in patients, sensitivity and specificity were calculated for presence of diseased vessel segments (lumen reduction $220 \%$ ) and for presence of hemodynamically significant stenoses (lumen reduction $250 \%$ ), with conventional angiographic findings as the standard of reference. The relationship between the results of moving-bed infusion-tracking MR angiography and conventional angiography with regard to the length and degree of a stenosis was assessed by using standard linear-regression analysis. To evaluate interobserver agreement for measurements of the length of stenoses, the Pearson correlation coefficient was calculated. This coefficient is a measure of the association between two random variables, given that these variables have a linear relationship (8).

\section{Results}

Adequate images were obtained in all volunteers and patients. Representative examples of the results of moving-bed infusion-tracking MR angiography in a volunteer and in a patient are shown in Figure 3. The observer who evaluated vessel visibility in volunteers concluded that all images provided good vessel visibility without disturbing artifacts. The location, number, and degree of stenotic lesions in patients as demonstrated on conventional angiograms are listed in Table 1.

Table 1. Stenoses Demonstrated at Conventional Angiography in 28 Patients and 15 Volunteers.

\begin{tabular}{llllll}
\multicolumn{5}{c}{ Stenosis Grade } \\
Artery & 1 & 2 & 3 & 4 & 5 \\
\hline Aorta & 14 & 2 & 0 & 0 & 0 \\
Common iliac & 21 & 4 & 3 & 3 & 3 \\
External iliac & 23 & 2 & 4 & 2 & 3 \\
Common femoral & 31 & 1 & 4 & 0 & 2 \\
Superficial femoral & 16 & 0 & 0 & 5 & 20 \\
Deep fernoral & 29 & 0 & 4 & 3 & 1 \\
Popliteal & 32 & 3 & 0 & 1 & 4 \\
Anterior tibial & 20 & 0 & 2 & 1 & 12 \\
Posterior tibial & 21 & 0 & 0 & 0 & 13 \\
Peroneal & 28 & 0 & 0 & 0 & 7
\end{tabular}

Before we compared moving-bed infusion-tracking MR angiographic results with conventional angiographic results, we excluded data of vessel segments that contained metallic stents. Interobserver agreement was almost perfect $(\kappa$ of $0.86 \pm$ 0.02 [1 standard deviation]) with regard to findings of degree of stenosis on moving- 
A

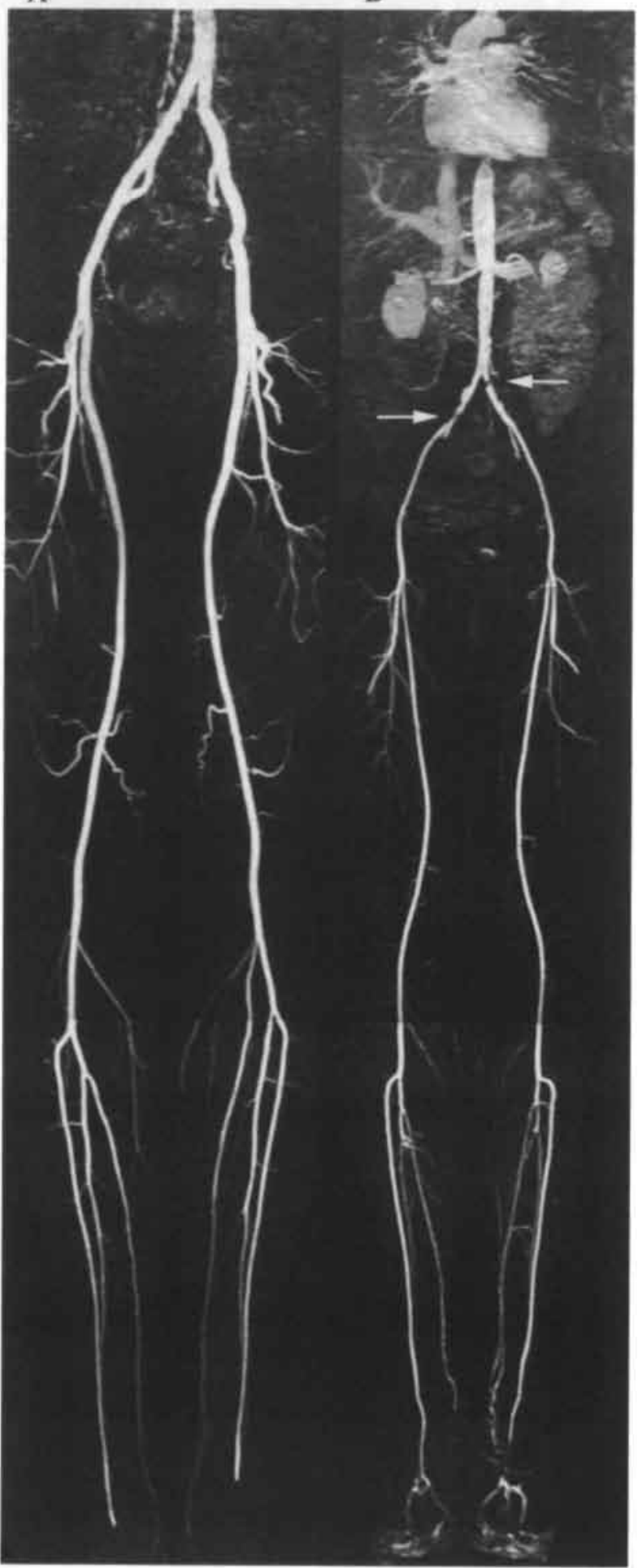

Figure 3. A: Coronal MR angiographic MIP images of peripheral vasculature in a 34-yearold, 202-cm-tall, healthy male volunteer and B: a 53-year-old, $158-\mathrm{cm}$-tall, female patient with atherosclerotic disease of the iliac arteries (arrows). These images show the robustness of the technique despite extreme patient variability. \{Three-dimensional views of the peripheral vasculature of these two subjects can be seen on the Image- $C D\}$. 
bed infusion-tracking MIP images. Interobserver agreement in terms of vessel segments is given in Table 2.

Table 2. Sensitivity, Specificity, and Interobserver Variability with Regard to Detection of Stenoses on MIP Images.

\begin{tabular}{|c|c|c|c|c|c|}
\hline \multirow[b]{2}{*}{ Artery } & \multicolumn{2}{|c|}{$\begin{array}{l}\text { Lumen Reduction } \\
220 \%\end{array}$} & \multicolumn{3}{|c|}{$\begin{array}{l}\text { Lumen Reduction } \\
250 \%\end{array}$} \\
\hline & $\begin{array}{l}\text { Sensitivity } \\
(\%)\end{array}$ & $\begin{array}{l}\text { Specificity } \\
(\%)\end{array}$ & $\begin{array}{l}\text { Sensitivity } \\
(\%)\end{array}$ & $\begin{array}{l}\text { Specificity } \\
(\%)\end{array}$ & $\begin{array}{l}\mathrm{K} \\
\text { value }\end{array}$ \\
\hline Aorta $(\mathrm{n}=16)$ & 75 & 76 & & 100 & 0.19 \\
\hline Common iliac $(\mathrm{n}=32)$ & 100 & 69 & 95 & 100 & 0.81 \\
\hline External iliac $(n=33)$ & 96 & 78 & 89 & 96 & 0.78 \\
\hline Common femoral $(n=38)$ & 86 & 79 & 67 & 94 & 0.85 \\
\hline Superficial femoral $(n=37)$ & 100 & 91 & 100 & 100 & 0.94 \\
\hline Deep femoral $(n=41)$ & 94 & 95 & 94 & 100 & 0.83 \\
\hline Popliteal $(n=40)$ & 88 & 91 & 100 & 97 & 0.84 \\
\hline Anterior tibial $(\mathrm{n}=35)$ & 100 & 80 & 97 & 100 & 0.88 \\
\hline Posterior tibial $(n=34)$ & 96 & 88 & 94 & 100 & 0.89 \\
\hline Peroneal $(n=35)$ & 79 & 84 & 79 & 100 & 0.78 \\
\hline
\end{tabular}

Sensitivity and specificity for detection and grading of diseased segments in all vessel segments (lumen reduction $220 \%$ ) were $91 \%$ and $92 \%$, respectively, for observer 1 and $99 \%$ and $75 \%$, respectively, for observer 2 . Sensitivity and specificity for detection and grading of hemodynamically significant stenoses in all vessel segments (lumen reduction $250 \%$ ) were $91 \%$ and $98 \%$, respectively, for observer 1 and $95 \%$ and $98 \%$, respectively, for observer 2 . These results are listed in Table 2 . Figure 4 shows the results of linear-regression analysis of the degree of a stenosis seen on moving-bed infusion-tracking MIP images and on conventional angiograms for all vessel segments.

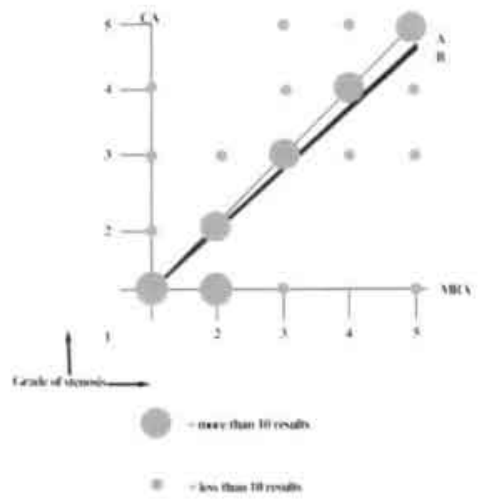

Figure 4. Graph shows results of the linear-regression analysis of findings of stenosis grade at moving-bed infusion-tracking MR angio-graphy (MRA) versus conventional angiography $(\mathrm{CA}) . \mathrm{A}=$ regression line based on findings by observer $\mathrm{I}, \mathrm{B}=$ regression line based on findings by observer 2 , dashed line $=$ perfect correlation between conventional and MR angiographic findings, large circle 10 or more results, small circle fewer than 10 results, $1=$ no abnormality $(0 \%$ to $20 \%$ stenosis), $2=20 \%$ to $50 \%$ stenosis, $3=50 \%$ to $75 \%$ stenosis, $4=75 \%$ to $100 \%$ stenosis, and $5=$ complete occlusion.

The length of hemodynamically significant stenoses and/or occlusions on conventional angiograms was $1-421-\mathrm{mm}$. Results of the two observers' assessments of length of such stenoses were highly correlated $(r=.99)$. Figure 5 shows the results of the linear-regression analysis of the length of stenoses seen on conventional angiograms and on moving-bed infusion-tracking MIP images. 


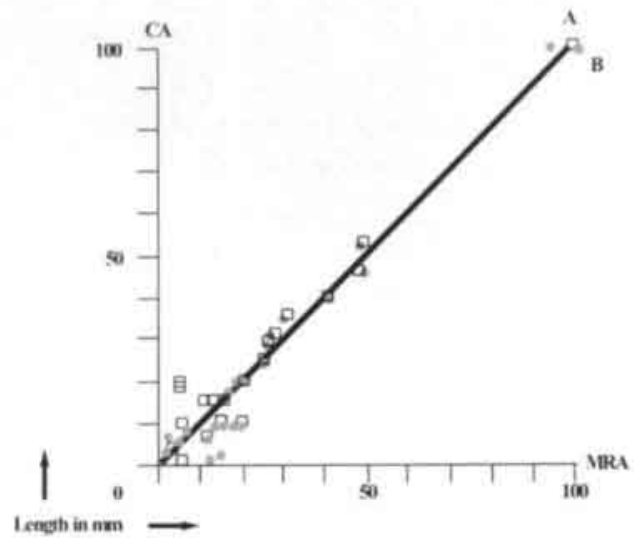

Figure 5. Graph shows results of the linear-regression analysis of findings of stenosis length at moving-bed infusion-trac-king MR angiography versus conventional angiography. The regression lines for observer $\mathrm{I}(\mathrm{A})$ and observer 2 (B) are almost identical to each other and to a perfect correlation between MR angiographic and conven-tional angiographic results. $O$ = finding by observer $1, \square=$ finding by observer 2 .

Table 3 lists the mean subjective contrast score per observer per vessel segment.

Table 3. Subjective Contrast Scores on Moving-Bed Infusion-tracking MR Angiograms.

\begin{tabular}{lll} 
Artery & Observer 1 & Observer 2 \\
\hline Aorta $(\mathrm{n}=16)$ & 0.19 & 0.00 \\
Common iliac $(\mathrm{n}=34)$ & 0.06 & 0.09 \\
External iliac $(\mathrm{n}=34)$ & 0.03 & 0.06 \\
Common femoral $(\mathrm{n}=38)$ & 0.00 & 0.03 \\
Superficial femoral $(\mathrm{n}=41)$ & 0.24 & -0.12 \\
Deep femoral $(\mathrm{n}=37)$ & 0.03 & -0.03 \\
Popliteal $(\mathrm{n}=40)$ & 0.38 & 0.05 \\
Anterior tibial $(\mathrm{n}=37)$ & 0.84 & 0.46 \\
Posterior tibial $(\mathrm{n}=37)$ & 0.76 & 0.49 \\
Peroneal $(\mathrm{n}=37)$ & 0.62 & 0.54
\end{tabular}

Note.-Subjective contrast (visual contrast between vessel and background) was scored on the basis of the following scale: -2 = much less contrast compared with that on conventional angiogram, $-1=$ slightly lower contrast than that on conventional angiogram, $0=$ contrast equal to that on conventional angiogram, $1=$ slightly higher contrast than that on conventional angiogram, 2 = much higher contrast than that on conventional angiogram.

\section{Discussion}

Several authors have demonstrated the usefulness of gadolinium-enhanced MR angiography for imaging of the aorta (9-14), iliac arteries (15-18), and lower leg arteries (16). To our knowledge, however, no study has yet been published in which the possibility has been demonstrated of imaging all three levels of the peripheral vascular tree in a short time (4 minutes) with only one bolus of contrast material and a conventional MR system.

Infusion of gadolinium-chelates over a longer period can cause two major problems at gadolinium-enhanced MR angiography: Venous enhancement can occur, and uptake of contrast material in surrounding tissue can result in lowered vessel-tobackground contrast. In another study (19), we compared gadolinium-enhanced MR 
angiograms of the pelvic region with conventional angiograms of the pelvic region after injection of $30-\mathrm{mL}$ of gadopentetate dimeglumine within 90 seconds; there were no troublesome artifacts of venous enhancement (20). By using three fast gadoliniumenhanced MR angiographic acquisitions (the three volumes of the dynamic study) right after each other, which extended the total acquisition time to 2 minutes 6 seconds, and by using a slow injection rate $(0.3-\mathrm{mL} / \mathrm{sec})$, the technique becomes a "gadolinium optimized tracking" technique, which was our former name for the moving-bed infusion-tracking technique (21).

The slow injection rate also has disadvantages. Because the elevation of arterial signal intensity depends on intra-arterial concentration of contrast material (20), the slower injection rate will also diminish vessel-to-background contrast. A subtraction technique with which background signal intensities can be lowered compensates for this effect. Moreover, the subtraction technique also compensates for the elevated background signal intensities (due to uptake of contrast material in tissue) in the upper and, especially, lower leg.

To demonstrate the effectiveness of subtraction in decreasing background signal intensities, Figure 6 shows a nonsubtracted and a subtracted MIP image of the lower legs.

A

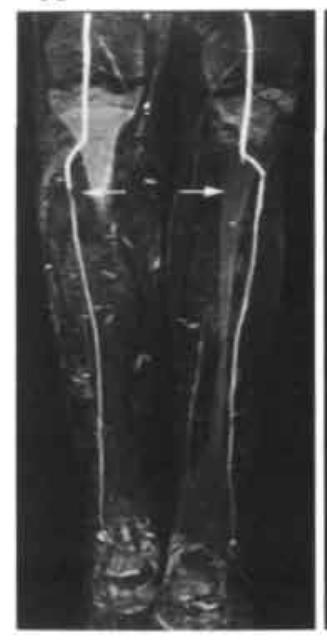

B

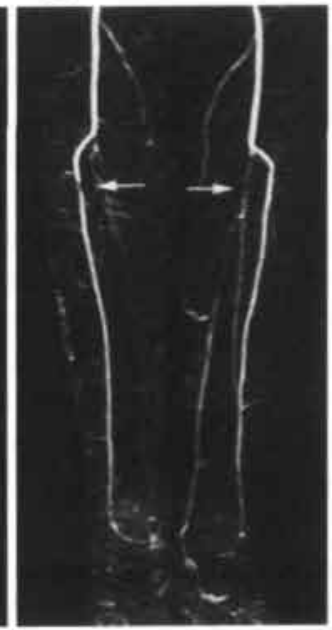

Figure 6. A: Nonsubtracted MIP image of the lower legs of a 54-year-old male patient with a right-sided single runoff vessel (anterior tibial artery) with a proximal stenosis (left arrow). On the left side, the anterior tibial artery is patent, but the posterior tibial artery (right arrow) seems to have a proximal occlusion, and the peroneal artery cannot be identified. B: Subtracted MIP image shows the same results on the right side as in A, but owing to the subtraction it can now be seen that the left leg has patent posterior tibial (arrow) and peroneal arteries, with severe stenoses in the tibioperoneal trunk and proximal posterior tibial artery.

An optimal subtraction of an unenhanced image from a gadolinium-enhanced image can be achieved only when the unenhanced image contains no or very little contrast material. With moving-bed infusion-tracking MR angiography, the unenhanced image was acquired before the administration of any gadopentetate dimeglumine (except the 1-mL used for the timing sequence).

Acquisition of all three unenhanced volumes before the gadolinium-enhanced study means that the table must be moved between acquisitions. This might cause artifacts in the subtraction due to misalignment. To obtain exact and reproducible alignment of these images, several precautions must be taken. First, one should ensure that the 
table be moved to fixed table positions. To achieve this, we used a so-called stopstick. This simple device, a wooden stick with premeasured stops affixed (Fig 1), is placed on the support structure on which the table lies. A stop is then pushed against the back of the support structure and the table is pushed against the end of the stopstick (Fig 2). To move from one region to another, the table is pushed into or pulled out of the imager by using the three stops on the stop-stick, as demonstrated in Figure 2. This stop-stick allows exact repositioning of the table, and thus of the patient, for both the unenhanced and the gadolinium-enhanced studies. Second, the patient's legs must be strapped together to prevent patient motion between acquisitions. For this purpose, we used sandbags on both sides of the legs and strapped together with the legs. This effectively prevented any leg motion between acquisitions despite fast table movement. Third, good patient instruction is essential. The patient should be made aware of the fast table movement between acquisitions of the six volumes so that he or she will not be startled when the table is suddenly moved.

To achieve high temporal resolution with a gadolinium-enhanced MR angiographic sequence on a conventional system, the repetition and echo times must be set to the shortest possible, and the rectangular field of view must be decreased to a minimum. By doing so, we were able to achieve an imaging time of 42 seconds per volume ( 2 minutes 6 seconds per dynamic study). However, this decrease in the field of view means that wraparound artifacts can occur, especially from the thighs. To prevent wraparound artifacts, we used a 200-mm-wide band strapped tightly around the thighs to prevent any artifacts that would disturb image interpretation.

A drawback to the use of a conventional imager is the limited volume thickness one can obtain with an image acquisition time of 42 seconds. This results in a relatively time-consuming procedure (20 minutes) for performance of TOF sequences and generation of their sagittal MIP images and to adjust the patient to the planned dynamic image volumes. The initial volume was planned to cover the arteries of the pelvic region. By elevating the knees and lower legs, upper and lower leg arteries were elevated. This meant that all peripheral arteries from the aorta to the feet were in a horizontal plane. By moving the bed and thus the arteries of another region (pelvic or lower or upper leg regions) into the imaged volume, we were able to image the entire vascular tree without repositioning of the acquired volume (in the anteriorposterior direction).

Because our imager could only acquire 256 sections per dynamic study, we had to acquire two dynamic studies (unenhanced and gadolinium enhanced). This can result in different amplifier settings, because a different preparation phase was performed for each dynamic study. Obviously, this might have resulted in suboptimal quality of the subtracted volumes. Subtraction of the different volumes could not be performed with the imager but had to be done with a separate workstation. This alternative route for data processing increased our postprocessing time to approximately 3 hours per patient. However, we are now using a more modern system (NT imager with EasyVision workstation; Philips Medical Systems), and postprocessing time has been decreased to approximately 15 minutes per patient.

On MIP images in the volunteers, no artifacts that disturbed interpretation were encountered. On MIP images in the patients, at least one observer interpreted the images of 33 of 351 vessel segments as having disturbing artifacts. 
The observers agreed about findings in only 12 of these segments. Three of these 12 artifacts were caused by metallic stents inserted during a previous vascular intervention. Two of these 12 artifacts were caused by misplanning of the volume for the gadolinium-enhanced images, which resulted in exclusion of part of the common femoral arteries from the imaged volume. Three of these 12 artifacts were wraparound artifacts. The wraparound artifacts occurred in the first two patients, whose thighs were not compressed by the thigh-strap. Also, one of these patients had two vessels in the lower leg that were not distinguishable from surrounding tissue due to a failed subtraction (lower leg rotation by a patient in whom no sandbags had been placed bilateral to the lower legs). The observers agreed only twice about disturbing artifacts due to venous superposition in a vessel segment, which means that there were unavoidable disturbing artifacts in only two of 351 vessel segments.

Because metallic stents are known to cause artifacts on gadolinium-enhanced MR angiograms (22), we excluded vessel segments that contained stents from our data before we compared the moving-bed infusion-tracking MIP images with conventional angiograms for grading of stenoses.

The moving-bed infusion-tracking MR angiographic technique yielded good results in all vessel segments. Table 2 demonstrates that the results were more or less independent of vessel size or location. We used two cut-off points: diseased vessel segments (lumen reduction $\geq 20 \%$ ) and hemodynamically significant stenoses (lumen reduction $250 \%$ ).

The comparison of moving-bed infusion-tracking MR angiograms and conventional angiograms in terms of diseased vessel segments can give a better impression of the spectrum of pathologic conditions encountered in peripheral arteries. However, this comparison resulted in lower specificity. This might be explained by the rectangular shape of the voxel used with the moving-bed infusion-tracking MR angiographic sequence, given the possible partial volume effects in the anteroposterior (sectionthickness) and left-to-right (low image-percentage) directions. This is also true for high grade stenoses (stenosis of $75 \%$ to $100 \%$ ), which sometimes manifested as small complete occlusions. Another explanation for the lower specificity might be the improved measurement accuracy on rotated and enlarged MIP images when the electronic caliper (with $0.1-\mathrm{mm}$ accuracy) was used, which revealed minor stenoses that were overlooked on conventional coronal or oblique angiographic views. With regard to the direct comparison between moving-bed infusion-tracking MIP images and conventional angiograms, the observers found the ability to rotate around a vessel segment at MR angiography useful. A typical example of this advantage can be seen in Figure 7, where a stenosis in the right common iliac artery was overlooked on conventional angiograms on both posteroanterior and oblique views. However, a sagittal MIP image of the gadolinium-enhanced image depicted this "undetectable" stenosis. The subjective contrast scores (Table 3 ) indicate that the mean scores of the two observers per vessel segment were equal to or better than those obtained with conventional angiography. This was particularly true for the lower legs, which showed consistently higher contrast scores for both observers. A typical example of the lower legs is seen in Figure 8. Most of the lower contrast scores found were due to artifacts (wraparound, misplanning of the volume, failed subtraction). Seven of 65 complete occlusions seen on conventional angiograms were "overlooked" on movingbed infusion-tracking MIP images. 
A

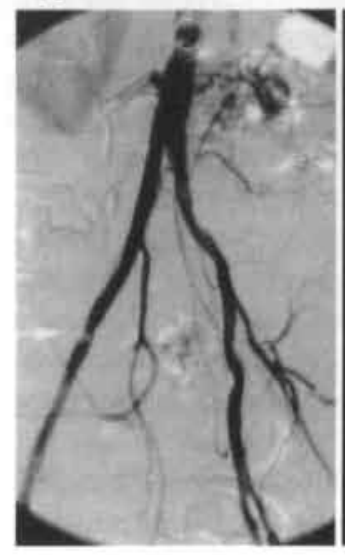

B

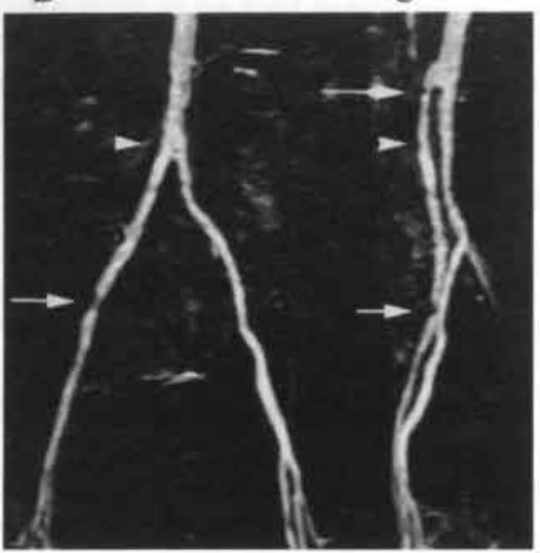

Figure 7. A: Oblique conventional angiographic view in a 46-year-old male patient depicts a stenosis (arrow) in the right external iliac artery but does not show the true anatomic position of the aortic bifurcation, owing to superposition of the right common iliac artery over the left common iliac artery. B: Coronal and C: sagittal moving-bed infusion-tracking $\mathrm{MR}$ angiographic MIP images, however, not only provide better views of the stenosis (straight arrows) in the right external iliac artery, but also demonstrate the false position of the bifurcation (arrowhead) and a stenosis (diamond-shaped arrow) of the right common iliac artery directly below the true anatomic position of the bifurcation. \{Three-dimensional views of the pelvic arteries can be seen on the Image-CD\}.

A

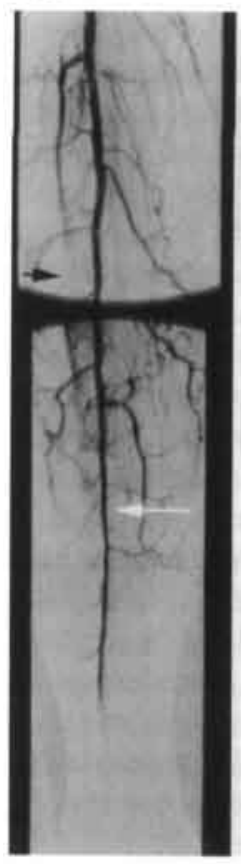

B

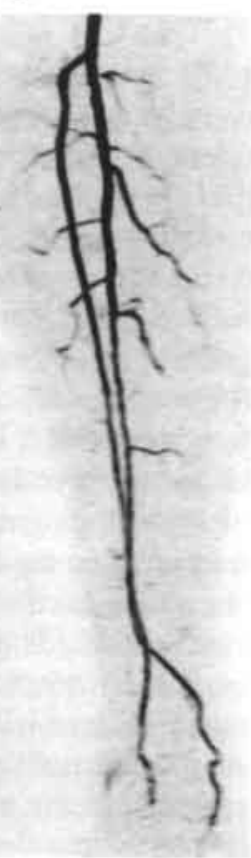

Figure 8. A: Conventional angiogram obtained with anterograde infusion of contrast material in a 54-year-old male patient. Two consecutive images depict the lower leg from the popliteal artery trifurcation to the ankle. A patent peroneal artery (white arrow), an anterior tibial artery with poor distal runoff (black arrow) and an occluded posterior tibial artery (arrowhead) are seen. B: The quality of the moving-bed infusion-tracking MR angiographic MIP image (inverse mode) is similar to that of the conventional angiogram in $\mathrm{A}$, and similar evaluation results were obtained. (Three-dimensional views of the lower leg arteries can be seen on the Image-CD .

These could be false-negative results. Figure 9a shows a conventional angiogram on which the left external iliac and common femoral arteries were not filled with contrast agent, probably because of mistiming. However, the corresponding moving-bed 
infusion-tracking MR angiographic MIP image (Fig 9b) depicts the apparently patent vessels.

A

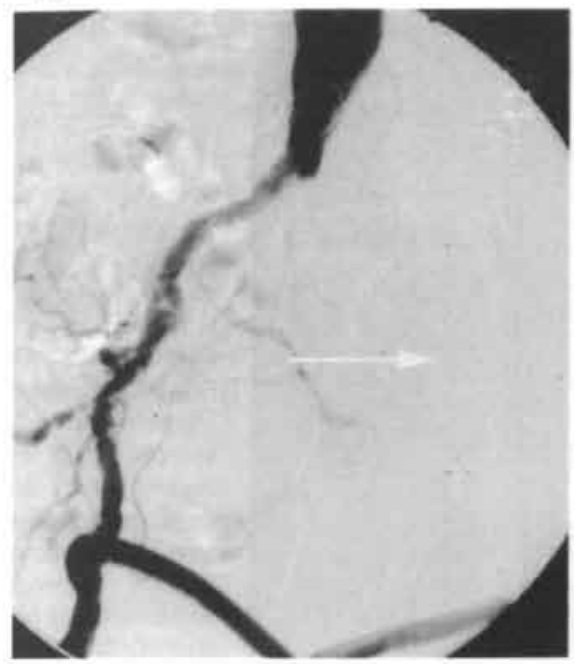

B

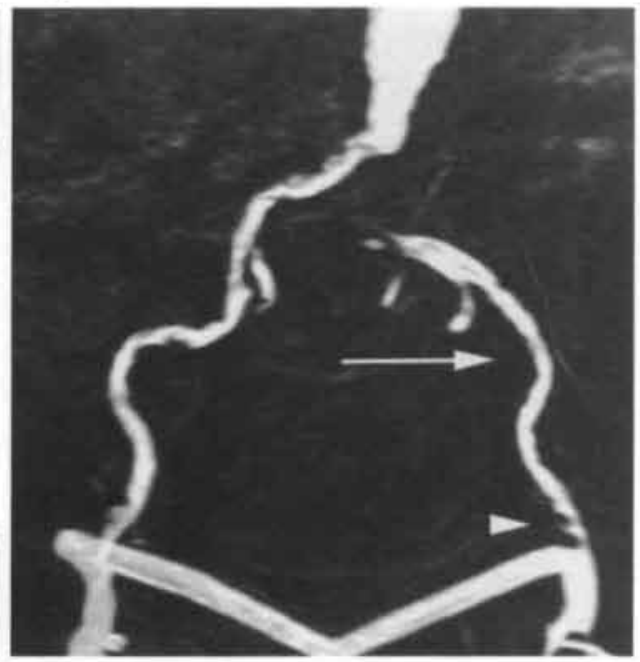

Figure 9. A: Conventional angiogram in a 79-year-old male patient shows complete occlusion of the left external iliac and common femoral arteries (arrow) due to mistiming. The longer imaging time (42 seconds) for moving-bed infusion-tracking MR angiography prevents problems due to mistimed postobstructive retrograde filling of arteries, as can be seen on the B: Moving-Bed Infusion-tracking MR angiographic MIP image, which shows patent left external iliac and common femoral arteries (arrow), with two severe stenoses (arrowhead) just proximal to the junction of the bypass and the left common femoral artery. (Three-dimensional views of the pelvic arteries can be seen on the Image-CD).

Similar phenomena were seen for the other "overlooked complete obstructions", thought to indicate occlusion on conventional angiograms but not on moving-bed infusion-tracking MIP images. More study is needed, with surgical exploration as the standard of reference, to evaluate whether these findings were overlooked occlusions or false-negatives.

In another study (19), we concluded that complete occlusions seen at gadoliniumenhanced MR angiography might best be evaluated by using both nonsubtracted and subtracted images. This was due to the fact that the subtracted images can lack sufficient anatomic information, which sometimes results in misinterpretation of vessel anatomy. Figure 10 demonstrates this effect in the upper femoral region. On the pelvic image alone, one might think that the superficial femoral arteries are unaffected. However, by looking at the comprehensive picture of the pelvic and upper leg region, one can immediately see that both superficial femoral arteries are completely occluded. With regard to length measurements, the results of linearregression analysis (Fig 5) show that findings from moving-bed infusion-tracking MR angiography are in excellent accordance with those of conventional angiography. Again, this might be explained by the voxel dimensions, for which the best resolution is achieved in the craniocaudal direction.

All symptomatic patients with arteriosclerotic peripheral vessel disease need an extensive diagnostic work-up, including noninvasive tests, for an accurate diagnosis 
to be established. Precise definition of vessel anatomy is mandatory for treatment planning and for the decision about the most appropriate type of intervention.

A

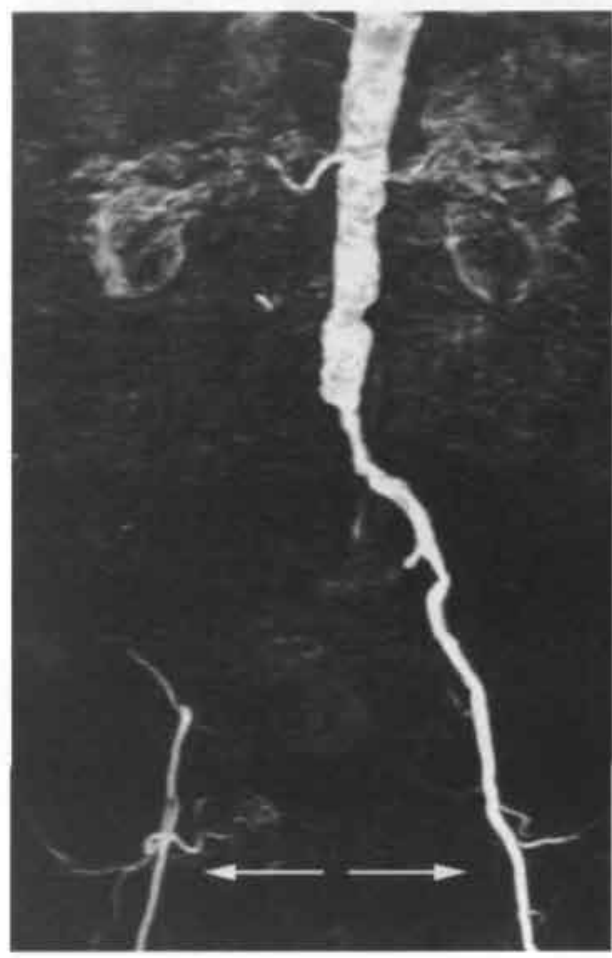

B

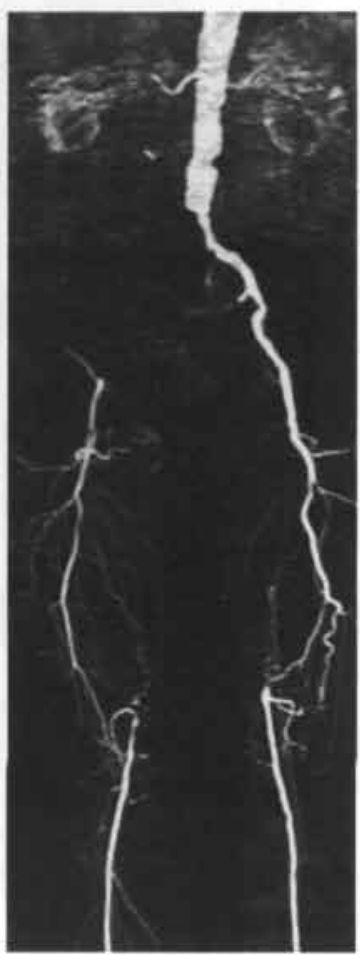

Figure 10. Moving-bed $M R$ angiographic MIP images in a 73year-old male patient. A: Image of the pelvic region appears to depict normal superficial femoral arteries (arrows), but the B: comprehensive image of the pelvic and upper leg regions clearly demonstrates that both superficial femoral arteries are completely occluded. \{Threedimensional views of the peripheral vasculature can be seen on the Image-CD .

Moving-bed infusion-tracking MR angiography is a promising technique for fulfillment of these needs. However, more patients must be evaluated with this technique to establish its value in imaging of the peripheral vascular tree.

Further improvement of moving-bed infusion-tracking MR angiography is to be expected with the use of newer (eg, faster) imagers. Use of identical amplifier settings between unenhanced and gadolinium-enhanced studies can improve subtracted image quality. For this purpose, it should be possible to obtain more than 256 sections per dynamic study, which is a limitation of our imager that forced us to acquire two dynamic studies (unenhanced dynamic and gadolinium-enhanced dynamic studies). It should also be possible to use three amplifier settings per region (lower leg, upper leg, and pelvic region). Better hardware, such as specific extremity coils (instead of the body coil), will improve the signal-to-noise ratio remarkably. Faster imaging times can lead to higher spatial resolution or thicker volume acquisition.

Because sensitivity and specificity for detecting and grading hemodynamically significant stenoses are already approximately $95 \%$ (excluding false-negative results), we think that a thicker volume acquisition would be the primary goal for improving 
moving-bed infusion-tracking MR angiography. Acquisition of a thicker volume may eliminate the need for exact planning of volumes. This would reduce overall patienthandling time considerably, because the time needed for acquisition of TOF images could be eliminated. Because accurate timing of the bolus is important (23), the timing sequence cannot be eliminated unless an automatic bolus detection system such as the one recently described by Foo et al (24) is used. Imaging time can be reduced to include the time of moving-bed infusion-tracking MR angiography alone (4 minutes) with only one bolus of contrast material.

In conclusion, moving-bed infusion-tracking MR angiography is a fast, simple, and robust technique with which the entire peripheral vascular tree can be imaged with a conventional 1.5-T MR imager. Our results in patients are promising. In the iliac and femoral arteries, and particularly in the lower leg arteries, results obtained with this technique can even compete with those of conventional angiography. With faster imagers, overall patient-handling time may be reduced to 4 minutes.

\section{Acknowledgments}

We gratefully thank Philips Medical Systems (Best, The Netherlands) and Marc Kouwenhoven, MSc, in particular, for their efforts to implement this technique with our imager. 


\section{References}

1. Prince MR. Body MR angiography with gadolinium contrast agents. Magn Reson Imaging Clin N Am 1996; 4:11-24.

2. Yucel EK, Kaufman JA, Geller SC, Waltman AC. Atherosclerotic occlusive disease of the lower extremity: prospective evaluation with two-dimensional time-of-flight MR angiography. Radiology 1993; 187: 637-641.

3. Ho KY, de Haan MW, Oei TK, et al. MR angiography of the iliac and upper femoral arteries using four different inflow techniques. AJR 1997; 169:45-53.

4. Yucel EK, Silver MS, Carter AR MR angiography of normal pelvic arteries: comparison of signal intensity and contrast-to-noise ratio for three different inflow techniques. AJR 1994; 163:197-201.

5. Cohen J. A coefficient of agreement for nominal scales. Educ Psychol Meas 1960; 20:37-46.

6. Cohen J. Weighted kappa: nominal scale agreement with provision for scaled disagreement or partial credit. Psychol Bull 1968; 70:213-230.

7. Landis JR, Koch GG. The measurement of observer agreement for categorical data. Biometrics 1977; 33:159-174.

8. Kleinbaum DG, Kupper LL, Muller KE. Applied regression analysis and other multivariable methods. 2nd ed. Boston, Mass: PWS-Kent, 1988; 80.

9. Leung DA, McKinnon GC, Davis CP, Pfammatter T, Krestin C;P, Debatin JF. Breathhold, contrast-enhanced, three-dimensional MR angiography. Radiology 1996; 200:569-571.

10. Holland GA, Dougherty L, Carpenter JP, et al. Breath-hold ultrafast threedimensional gadolinium-enhanced MR angiography of the aorta and the renal and other visceral abdominal arteries. AJR 1996; 166: 971-981.

11. Prince MR, Narasimham DL, Stanley JC, et al. Breath-hold gadolinium-enhanced MR angiography of the abdominal aorta and its major branches. Radiology 1995; 197:785-792.

12. Laissy JP, Soyer P, Tebboune D, Tiah D, Hvass U, Menu Y. Abdominal aortic aneu rysms: assessment with gadolinium-enhanced time-of-flight coronal MR angiography (MRA). Eurj Radiol 1995; 20:1-8.

13. Prince MR. Gadolinium-enhanced MR aortography. Radiology 1994; 191:155-164.

14. Prince MR, Yucel EK, Kaufman JA, Harrison DC, Geller SC. Dynamic gadoliniumenhanced three-dimensional abdominal MR arteriography. JMRI 1993; 3:87 7-88.

15. Snidow JJ, johnson MS, Harris VJ, et al. Three-dimensional gadolinium-enhanced MR angiography for aortoiliac inflow assessment plus renal artery screening in a single breath hold. Radiology 1996; 198: 725-732.

16. Douek PC, Revel D, Chazel S, Falise B, Villard J, Amiel M. Fast MR angiography of the aortoiliac arteries and arteries of the lower extremity: value of bolus-enhanced, whole-volume subtraction technique. AJR 1995; 165:431-437.

17. Snidow JJ, Aisen AM, Harris VJ, et al. Iliac artery MR angiography: comparison of three-dimensional gadolinium-enhanced and two-dimensional time-of-flight techniques. Radiology 1995; 196:371-378.

18. Sivananthan UM, Ridgway JP, Bann K, et al. Fast magnetic resonance angiography using turbo-FLASH sequences in advanced aortoiliac disease. Br j Radiol 1993; 66: 1103-1110.

19. Ho KYJAM, de Haan MW, Kessels AGH, Kitslaar PJEHM, van Engelshoven JMA. Peripheral vascular tree stenoses: detection with subtracted and nonsubtracted MR angiography. Radiology 1998;206: 673-681. 
20. Strouse $\mathrm{Pj}$, Prince MR, Chenevert TL. Effect of the rate of gadopentetate dimeglumine administration on abdominal vascular and soft-tissue MR imaging enhancement patterns. Radiology 1996; 201:809-816.

21. Ho KY, Leiner T, de Haan MW, van Engelshoven JMA. Gadolinium optimized tracking technique: a new MRA technique for imaging the peripheral vascular tree from aorta to the foot using one bolus of gadolinium (abstr). In: Proceedings of the Fifth Meeting of the International Society for Magnetic Resonance in Medicine. Berkeley, Calif: International Society for Magnetic Resonance in Medicine, 1997: 203.

22. Laissy JP, Grand C, Matos C, Struyven J, Berger JF, Schouman-Claeys E. Magnetic resonance angiography of intravascular endoprostheses: investigation of three devices. Cardiovasc Intervent Radiol 1995; IS:360-366.

22. Prince MR, Chenevert TL, Foo TK, Londy Fj, Ward JS, Maki JH. Contrast-enhanced abdominal MR angiography: optimization of imaging delay tiine by automating the detection of contrast material arrival in the aorta. Radiology 1997; 203:109-114.

24. Foo TK, Saranathan M, Prince MR, Chenevert TL. Automated detection of bolus arrival and initiation of data acquisition in fast, three-dimensional, gadoliniumenhanced MR angiography. Radiology 1997; 203:275-280. 


\title{
Chapter 6
}

\section{Analysis of Image Interpretability in Contrast- enhanced MR Angiography using the MoBI-track technique}

\author{
Submitted
}

Authors: Ho K.Y.J.A.M., Leiner T., Kessels A.G.H., de Haan M.W., Kouwenhoven M., van Engelshoven J.M.A. 



\section{Abstract}

Introduction: The Moving-Bed Infusion-tracking technique (MoBI-track) is a contrast-enhanced peripheral MR angiographic technique capable of imaging the entire peripheral vascular tree from aorta down to the feet in several minutes. However, in a selected number of cases, image interpretability can be suboptimal due to various causes.

Purpose: To retrospectively evaluate image interpretability of peripheral contrastenhanced MR Angiography using the MoBI-track technique and to define technique and patient related factors which may influence image interpretability.

Subjects and materials: One-hundred-and-thirty-one patients divided into two consecutively examined groups were retrospectively evaluated for the presence of factors disturbing image interpretability. Using a three-point subjective interpretability score (good, suboptimal and poor interpretability), all vessel segments (29 per patient) were evaluated. Using a multivariate logistic regression analysis, the influence of different technique and patient related factors was examined (inclusion period, inflow pathology, Fontaine classification, height of the lower legs, age, lower leg pathology, sex, and venous enhancement in the lower legs).

Results: Poor image interpretability was found in $2.5 \%$ of all evaluated vessel segments. Poor image interpretability occurred mainly in the lower legs $(5 \%$ of all lower leg vessel segments). In the pelvic and upper leg vessel segments hardly any poor vessel interpretability was found $(0 \%$ and $1 \%$, respectively). Main disturbing factor for image interpretation in the lower legs was venous enhancement. No other statistically significant factors were identified.

Conclusion: MoBI-track MR angiography is capable of producing good image interpretability in a majority of patients and vessel segments. If venous enhancement in the lower legs can be prevented by using more dedicated hard- and software equipment, image interpretability can become near perfect. 


\section{Introduction}

In recent years, contrast-enhanced peripheral MR angiographic techniques have been developed to visualize the whole peripheral arterial tree form renal arteries down to the feet [1-4]. Since 3 years we have been using the Moving-Bed Infusion-tracking technique (MoBI-track) which allowed us to image the entire peripheral vasculature within 4 minutes injecting only one single bolus of gadolinium $(30-\mathrm{mL}$ of Gd-DTPA $0.5-\mathrm{mmol} / \mathrm{mL}$ ). The slowly infused (very long ) bolus is tracked down the legs, taking advantage of the relatively long time between arterial and venous enhancement in the lower legs (arterial-venous window varies per patient up to $120-150 \mathrm{sec}$ ). With this method a 93\% sensitivity and $98 \%$ specificity for detecting and grading hemodynamically significant stenoses could be demonstrated [4]. However, in a selected number of cases, image interpretability is suboptimal due to various causes.

The purpose of this study was to evaluate image interpretability of peripheral contrast-enhanced MR Angiography using the MoBI-track technique and to define technique and patient related factors which may influence image interpretability and to give suggestions for improvements.

\section{Subjects and Methods}

One hundred-and-thirty-one patients ( 84 men and 47 women; mean age 63 years, range 17-89), most of them with clinical symptoms of peripheral vascular obstructive disease, were included. The Fontaine classification was used to categorize the severity of clinical symptoms [5]. This classification is a four-point scale for clinical severity of patients complaints; Fontaine score I: no subjective complaints. Fontaine score II: complaints of intermittent claudication. Fontaine score III: patients with rest pain in the legs. Fontaine score IV: patients with rest pain and ulceration of the legs. Seven of these 131 patients had Fontaine classification I but there was clinical suspicion of peripheral vascular obstructive disease, 101 patients had Fontaine classification II, 15 patients had Fontaine classification III and 8 patients had Fontaine classification IV. Of all patients included, one had an amputation of his left lower leg. Twenty-four patients underwent MR angiography as part of their diagnostic workup, because of contra-indications for diagnostic X-ray angiography (20 patients) or because diagnostic X-ray angiography could not demonstrate any distal runoff vessels (4 patients). The remaining patients (107) underwent peripheral MR angiography because they were included in one of the clinical trials concerning peripheral MR angiography running at our department. The vascular abnormalities as diagnosed in the original radiological reports were classified using a scoring scale called the vascular pathology scale (VPS), discriminating between lumen narrowing from 0 to $50 \%$ (score 0 ), and from 50 to $100 \%$, including complete occlusions (score 1).

MR examinations were done during two separate periods. From October 1997 to March 1998 eighty-eight consecutive patients were examined and from July to November 1998 forty-three consecutive patients. The period in between, no patients were included because new technical improvements were tested. The characteristics 
of both groups of patients concerning age, sex, Fontaine classification and VPS scores in pelvic, upper and lower leg regions were examined to evaluate any differences between the two groups.

Technical improvements introduced in between the inclusion periods consisted of the use of a dedicated lower extremity coil (Rigid T-shaped synergy coil containing 3 quadrature phased array elements including a nonuniformity correction filter to compensate for signal loss further away from the coil [6]), and an upgrade of our scanner from a 1.5 T MR system (Powertrak 3000, release 5; Philips Medical Systems, Best, the Netherlands) with a maximum gradient strength of $15 \mathrm{mT} / \mathrm{m}$ and a rise time of $0.5 \mathrm{msec}$ to a $1.5 \mathrm{~T}$ MR system (Powertrak 6000, release 6.1; Philips Medical Systems, Best, the Netherlands), with a maximum gradient strength of 21 $\mathrm{mT} / \mathrm{m}$ and a rise time of $0.3 \mathrm{~ms}$.

Approval for the different studies was obtained from the medical ethics commission of our hospital, and informed consent was obtained prior to the examination from those patients who were not examined for clinical purposes.

\section{MR Angiography}

An optimized form of the recently described MoBI-track technique was used. Optimization of MoBI-track MR angiography, implemented by the scanner manufacturer (Philips Medical Systems), consisted of semi-automatic table movement without the need for a stopping and repositioning device, angulation of the different volumes per station as well as acquiring different amplifier settings per station.

The acquisition protocol started with three fast low resolution time-of-flight (TOF) sequences of the pelvic, upper leg and lower leg stations to obtain sagittal maximum intensity projection (MIP) images for information about the arterial vessel course in the anteroposterior direction in order to plan the volumes of interest for the contrastenhanced sequence. The contrast-enhanced sequences were slightly different between the two periods of patient inclusion due to optimization of parameters per scanner. Each sequence was chosen to achieve the highest resolution and signal-to-noise ratio and contrast-to-noise ratio (SNR and CNR) possible for a given temporal resolution $(35-40 \mathrm{sec})$. The basic sequence was a three-dimensional gradient-recalled echo (fast "field-echo") technique using a TR of 7-10-msec and a TE of 2-3-msec with a flip angle of $25-35^{\circ}$. Thirty-two to 55 coronal partitions with a partition thickness of 2.53-mm (total volume thickness $100-165-\mathrm{mm}$ ) were acquired in $35-40$ seconds with a FOV of 450-mm and a matrix size of $512 \times 150-175$ resulting in voxel volumes of 6$10-\mathrm{mm} 3$. The sequence used was implemented in a dynamic scan with two dynamic subscans which each acquired three stacks. These coronal stacks were planned to cover the arteries of pelvic, upper leg and lower leg arteries (fig. 1). The first dynamic subscan was acquired before infusion of contrast material (mask scan), and the second during infusion of $30-\mathrm{mL}$ of contrast material (Magnevist ${ }^{*}$, Schering, Berlin, Germany in 93 patients and Omniscan ${ }^{\star}$, Nycomed Amersham, Oslo, Norway in 38 patients) using a remote controlled MR compatible power injector (Spectris MR; MEDRAD, Pittsburgh, PA). 

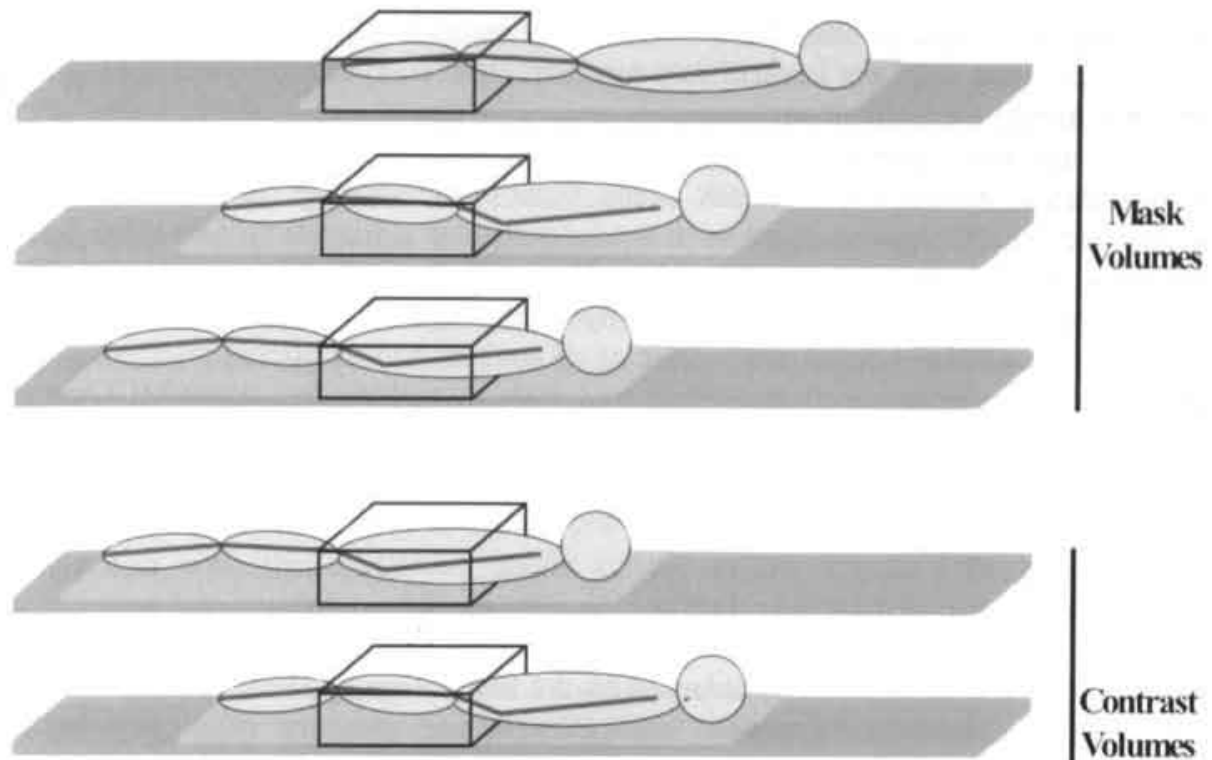

Contrast

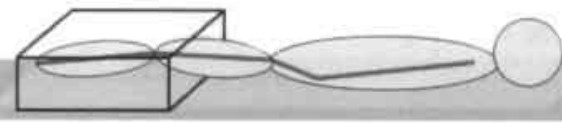

Volumes

Figure 1. Schematic illustration of the principal of the Moving-Bed Infusion-tracking (MoBI-track) technique. The sequence of table movements is shown for unenhanced (mask volumes) imaging (top) and gadolinium-enhanced imaging (bottom). The table is moved automatically by the scanner software.

Contrast material and subsequent flush of $25-\mathrm{mL}$ of normal saline $(\mathrm{NaCl} 0.9 \%)$ were administered with an injection rate of $0.3-0.4-\mathrm{mL} / \mathrm{sec}$ via an 18 gauge intravenous cannula (18G, venflon 2, Ohmeda, Helsingborg, Sweden) in an antecubital vein. For all patients a fixed timing delay of 55 seconds between start of injection of contrastmaterial and start of data acquisition was used. Both mask and contrast stacks of the pelvic station were acquired during breathhold. Patients legs were immobilized using a dedicated footboard or the lower extremity coil with integrated foot immobilizer (fig. 2).

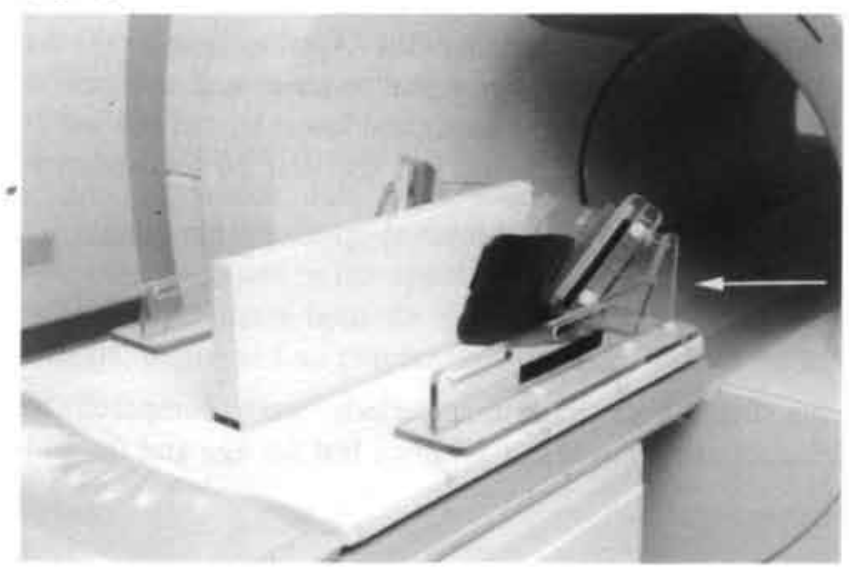

Figure 2. Image of the rigid T-shaped dedicated lower extremity coil that contains 3 quadrature phased array elements. Arrow points at the integrated foot immobilizer. 
Total patient handling time for the entire examination was approximately $15-20$ minutes. As data were not immediately reconstructed (delayed reconstruction), 3 to 4 patients could be examined per hour.

After the two dynamic subscans, with in total 6 coronal volumes were reconstructed, subtraction of mask scans from corresponding contrast scans was performed. The subtracted data was sent to a computer workstation (Sun Ultra-Sparc 30, Sun Microsystems Inc., Mountain View, CA) to be post-processed with EasyVision software (Philips Medical Systems, Best, The Netherlands). Whole volume coronal MIP images were made of all stations and additionally left and right peripheral vascular tree were processed separately by making five target MIP images spinned around the cranio-caudal axis over $150^{\circ}\left(0^{\circ}\right.$ [coronal MIP], $30^{\circ}, 60^{\circ}, 90^{\circ}$ [sagittal MIP], $120^{\circ}$ and $150^{\circ}$ ).

\section{Image Evaluation and Interpretation}

All images were retrospectively assessed by the first author concerning overall image interpretability, amount of venous enhancement and presence of misregistration artifacts. For this purpose, the arterial tree on the MIP images was divided into the following segments: aorta, both right and left sided common iliac and external iliac arteries, common, deep and superficial femoral arteries (the latter was divided in an upper, middle and lower part), popliteal arteries, anterior, posterior tibial and peroneal arteries (the latter three divided into an upper and lower part). All vessel segments were assessed and categorized using two scoring scales. The first scale called the subjective interpretability scale (SIS), was used to score subjective vessel segment interpretability. Score $0=$ good vessel segment interpretability, score $1=$ suboptimal vessel segment interpretability, score 2 = poor vessel segment interpretability. The second scale called the venous enhancement scale (VES), was used to score the amount of venous enhancement next to the evaluated vessel segment. Score $0=$ no venous enhancement, score 1 = some venous enhancement, score 2 = much venous enhancement.

In order to evaluate the effect of movement in between the acquisition of mask and contrast-enhanced volumes, all coronal MIP images were evaluated for the presence of misregistration artifacts after subtraction.

Furthermore, to evaluate the technical improvements (dedicated lower extremity coil and scanner update) introduced during the three months (April to June 1998) that no patients were included, we compared the mean signal-to-noise and contrast-tonoise ratios for nondiseased vessel segments in the upper and lower legs of the last 20 (consecutive) patients from the first inclusion period and the first 20 (consecutive) patients from the second inclusion period.

\section{Statistical Analysis}

The two groups of patients from both inclusion periods were compared for statistically significant differences using the Mann Whitney test for age and the Chi- 
square test for sex, Fontaine classification and VPS scores in pelvic, upper and lower leg regions.

The effect of various patient or procedure dependent parameters on vessel segment interpretability were studied using a multi-variate logistic regression model. As dependent variable we used the dichotomized SIS results (good and suboptimal versus poor vessel segment interpretability) of the lower legs. The independent variables were: inclusion period; two groups of patients based on the inclusion period (Oct 1997-Mar 1998 versus Jul-Nov 1998), inflow pathology; dichotomized for the maximum VPS per leg ( 0 versus 1$)$ in either one of the following inflow vessels per leg: aorta, common iliac, external iliac, common femoral, deep femoral, superficial femoral and popliteal arteries, Fontaine classification; dichotomized for Fontaine 1 and 2 versus Fontaine 3 and 4, height of the lower legs; dichotomized into two equal groups of patients according to the height of the middle of the peroneal artery in comparison with the maximum height of the inflow vessels (see fig. 3), age; categorized into tertiles ( $\leq 58 \mathrm{yrs}, 59-65 \mathrm{yrs}$ and $\geq 66 \mathrm{yrs}$ ), lower leg pathology; dichotomized for VPS 0 versus VPS 1 in either one of the following lower leg arteries per leg: anterior tibial, posterior tibial and peroneal artery, sex, and enhancement of the lower leg veins; dichotomized for VES 0 and 1 versus VES 2 per lower leg artery.

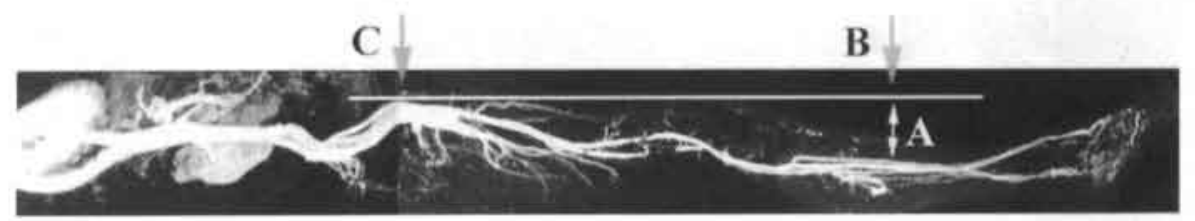

Figure 3. Image demonstrates how the height $(\mathrm{A})$ of the middle of the peroneal artery $(\mathrm{B})$ as compared to the maximum height of the inflow (pelvic and upper leg) arteries (C) was measured. Hypothesis was; the lower the lower leg arteries as compared to the inflow arteries, the more venous enhancement would occur in the lower legs.

SNR and CNR, calculated for the upper leg and lower leg station to evaluate the different technical improvements introduced between the two periods of inclusion, were compared using a linear regression model with the inclusion period and imaged station as independent variables, and patients whose data was used for these calculations were separately compared for above mentioned patients characteristics.

\section{Results}

The two groups of patients (from both inclusion periods) as well as the two subgroups of patients whose data were used to calculate SNR and CNR, did not differ statistically significantly. In total 3793 vessel segments were evaluated, 1179 in the pelvic station, 1048 in the upper leg station and 1566 in the lower leg station.

Table 1 tabulates both the results of the SIS and the results of the VES for all patients, expressed as percentages of total number of assessed vessel segments per station. 
Table 1. Subjective interpretability and venous enhancement scores tabulated as percentages of the total number of evaluated vessel segments per station for all 131 patients.

\section{Subjective interpretability scores} (SIS)
Venous enhancement scores (VES)

\begin{tabular}{lllllll} 
& 0 & 1 & 2 & 0 & 1 & 2 \\
\hline Pelvic station & $97 \%$ & $3 \%$ & $0 \%$ & $91 \%$ & $8 \%$ & $1 \%$ \\
Upper leg station & $98 \%$ & $1 \%$ & $1 \%$ & $75 \%$ & $17 \%$ & $8 \%$ \\
Lower leg station & $91 \%$ & $4 \%$ & $5 \%$ & $90 \%$ & $2 \%$ & $8 \%$
\end{tabular}

SIS 0: good vessel segment interpretability, SIS 1: suboptimal vessel segment interpretability, SIS 2: poor vessel segment interpretability. VES 0: no venous enhancement next to the vessel segment, VES 1: some venous enhancement next to the vessel segment, VES 2: much venous enhancement next to the vessel segment.

Representative examples of coronal MIP images with good, suboptimal and poor vessel segment interpretability as well as with none and much venous enhancement are shown in figure 4.

A

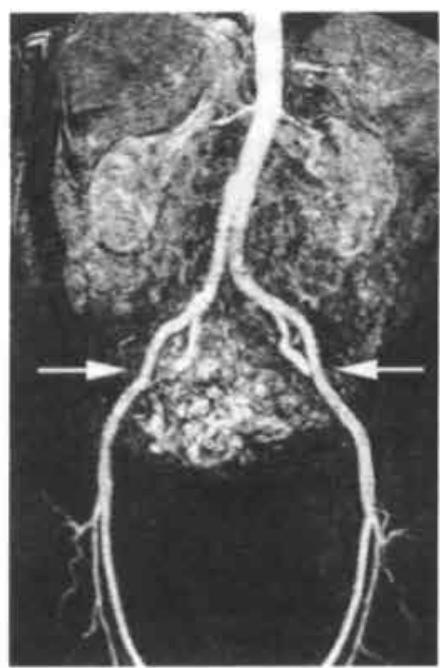

B

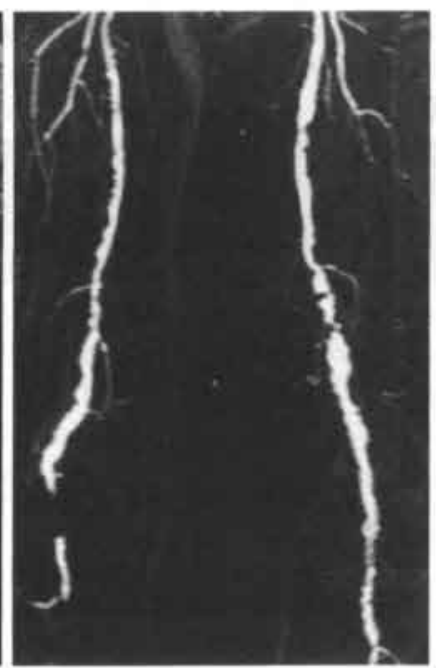

C

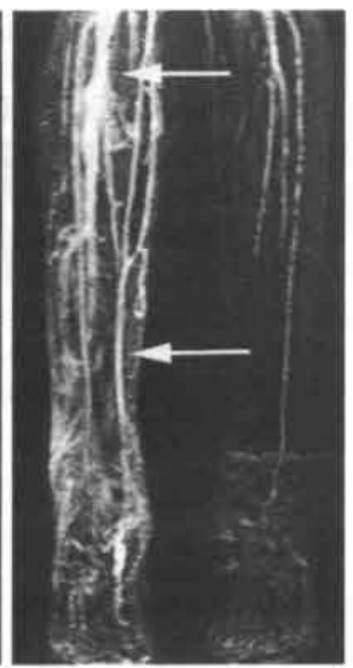

Figure 4. Images of different patients shows examples of vessel segments with different interpretability scores and venous enhancement. A: Suboptimal interpretability (arrows) because of too early acquisition of the pelvic station (mistiming the contrast material. B: Excellent interpretability of all upper leg vessel segments, despite severe stenotic and aneurysmatic pathology. C: Poor vessel segment interpretability (arrows) in the right lower leg because of severe venous overprojection.

In 80 patients ( $61 \%$ of all patients) interpretability of all vessel segments was good (SIS score $=0)$. Twenty-one patients ( $16 \%$ of all patients) had one or more vessel segments with a maximum SIS of 1 (suboptimal vessel segment interpretability; 6 in the pelvic and upper leg stations, 15 in the lower leg station) and 30 patients ( $23 \%$ of all patients) had one or more vessel segments that had a maximum SIS of 2 (poor vessel segment interpretability; 2 in the upper leg station, 28 in the lower leg station). Concerning venous enhancement, only $29 \%$ of all patients showed no venous enhancement at all. In $39 \%$ of all patients, at least one vessel segment had much 
venous enhancement next to it (of which $11 \%$ [of the 39\%] was next to a pelvic or upper leg vessel segment and $28 \%$ [of the $39 \%$ ] next to a lower leg vessel segment).

Concerning misregistration artifacts after subtraction and the possible effects on the SIS per station we found 17 stations with misregistration artifacts (10 patients). In 13 of these stations, all vessel segments had a SIS of 0 . In the remaining four stations (4 different patients), two had a maximum SIS of 1 and two had a maximum SIS of 2 (both of these latter two, the VES score was also 2).

As the number of vessel segments with poor interpretability in pelvic and upper leg stations were very small ( 0 and $1 \%$, respectively) we only evaluated possible related factors that affected vessel segment interpretability for the lower leg station by taking the highest score of the SIS per lower leg as dependent variable in the multi-variate logistic regression analysis. Diagram 1, demonstrates the effects on image interpretability of the different covariables.

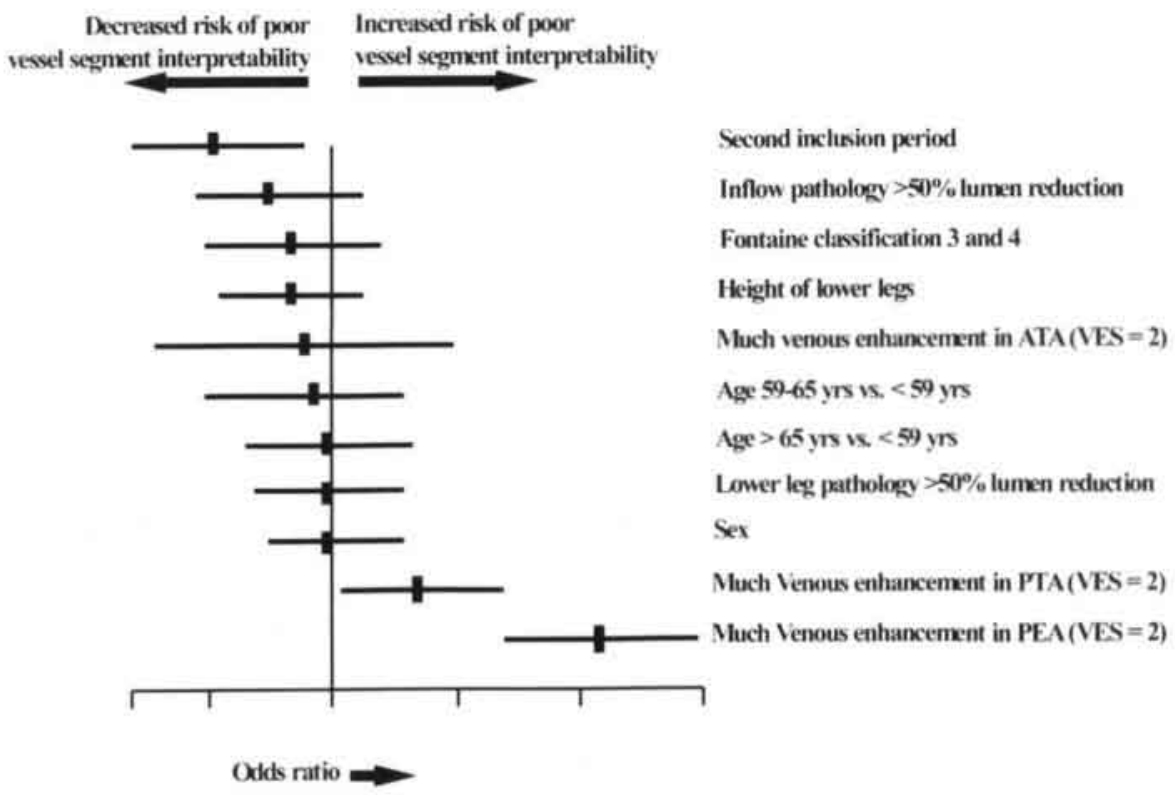

Diagram 1. Odds ratios for the different independent variables (listed in the diagram), predicting decreased or increased risk of poor vessel segment interpretability in the lower leg vessel segments. ATA: anterior tibial artery, PTA: posterior tibial artery, PEA: peroneal artery. VES: venous enhancement score. Vertical stripe: odds ratio for the particular covariable, horizontal stripe: confidence interval $(95 \%)$ of the odds ratio.

Table 2 tabulates the calculated SNR's and CNR's as found in the two groups of each 20 patients. Using the linear regression model, we found a statistically significant difference between SNR and CNR of the upper leg regions for patients from the first and second inclusion period, and for SNR and CNR between upper leg (superficial femoral and popliteal arteries) and lower leg station (peroneal, anterior and posterior tibial arteries). 
Table 2. Mean signal-to-noise and contrast-to-noise ratios as calculated for the vessel segments of the upper and lower leg station tabulated per inclusion period.

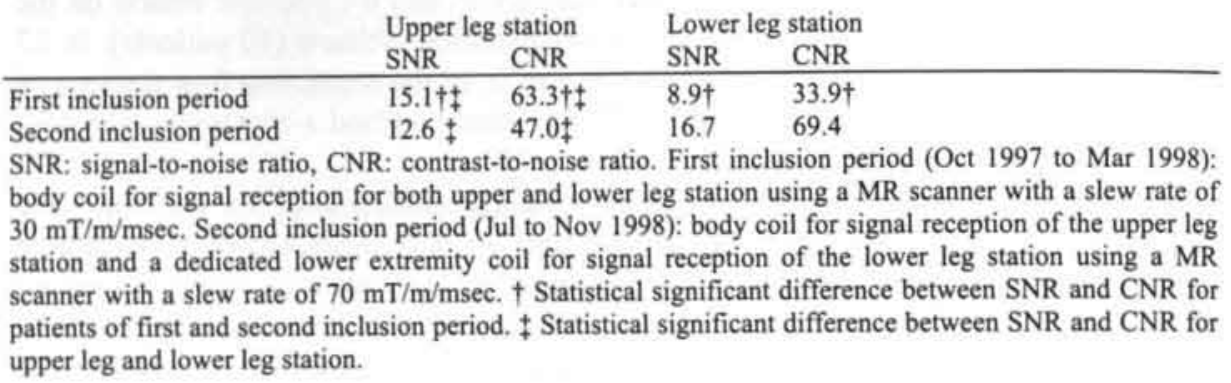

\section{Discussion}

Moving-Bed Infusion-tracking (MoBI-track) MR angiography can be regarded as a three-dimensional MR registration of only one single protracted bolus of gadolinium flowing through the peripheral vascular system. The MR contrast medium is injected intravenously and followed using a stepping MR table. One of the potential drawbacks of this technique is that infusion of gadolinium over a longer period $(>110$ seconds) may decrease vessel-to-background contrast because of uptake in surrounding tissues and venous enhancement. Moreover, subtraction of mask from contrast volumes can cause misalignment due to patient movement. With this study we have tried to get insight in frequency and cause of phenomena which may influence image interpretability.

In $94.5 \%$ of all vessel segments, image interpretability was assessed as good and in only $2.5 \%$ of all vessel segments, image interpretability was poor. With the multivariate logistic regression analysis we demonstrated that venous enhancement was the only major covariable related to impaired image interpretability. However, venous enhancement does not always decrease vessel segment interpretability. In the pelvic station no poor vessel segment interpretability was found despite the fact that in $9 \%$ of all pelvic vessel segments venous enhancement was found. The $3 \%$ of pelvic vessel segments with suboptimal vessel segment interpretability (SIS of 1 ) were due to mistiming of the bolus of contrast material (too early) because of the fixed timing delay. In $25 \%$ of upper leg vessel segments and $10 \%$ of lower leg vessel segments, venous enhancement was visible.

An interesting result was the fact that neither the clinical severity of symptoms (Fontaine classification) nor the presence of obstructive lesions (VPS) had any serious effect on image interpretability. However, presence of obstructive lesions was only based on MR angiographic data since not all patients underwent X-ray angiography as the standard of reference.

One of our hypotheses for preliminary venous enhancement in the lower legs, was the height of the lower leg arteries as compared to the level of inflow arteries. It was our assumption that a gravity effect (lower leg arteries lower than the inflow arteries) could worsen venous enhancement and therefore disturb image interpretability. However, no statistically significant influence could be demonstrated. 
The use of the dedicated lower extremity coil and more sophisticated equipment decreased the risk of poor vessel segment interpretability statistical significantly (factor 10 on average; diagram 1). However, if venous enhancement in the lower legs was excluded as a covariable in the multi-variate analysis, this decreased risk on poor vessel segment interpretability as shown in diagram 1 is not statistically significant anymore. This might be explained by the fact that the use of the coil improves arterial interpretability predominantly in the presence of venous enhancement or that the coil induces venous enhancement. However, no statistically significance relationship was found between venous enhancement and the use of the lower extremity coil (using a separate logistic regression analysis).

A final remark on the covariables should be made concerning the very high odds ratio of venous enhancement in the peroneal arteries. If the crude odds ratio was calculated (this covariable as the single independent covariable), the figure was still very high; 120 , demonstrating the tremendous increased risk of poor interpretability if venous enhancement occurs.

Due to the scanner update, our patient population was split into two consecutive groups of patients, who did not differ statistically significantly on the basis of patient characteristics. However, statistically significant differences were found in SNR's and CNR's as shown in table 2. The SNR's and CNR's of the upper leg arteries of patients examined during the second inclusion period were found to be statistically significantly lower. This is probably due to shorter TR and TE values as well as smaller voxel size used during the second inclusion period. As SNR and CNR of the lower leg arteries were relatively low (statistically significant as compared to the upper leg arteries) for patients of the first inclusion period, we started using a dedicated lower extremity coil to improve these figures. Because of the use of the dedicated lower extremity coil, the SNR's and CNR's doubled, despite shorter TR and $\mathrm{TE}$ and smaller voxel size. Although the subjective interpretability was unaffected by the use of this coil, we think that the increased SNR and CNR are valuable, especially when more optimized protocols per station can be used.

As this dedicated lower extremity coil is a prototype, we were able to implement our foot immobilizer (figure 2). This foot immobilizer was designed to strap down the patient securely and comfortably (slight exorotation of the feet and flexion in the knees) thereby diminishing the risk of misregistration after subtraction. Despite the use of the foot immobilizer, misregistration artifacts occurred in 17 stations. However, in only 4 out of 17 stations with misregistration, suboptimal or poor vessel segment interpretability occurred. If the nonsubtracted original slices were evaluated, no motion artifacts were present and all data could be evaluated perfectly, except for venous enhancement in the 2 stations with poor vessel segment interpretability (SIS of 2).

The implemented features by Philips medical systems (semi-automatic table movement and angulation per stack) combined with the use of a fixed timing delay (thereby eliminating the need for a timing sequence), allowed us to cut down on total patient handling time to about 15 to 20 minutes. 


\section{Improvements}

As venous enhancement particularly in the lower legs seemed to be the major contributor to poor vessel segment interpretability we also used the multi variate logistic regression analysis with venous enhancement of the lower legs as dependent variable, to determine any influences of the following covariables: inclusion period, inflow pathology, Fontaine classification, height of the lower legs, age, lower leg pathology per artery and sex. Only pathology in the peroneal arteries increased the risk of venous enhancement in the lower legs statistically significantly. Therefore, we think that shortening the time between the start of contrast material injection and acquisition of the lower leg station is the sole solution to lowering poor vessel segment interpretability.

Using the newest features on our scanner we are now able to decrease the time between injection of contrast material and acquisition of the middle of K-space of the lower leg station to approximately 100 seconds by using a realtime bolus arrival detection system (Bolustrak, Philips Medical Systems, Best, the Netherlands), and station optimized sequences. Figure 5 shows the first results of this further optimized MoBI-track technique as compared to the technique used for this study in a healthy volunteer.
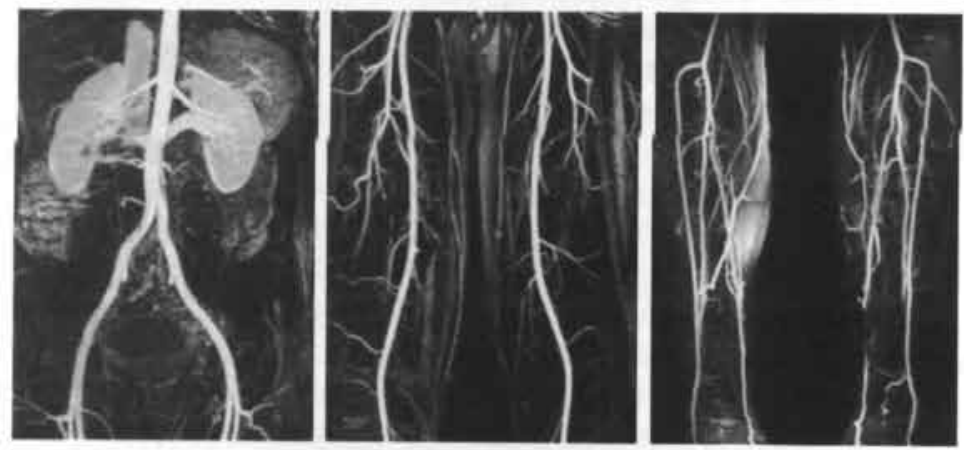

A
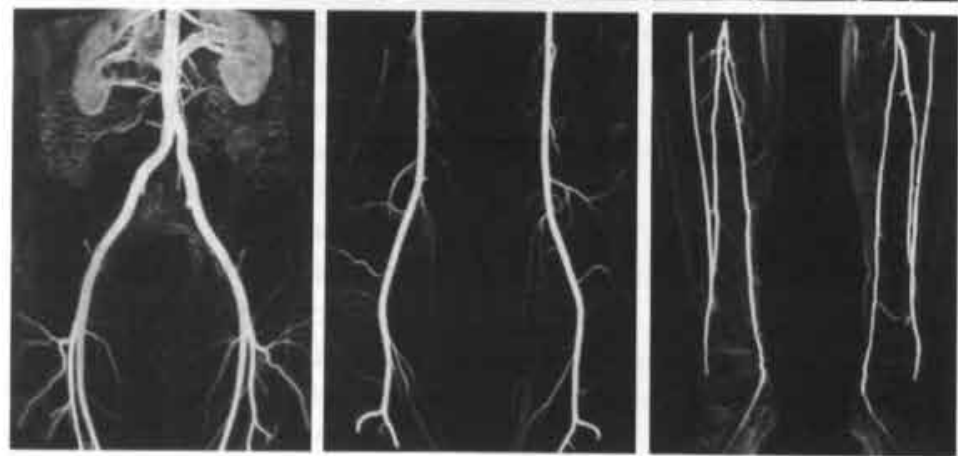

B

Figure 5. Coronal maximum intensity projection images of the standard Moving-Bed Infusion-tracking (MoBI-track) technique and with the station-optimized MoBI-track technique in a 25-year-old healthy volunteer. A: Although good delineation of the arterial system is obtained, venous enhancement particularly in the lower leg station can be seen. B: With the optimized MoBI-track technique, better vessel-to-background contrast and images without any venous enhancement can be obtained. 


\section{Conclusion}

Good interpretability of contrast-enhanced peripheral MR angiograms using the MoBI-track technique was demonstrated in the majority of patients and vessel segments. Major contributor to decreased image interpretability is venous enhancement, particularly in the lower leg station. Faster acquisition techniques and better bolus timing can further improve image interpretability. 


\section{References}

1. Wang Y, Lee HM, Khilnani NM, Trost DW, Jagust MB, Winchester PA, Bush HL, Sos TA, Sostman HD (1998) Bolus-chase MR digital subtraction angiography in the lower extremity. Radiology 207: 263-269.

2. Rofsky NM, Johnson G, Adelman MA, Rosen RJ, Krinsky GA, Weinreb JC (1997) Peripheral vascular disease evaluated with reduced-dose gadolinium-enhanced MR angiography. Radiology 205: 163-169.

3. Watanabe Y, Dohke M, Okumura A, Amoh Y, Ishimori T, Oda K, Dodo Y (1998) Dynamic subtraction MR angiography: first-pass imaging of the main arteries of the lower body. AJR Am J Roentgenol 170: 357-360.

4. Ho KYJAM, Leiner T, de Haan MW, Kessels AGH, Kitslaar PJEHM, van Engelshoven JMA (1998) Peripheral vascular tree stenoses: evaluation with moving-bed infusiontracking MR angiography. Radiology 206: 683-692.

5. Banga J.D., Stoffers H.E.J.H., Berengoltz-Zlochin S.. Kliniek, epidemiologie en prognose. Hartbulletin $\mathrm{Nr}$ 6; dec 1997: 185-189.

6. Kouwenhoven M., Fuderer M, Grootes P., Ho K.Y.J.A.M., van Engelshoven J.M.A. MRA Optimized Non-Uniformity Correction for Rigid Synergy Coils. Proceedings of the seventh scientific meeting and exhibition of the ISMRM. 


\title{
Chapter 7
}

\section{Peripheral Vascular Tree Stenoses: Evaluation with Bloodpool-enhanced and Gd-DTPA- enhanced MR Angiography}

\author{
Submitted
}

Authors: Ho K.Y.J.A.M., Leiner T, de Haan M.W., Kessels A.G.H., van Engelshoven J.M.A. 



\begin{abstract}
Purpose: To evaluate the feasibility of bloodpool-enhanced peripheral MR angiography using iron-oxide particles and to compare the results with Gd-DTPAenhanced peripheral MR angiography and diagnostic X-ray angiography as the standard of reference.

Subjects and Methods: Ten patients, 7 men and 3 women, underwent both gadolinium-enhanced and bloodpool-enhanced (Omniscan and NC100150, respectively, Nycomed Amersham, Oslo, Norway) MR angiography. Gadoliniumenhanced MR angiography was done with the moving-bed infusion-tracking technique. Bloodpool-enhanced MR angiography consisted of both first pass and steady state imaging. All data of both the gadolinium-enhanced and steady state bloodpool-enhanced MR angiography, were evaluated subjectively (vessel visibility score; good or poor) and objectively (shaded surface rendering and SNR/CNR calculations) for image quality. Furthermore, both gadolinium-enhanced and steady state bloodpool-enhanced MR angiographic data were evaluated for detection and grading of hemodynamically significant stenoses (lumen reduction $250 \%$ ) using Xray angiography as the standard of reference.
\end{abstract}

Results: No severe side effects were noted in any of the patients for any of the examinations. First pass bloodpool-enhanced MR angiography was successful in 8 out 10 patients. Subjectively, poor vessel visibility was seen in 3 out of 270 vessel segments and shaded surface rendering succeeded in 29 out of 30 stations using gadolinium-enhanced MR angiography. Using steady-state bloodpool-enhanced MR angiography 17 out of 270 vessel segment were poorly visible and 13 out of 30 stations succeeded using shaded surface rendering. Compared to steady-state bloodpool-enhanced MR angiography, SNR's and CNR's were statistically significantly higher for gadolinium-enhanced MR angiography in the pelvic and upper leg arteries, but lower in the lower leg arteries. Sensitivity and specificity for detecting and grading hemodynamically significant stenoses were $92 \%$ and $98 \%$, respectively for gadolinium-enhanced MR angiography and $71 \%$ and $94 \%$, respectively for steady-state bloodpool-enhanced MR angiography. The low sensitivity figure for bloodpool-enhanced MR angiography was mainly due to erroneous interpretation in the lower legs.

Conclusion: Depiction of peripheral vascular pathology using ultrahigh resolution bloodpool-enhanced MR angiographic data is feasible. Results in the lower leg region were suboptimal. However, with more optimized sequences bloodpool-enhanced MR angiography of the lower extremities using $\mathrm{NC100150}$ can be a valuable asset to peripheral MR angiography. 


\section{Introduction}

Gadolinium-enhanced peripheral MR angiography has proven its efficacy as an arterial imaging modality [1-4]. However, due to the limited imaging window between arterial and venous enhancement, a trade-off between temporal and spatial resolution has to be made. Contrast-enhanced acquisitions are aimed at imaging during bolus passage for high enough intra-arterial concentration of contrast material (sufficient arterial signal), and imaging before tissue or venous enhancement occurs. Therefore, despite the ultrafast scanner hardware available today, voxel size typically is in the order of 5 to $10-\mathrm{mm} 3$. To overcome these limitations, bloodpool agents (BP) can be used. These contrast agents do not leak into surrounding tissue, have a prolonged half life (hours instead of minutes) and have a stronger T1 reducing effect than gadolinium-chelates, eliminating the need for bolus imaging (imaging during steady state gives sufficient arterial enhancement). Therefore longer acquisition strategies, acquiring high spatial resolution volumes (voxel volumes of $\leq 1-\mathrm{mm}^{3}$ ), can be applied. High spatial resolution acquisition strategies using BP-enhanced MR angiography not only is an advantage but also a necessity, since arteries and veins are enhanced equally, making it difficult separating the two. Separation of arteries from veins can only be done if sufficient spatial resolution allows strict differentiation between arteries and veins.

As part of a phase two safety and efficacy study, it was our purpose to evaluate the feasibility of BP-enhanced MR angiography using a single intravenous injection of NC 100150, which is a starch-coated iron particle bloodpool agent (Nycomed Amersham, Oslo, Norway) for imaging the peripheral arteries in 10 patients and to compare the results with gadolinium-enhanced peripheral MR angiography and diagnostic X-ray angiography as the standard of reference.

\section{Methods and Subjects}

\section{MR Angiography}

All MR angiographic scans were obtained with a 1.5 T MR system (Gyroscan New Technology, release 5.1 and 6.1; Philips Medical Systems, Best, The Netherlands). The first two patients were examined on a system with a maximum gradient-strength of $15 \mathrm{mT} / \mathrm{m}$ and a risetime of $0.5 \mathrm{~ms}$. The other eight patients were examined on a system with a maximum gradient-strength of $21 \mathrm{mT} / \mathrm{m}$ and a risetime of $0.3 \mathrm{~ms}$. The standard quadrature body coil was used for signal transmission and reception imaging the pelvic and upper leg station and a dedicated prototype quadrature phased array lower extremity coil [5] was used for signal reception of the lower leg station. The procedure consisted of three parts. Part one: patient selection. Part two: BP-enhanced MR angiography during injection of contrast material (first pass). Part three: high resolution steady-state BP-enhanced (HR-BP) MR angiography. 


\section{Part One}

Ten patients were selected on the basis of the following criteria: patients could be either male or postmenopauzal female, and had complaints of intermittent claudication objectified with Duplex ultrasonography. If a hemodynamically significant stenosis (lumen reduction $250 \%$ ) was detected with Duplex ultrasonography, patients were invited to undergo gadolinium-enhanced peripheral MR angiography using an optimized form of the recently described MoBI-track technique (Gd-MoBI-track) demonstrated in figure 1 [6].

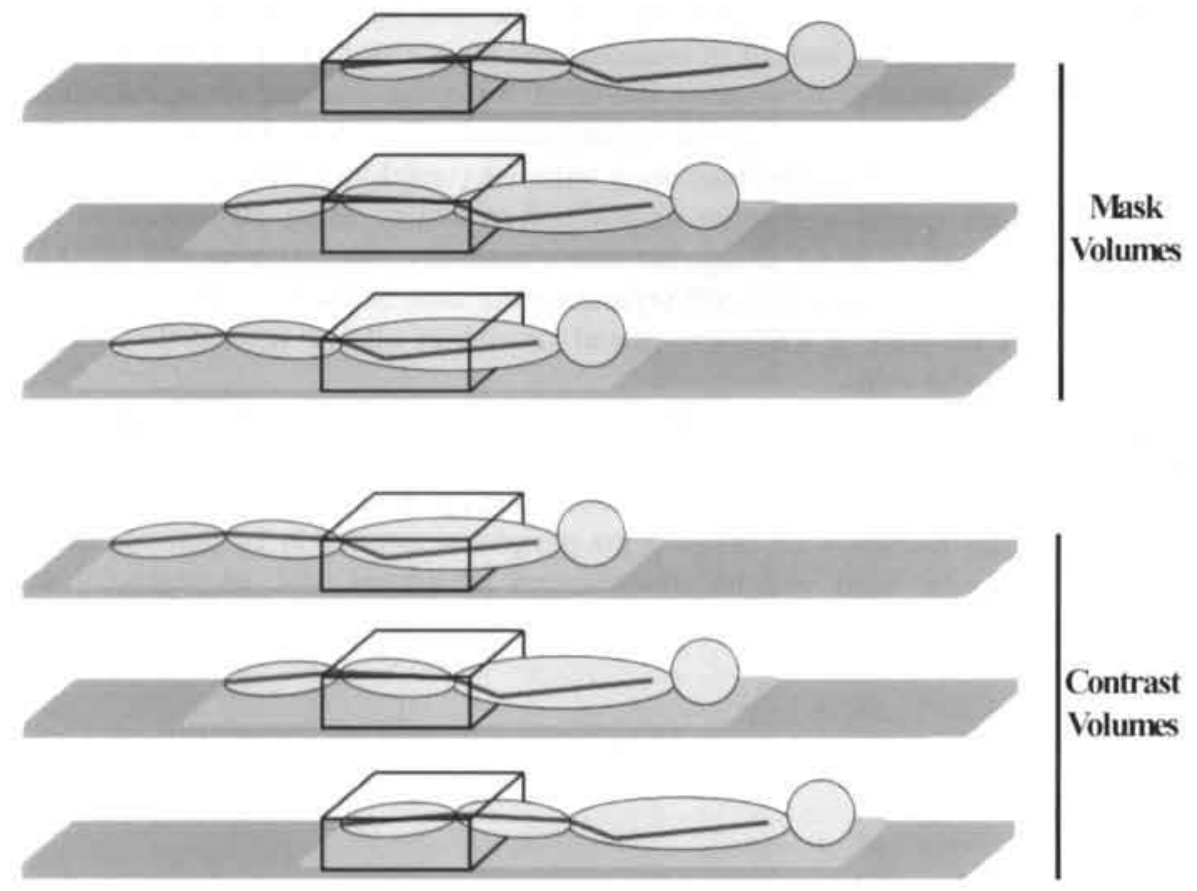

Figure 1. Schematic illustration of the principal of the Moving-Bed Infusion-tracking (MoBI-track) technique. The sequence of table movements is shown for unenhanced (mask volumes) imaging (top) and gadolinium-enhanced imaging (bottom). The table is moved automatically by the scanner software.

With this technique the entire peripheral vasculature (pelvic, upper leg and lower leg stations) is imaged during the infusion of only one single bolus of contrast material. Imaging parameters for the MoBI-track sequence were: 7.0/2.0/30/450/512 (TR/TE/FA/field-of-view/matrix). If the hemodynamically significant stenosis found on Duplex ultrasonography was confirmed with Gd-MoBI-track MR angiography, patients were invited to participate in the BP-enhanced MR angiographic study. Written informed consent was given by all invited patients for both Gd-MoBI-track MR angiography and BP-enhanced MR angiography. The entire study protocol was approved by the medical ethics committee of our hospital. 


\section{Part Two}

All patients underwent first pass bloodpool-enhanced MR angiography. Three of them underwent timing sequences with the objective to determine the length of the arterial-venous window. Imaging parameters for these sequences were: 4.5/1.2/30/450/256 (TR/TE/FA/field-of-view/matrix). Injection rates for these sequences varied from 1-2-mL/sec using a MR compatible remote controlled power injector (Spectris MR injector, MEDRAD, Pittsburgh, PA). All others underwent first pass bloodpool-enhanced MR angiography using the MoBI-track technique (FP-BP MoBI-track). For the FP-BP MoBI-track techniques, injection rates varied from 0.2$\mathrm{mL} / \mathrm{sec}$ (first patient) to $0.3-0.4-\mathrm{mL} / \mathrm{sec}$ (all other patients). Table 1 tabulates the different MR angiographic techniques used for the different patients.

Table 1. MR Angiographic techniques performed per Patient.

\begin{tabular}{llll} 
& Gd-MoBI & FP-BP & HR-BP \\
\hline Patient no. 1 & $V$ & Pelvic/upper/lower & $\checkmark$ \\
Patient no. 2 & $\checkmark$ & Timing & $\checkmark$ \\
Patient no. 3 & $\checkmark$ & Timing & $\checkmark$ \\
Patient no. 4 & $V$ & Timing & $\checkmark$ \\
Patient no. 5 & $\checkmark$ & Pelvic & $\checkmark$ \\
Patient no. 6 & $V$ & Pelvic/upper & $\checkmark$ \\
Patient no. 7 & $\checkmark$ & Pelvic/upper & $\checkmark$ \\
Patient no. 8 & $V$ & Pelvic/upper/lower & $\checkmark$ \\
Patient no. 9 & $\checkmark$ & Pelvic/upper/lower & $\checkmark$ \\
Patient no. 10 & $\checkmark$ & Pelvic/upper/lower & $\checkmark$
\end{tabular}

$\checkmark$ : performed. Gd-MoBI: gadolinium-enhanced MR angiography using the Moving-Bed Infusion-tracking technique. FP-BP: first pass bloodpool enhanced MR angiography with the same sequence as used with the Gd-MoBI technique, except for the three timing sequences; Pelvic: of the pelvic region; Pelvic/upper: of the pelvic and upper leg region; Pelvic/upper/lower: of the pelvic, upper leg and lower leg region. HR-BP: high resolution steady state bloodpool-enhanced MR angiography.

\section{Part Three}

The basic sequence for HR-BP MR angiography was a coronal three-dimensional gradient-recalled echo technique. TR and TE varied slightly depending on the different maximum gradient strengths for the MR systems, spatial resolution and use of the dedicated lower extremity coil. TR and TE for the pelvic and upper leg region typically were 6.1 and $1.7-\mathrm{msec}$, respectively. Flip angle was $30^{\circ}$. Field of view for the pelvic and upper leg region varied slightly from 450 (release 5.1 .2 of scanner software) to $512-\mathrm{mm}$ (release 6.1.1 of scanner software). FOV for the lower leg region was $384-\mathrm{mm}$. Matrix size for all three stations was $512 \times 512$. Slice thickness for the pelvic and upper leg region was $1-\mathrm{mm}$ and for the lower leg region $0.75-\mathrm{mm}$. Therefore, voxel volume for the pelvic and upper leg region varied from $0.81-\mathrm{mm}^{3}$ to $1-\mathrm{mm}^{3}$, except for the first patient, where an isotropic voxel volume of $0.42-\mathrm{mm}^{3}$ was used for all three stations. For the lower leg region an isotropic voxel volume of 0.42 $\mathrm{mm}^{3}$ was used in all patients. 


\section{X-ray Angiography}

In all patients except one (X-ray angiographic exam canceled due to anxiety of patient), diagnostic X-ray angiography (both conventional angiography and digital subtraction angiography [DSA]) was performed within one day of MR angiography with an Integris 5000 angiosuite (Philips Medical Systems, Best, The Netherlands) and a power injector (MEDRAD Inc., Pittsburgh, PA). All angiographic procedures were supervised by an experienced vascular radiologist. In 7 patients, the diagnostic $\mathrm{X}$-ray angiographic procedure consisted of puncturing the common femoral artery, placing a 5-F catheter in the distal aorta just above the bifurcation to examine both legs. In 2 patients, the 5-F catheter was placed in the external iliac artery to examine only the leg, on the side the patient had complaints. A nonionic contrast medium (Omnipaque $^{8}$, Nycomed, Cork, Ireland) was used in variable volumes and flow rates depending on both the location of the tip of the catheter and the procedure used (conventional angiography or DSA). Cut films were obtained in the posteroanterior direction for conventional angiography, and in all patients additional posteroanterior and oblique views were obtained using digital subtraction angiography.

\section{Image Evaluation and Interpretation}

For evaluation purposes, the arterial tree was divided into the following segments: aorta, both left and right common iliac arteries, external iliac arteries, common femoral arteries, deep femoral arteries, upper and lower parts of the superficial femoral arteries, popliteal arteries, upper and lower parts of the anterior tibial, posterior tibial and peroneal arteries. Signal-to-noise (SNR), and contrast-to-noise (CNR) ratio's were calculated for each of these vessel segments provided there was a nondiseased part of the vessel segment. For the FP-BP MoBI-track technique SNR and CNR were only calculated for the pelvic region. SNR was defined as signal intensity in the vessel segment divided by the standard deviation of the signal intensity of noise (measured in air). CNR was defined as the signal intensity in the vessel segment minus the signal intensity directly next to that vessel segment, divided by the standard deviation of the signal intensity of noise. Vessel visibility was evaluated subjectively by determining the ability to distinguish the arterial vessel segments from other arteries or veins. A two-point scoring scale was used, ranging from score 0 , if the vessel segment was interpretable in its entirety and was distinguishable from its neighboring vessels, or score 1 if the vessel segment was not distinguishable. To (semi-) objectively evaluate ease of arterial-venous separation, shaded surface rendered volumes were made by using different thresholds and inclusion points on whole volumes. The aim of the shaded surface rendering test was to separate arteries from veins. This was done for all available MR angiographic techniques (Gd-MoBI-track, FP-BP MoBI-track and HR-BP MR angiography).

For each patient, degree of stenoses were assessed by two observers in consensus and categorized using a scoring-scale from 0 to 3 , where $0=$ no abnormality or stenosis with a lumen reduction $<50 \%, 1=$ lumen reduction ranging from $50 \%$ to $75 \%, 2$ = lumen reduction ranging from $75 \%$ to $100 \%$, and $3=$ complete occlusion. Only the most severe stenosis per vessel segment was taken into account. Stenoses 
were measured on Gd-MoBI-track and HR-BP-enhanced volumes and X-ray angiograms. All detecting and grading of stenoses was done on an EasyVision workstation (Philips Medical Systems, Best, the Netherlands), on the original slices and, with MR angiographic data, also on zoomed maximum intensity projection images with an electronic caliper with $0.1-\mathrm{mm}$ accuracy. Evaluating a technique, the observers were unaware of the results of other techniques because evaluation of the different techniques was performed 2 months apart.

\section{Statistical Analysis}

To evaluate any statistically significant differences between SNR and CNR calculations, the Mann-Whitney test was used. To evaluate any statistically significant differences between sensitivities and specificities measured on both GdMoBI-track and HR-BP MR angiography the McNemar symmetry test was used.

\section{Results}

No serious adverse events were observed in any patients, both for Gd-MoBI-track MR angiography and BP-enhanced MR angiography. In one patient a slight rise in creatinin levels was observed directly after the BP-enhanced MR angiographic exam. Values rose from $90 \mu \mathrm{mol} / 11$ hour pre-injection to $230 \mu \mathrm{mol} / 2$ hours post-injection of $\mathrm{NC100150}$ (normal values: $60-120 \mu \mathrm{mol} / \mathrm{l}$ ). Creatinin levels returned to normal within one day and no medical intervention was needed. Representative examples of the results of diagnostic X-ray angiography, Gd-MoBI-track and both BP-MoBI-track and HR-BP MR angiography are shown in figure 2.

All gadolinium-enhanced and bloodpool-enhanced exams were technically successful except for two of the three dynamic first pass BP-enhanced studies (patients 2 and 3 ). These two studies failed due to too low arterial enhancement using modulus subtraction and an injection rate of $1-\mathrm{mL} / \mathrm{sec}$ in patient number 2 and due to the fact that real and imaginary data could not be reconstructed to perform complex subtraction in patient number 3 [7]. The first pass dynamic timing scan that was successful, using an injection rate of $2-\mathrm{mL} / \mathrm{sec}$ demonstrated an arterial-venous window of $159 \mathrm{sec}$ between the first adequate arterial image (bolus arrival) and the last interpretable arterial image.

SNR, and CNR calculations are tabulated in table 2. The subjective visibility scoring scale showed that, 3 out of 270 vessel segments ( 2 different arteries) using Gd-MoBI-track MR angiography were uninterpretable. Using HR-BP MR angiography, 17 ( 15 of these were lower leg vessel segments in 10 different arteries) out of 270 vessel segments were uninterpretable. The ability to create arterial shaded surface rendered volumes is tabulated in table 3 . Stenoses found with X-ray angiography are tabulated in table 4 .

With X-ray angiography, 5 lower leg vessel segments were uninterpretable due to misalignment between mask and contrast images for DSA (in one patient). 
A

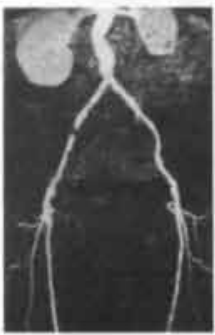

B
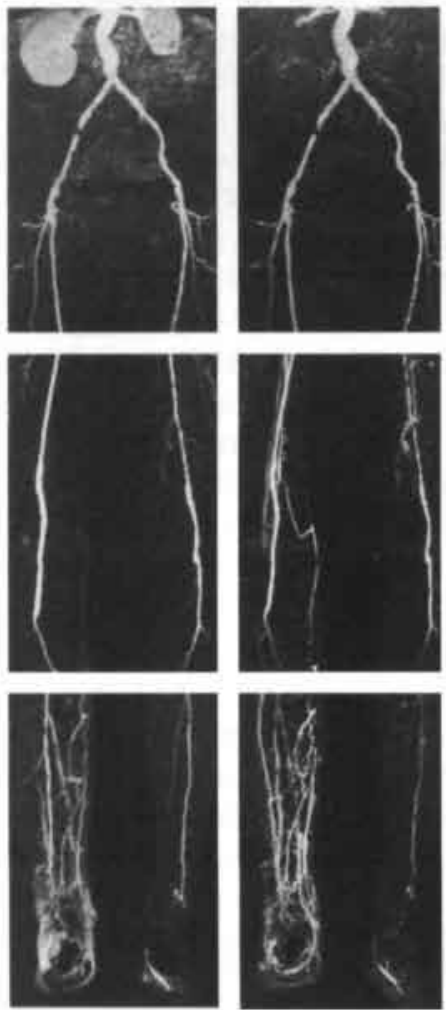

C

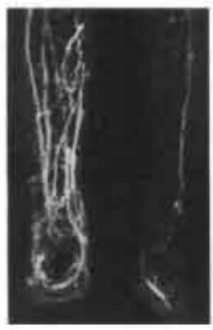

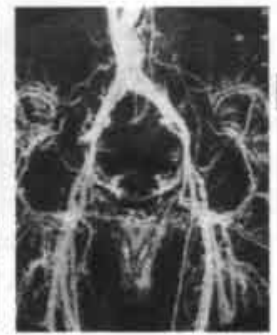

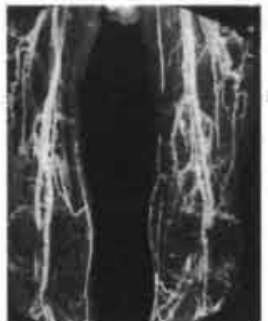

D
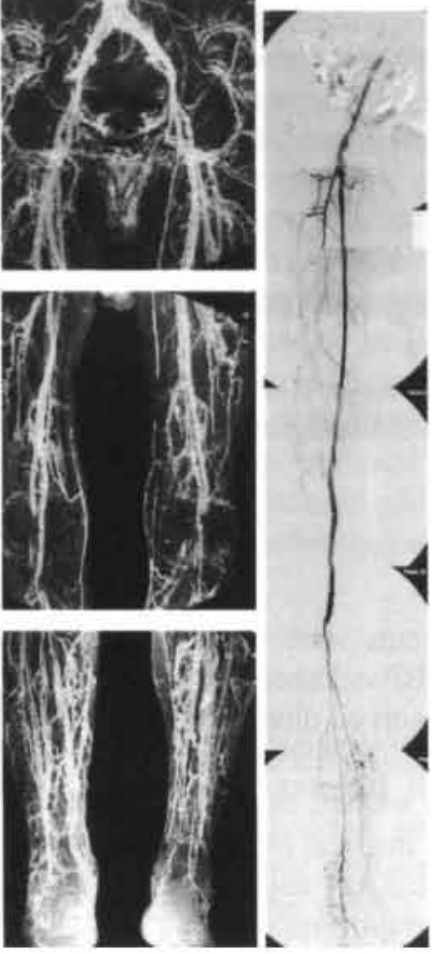

Figure 2. Representative examples of coronal maximum intensity projection images of a 66year-old man. A: Images obtained with gadolinium-enhanced MovingBed Infusion-tracking (MoBI-track) MR angiography. B: Images obtained with first pass $\mathrm{NC100150}$ (bloodpool agent) MoBI-track MR angiography. C: Images obtained with high resolution $\mathrm{NC100150-}$ enhanced MR angiography. D: Corresponding digital subtraction angiogram of the right leg.

\{Three-dimensional views of the high resolution bloodpoolenhanced data can be seen on the Image-CD $\}$.

Table 2. Signal-to-noise ratios (SNR) and contrast-to-noise ratios (CNR) calculations.

\begin{tabular}{llll} 
& $\begin{array}{l}\text { Gd-MoBI-track } \\
\text { SNR/CNR }\end{array}$ & $\begin{array}{l}\text { FP-BP MoBI-track } \\
\text { SNR/CNR }\end{array}$ & $\begin{array}{l}\text { BP-HR } \\
\text { SNR/CNR }\end{array}$ \\
\hline pelvic station & $23 / 22$ & $24 / 24$ & $20 / 16$ \\
upper leg station & $34 / 34$ & & $25 / 22 \dagger$ \\
lower leg station & $29 / 27$ & & $64 / 56 \dagger$
\end{tabular}

Gd-MoBI-track: gadolinium-enhanced MR angiography using the MoBI-track technique, FP-BP MoBItrack: Bloodpool first pass MR angiography using the MoBI-track technique, BP-HR: Bloodpool-enhanced high resolution MR angiography. † Statistically significant difference between Gd-MoBI-track and BP-HR SNR and CNR figures.

Table 3. Shaded surface display results.

\begin{tabular}{llll} 
& Gd-MoBI-track & FP-BP MoBI-track & BP-HR \\
\hline Pelvic station & 10 out of 10 & 7 out of 7 & 9 out of 10 \\
Upper leg station & 10 out of 10 & 2 out of 6 & 4 out 10 \\
Lower leg station & 9 out of 10 & 0 out of 3 & 0 out of 10
\end{tabular}

Note.- numbers represent the total number of volumes that successfully could be processed, separating arteries from veins. Gd-MoBl-track: Gadolinium-enhanced MR angiography using the MoBI-track technique. FP-BP MoBl-track: First pass bloodpool-enhanced MR angiography using the MoBI-track technique. BP-HR: Bloodpool-enhanced high resolution MR angiography. 
Table 4. Stenoses found on X-ray angiography.

\begin{tabular}{llllll}
\multicolumn{5}{c}{ Grade of stenosis } \\
& 0 & 1 & 2 & 3 & missing \\
\hline pelvic station & 59 & 2 & 3 & 4 & 22 \\
upper leg station & 35 & 3 & 1 & 9 & 12 \\
lower leg station & 75 & 2 & 0 & 14 & 29 \\
Grade of stenoses: 0 lumen narrowing & $<50 \%, 1: 50 \%$ to $75 \%$ lumen narrowing, $2: 75 \%$ to $100 \%$ lumen \\
narrowing, 3 complete occlusion.
\end{tabular}

Sensitivity and specificity for detecting and grading hemodynamically significant stenoses in all vessel segments (lumen reduction of $250 \%$ ) were $92 \%$ and $98 \%$, respectively, for Gd-MoBI-track MR angiography and $71 \%$ and $94 \%$, respectively, for steady state HR-BP MR angiography. If sensitivity and specificity were calculated excluding the lower leg station, figures were $100 \%$ and $98 \%$, respectively for GdMoBI-track and $95 \%$ and $95 \%$ for steady state high resolution BP-enhanced MR angiography, respectively. These latter figures do not differ statistically significantly between Gd-MoBI-track and high resolution BP-enhanced MR angiography.

\section{Discussion}

Ultrahigh resolution bloodpool-enhanced MR angiography of the peripheral arteries using NC100150 is feasible for the pelvic and upper leg arteries, but due to insufficient resolution suboptimal for the lower leg arteries. As we had only 10 patients included in this study, most results are merely an indication of their value. Even more so, as this was our first experience with NC100150, it is most likely that sequences used for both FP-MoBI-track as well as HR-BP MR angiography were suboptimal.

Despite these limitations, first pass imaging using bloodpool-enhanced MR angiography was successful for all imaged pelvic stations. As we had insufficient volume of bloodpool contrast material (12-mL on average), subsequent stations (upper leg and lower leg stations) were depicted suboptimally (figure 2). However, the succeeded timing sequence in patient no. 4 demonstrated, that provided sufficient volume of contrast material, a full bloodpool-enhanced MoBI-track examination is likely to succeed, as the arterial-venous window in the lower legs seems similar to that found when using gadolinium-chelates.

The SNR and CNR calculations showed significantly higher figures for Gd-MoBItrack than for HR-BP MR angiography for the pelvic and upper leg arteries, but significantly lower figures for the lower leg arteries. The higher figures for the pelvic and upper leg arteries using Gd-MoBI-track, might be explained by the high concentration of intra-arterial gadolinium-chelates during bolus passage, as well as the relatively large voxels used with this technique. Furthermore, for the pelvic station, the CNR figures are likely to be higher with the Gd-MoBI-track technique, as this station was acquired during breathhold, whereas with the HR-BP MR angiographic technique, no breathholding was applied. The relatively low figures for Gd-MoBI-track for the lower leg arteries can be explained by the dilution effect in this last station (leakage of the gadolinium-chelates from the capillaries), as well as 
by partial volume effects with the relatively large voxels used. On the other hand, SNR and CNR figures, using the HR-BP MR angiographic data were extremely high, despite the ultrahigh resolution of this data (voxel volume $0.42-\mathrm{mm}^{3}$ ). Therefore it is likely that resolution in this station can even further be increased.

The vessel visibility test was used to subjectively evaluate ease of arterial segmentation. Remarkable finding was, that both the pelvic as well as the upper leg arteries using HR-BP MR angiography, were easily distinguished from surrounding structures, including veins. However, using HR-BP MR angiography in the lower leg station, it can be very difficult to differentiate arteries from veins $(28 \%$ of lower leg arterial vessel segments could not be separated with the vessel visibility test). This finding was reflected in the results from the shaded surface rendering test, which was meant to evaluate the possibilities for (semi-) automated arterial-venous separation. None of the lower leg arteries imaged with HR-BP MR angiography could be separated with this tool, while with the Gd-MoBI-track technique, in 9 out 10 patients lower leg arteries could be separated. For the upper leg arteries, there was a discrepancy between the good subjective visibility scores using HR-BP MR angiography and the small number of arteries that could be separated using the shaded surface rendered technique. This might be explained by the fact that with shaded surface rendering, the arterial vascular tree of the upper legs is to be free of any neighboring veins over its entire length in order to have good results with shaded surface rendering, while as for the visibility score, the arterial tree is evaluated per vessel segments and close proximity of neighboring veins can be ignored.

For evaluation of disease, we used two observers experienced in evaluating MR angiographic data. Observers had to reach consensus on their evaluation. This was done to skip the learning curve for interpreting HR-BP data as much as possible, as we had only few patients included. Compared to Gd-MoBI-track MR angiography, sensitivity and specificity for detecting and grading hemodynamically significant stenoses (lumen reduction 250\%) were suboptimal when evaluating HR-BP MR angiographic data $(71 \%$ and $93 \%$, respectively). Especially the low figure for sensitivity was caused by misinterpretation of disease in the lower leg arteries. If sensitivity and specificity for detecting and grading hemodynamically significant stenoses was calculated for only the pelvic and upper leg arteries, values rose to $95 \%$ and $95 \%$, respectively, demonstrating the profound effect of misinterpretation in the lower leg arteries. However, as demonstrated by figure 3, delineation of the arteries with HR-BP MR angiography is superior to Gd-MoBI-track and seems comparable with X-ray angiography (DSA).

\section{Future Directions}

As demonstrated by this study, first pass data of the peripheral vasculature can be obtained using NC100150. This can be beneficial to bloodpool-enhanced MR angiography for three reasons: firstly, gross pathology is seen straight away on first pass data so no time has to be lost on acquiring or even evaluating HR-BP data containing no pathology. Secondly, as first pass data can be targeted very easily, this information (target of the arterial tree) can be used to separate the arterial tree from 
A

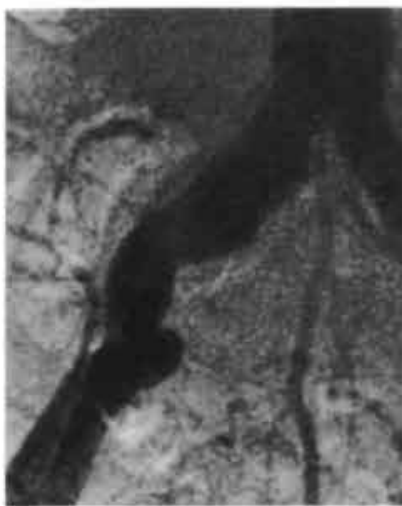

B

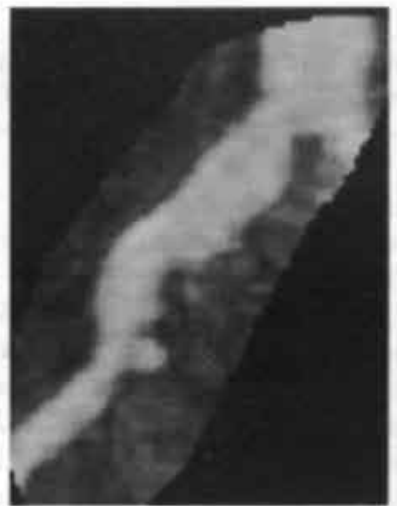

C

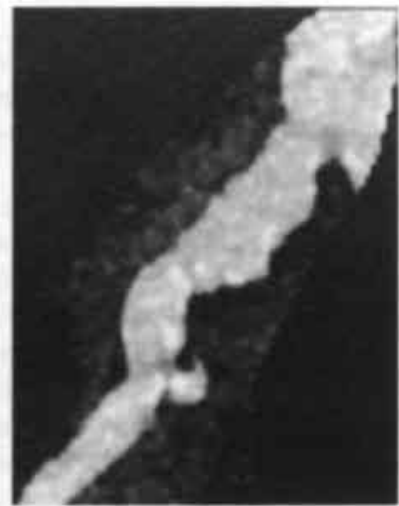

Figure 3. Vascular delineation with different techniques. A: X-ray angiography. B: gadolinium-enhanced MR angiography, C: high resolution bloodpool-enhanced MR angiography. Note the excellent delineation of the artery with this latter technique (in any desired obliquity) as compared to X-ray angiography.

surrounding structures in HR-BP data. Thirdly, first pass data can be used to selectively target pathology and evaluate only that part of an acquired HR-BP data set, decreasing time needed to evaluate the entire HR-BP data set, and decreasing the total amount of data that has to be handled.

\section{Conclusion}

Depiction of peripheral vascular pathology using ultrahigh resolution bloodpoolenhanced MR angiographic data is feasible. As these were our first experiments with NC100150, results in the lower leg region were suboptimal. However, with more optimized sequences and the use of first pass data to better separate arteries from veins, it is our believe that bloodpool-enhanced MR angiography of the lower extremities using $\mathrm{NC100150}$ can be a valuable asset to peripheral MR angiography. 


\section{References}

1. Prince MR. (1994) Gadolinium-enhanced MR aortography. Radiology 191: 155-164.

2. Leung DA, McKinnon GC, Davis CP, Pfammatter T, Krestin GP, Debatin JF (1996) Breath-hold, contrast-enhanced, three-dimensional MR angiography, Radiology 201: 569-571.

3. Ho KYJAM, de Haan MW, Kessels AGH, Kitslaar PJEHM, van Engelshoven JMA (1998) Peripheral vascular tree stenoses: detection with subtracted and nonsubtracted MR angiography. Radiology 206: 673-681.

4. Hany TF, Debatin JF, Leung DA, Pfammatter T (1997) Evaluation of the aortoiliac and renal arteries: comparison of breath-hold, contrast-enhanced, three-dimensional MR angiography with conventional catheter angiography. Radiology 204: 357-362.

5. Kouwenhoven M., Fuderer M, Grootes P., Ho K.Y.J.A.M., van Engelshoven J.M.A. MRA Optimized Non-Uniformity Correction for Rigid Synergy Coils. Proceedings of the seventh scientific meeting and exhibition of the ISMRM.

6. Ho KYJAM, Leiner T, de Haan MW, Kessels AGH, Kitslaar PJEHM, van Engelshoven JMA (1998) Peripheral vascular tree stenoses: evaluation with movingbed infusion-tracking MR angiography. Radiology 206: 683-692.

7. Wang Y, Lee HM, Khilnani NM, Trost DW, Jagust MB, Winchester PA, Bush HL, Sos TA, Sostman HD (1998) Bolus-chase MR digital subtraction angiography in the lower extremity. Radiology 207: 263-269.

8. Lee HM, Wang Y (1998) Dynamic k-space filling for bolus chase 3D MR digital subtraction angiography. Magn Reson Med 40: 99-104. 
Chapter 7 


\section{Chapter 8}

\section{MR Angiography: Runoff Vessels}

Published in European Radiology 1999; 7:1285-1289

as manuscript for the ECR 2000 Categorical Course "MRI - from Basic Knowledge to Advanced Strategies"

Authors: Ho K.Y.J.A.M., Leiner T., van Engelshoven J.M.A. 



\begin{abstract}
MR angiography has taken a huge step forward since the introduction of contrastenhanced MR angiography using gadolinium-chelates. The more conventional MR angiographic techniques like time-of-flight and phase contrast MR angiography have been ousted by contrast-enhanced MR angiography in most vascular areas. However, imaging the lower extremities, the major obstacle is the length of the vascular tree. In order to cover the entire peripheral vasculature, at least two to three field of views are required. Using contrast-enhanced MR angiography, best results are obtained if the vessels of interest are imaged during passage of a bolus of contrast material. Vesselto-background contrast in subsequent acquisitions using subsequent injections of contrast material is hampered by recirculation and leakage of previously injected gadolinium, enhancing both the venous system and surrounding tissue. To overcome this problem several research groups came up with different solutions. The main three strategies employed can be classified as either bolus catch, bolus chase or bolus track techniques.

The purpose of this article is to explain working mechanisms of the three bolus imaging strategies for imaging both inflow and outflow vessels of the lower extremities, to show their advantages and disadvantages, and to review results described in literature in imaging patients using these techniques.
\end{abstract}


Chapter 8 


\section{Introduction}

Gd-DTPA enhanced MR angiography (GdE MR angiography) received a lot of attention over the past few years as it increased image quality of MR angiography dramatically. Gd-DTPA (gadolinium-chelates) decreases T1 values of blood and when using a heavily T1-weighted sequence, saturating surrounding tissue, excellent vessel-to-background contrast can be obtained. Therefore the more traditional MR angiographic techniques like phase-contrast MR angiography and time-of-flight MR angiography have been ousted by GdE MR angiography in most vascular regions. However, MR angiography of inflow and outflow vessels of the lower extremities is still a challenge since the total length of the peripheral vasculature (1000-1200-mm) exceeds the maximum length obtainable with one field of view. In order to cover the entire peripheral vasculature, multiple stations have to be imaged in one exam. This can be contradictory to the basic principles for successful GdE MR angiography: imaging during bolus passage to ensure high intra-arterial concentration of contrast material, imaging during breathhold in areas where breathing can cause motion artifacts, and imaging before venous enhancement (due to recirculation) and surrounding tissue enhancement (due to tissue dispersion of contrast material) occurs. Concerning the latter basic rule, a second or even a third injection of a bolus of contrast material can degrade image quality, as both veins and surrounding tissue already contain contrast material from (a) previous injection(s) and will be more difficult to saturate. Therefore, imaging multiple stations cannot be simplified to the use of multiple injections (one injection for each station imaged). To overcome this drawback, different research groups came up with different ideas. A useful classification for the different techniques is the strategy used to image the passing bolus. There are three main strategies: bolus catch technique, bolus chase technique and bolus track technique.

The purpose of this article is to explain working mechanisms of the three bolus imaging strategies for imaging both inflow and outflow vessels of the lower extremities, to show their advantages and disadvantages, and to review results described in literature in imaging patients using these techniques.

\section{Timing the Bolus}

For each of the three bolus imaging strategies, timing arrival of the bolus of contrast material in the imaged volume is essential in order to minimize the volume of the bolus, to minimize the chance of venous enhancement, and to ensure an even and high concentration of contrast material in the imaged volume during the sampling of the center of K-space (contrast profiles) [8]. In order to synchronize start of the acquisition with the arrival of the bolus of contrast material in the volume of interest, several techniques are available. The most simple technique is a timing sequence. This is a dynamic T1-weighted sequence with which one single slice is acquired every 1-2 seconds, consecutively. To determine arrival-time for the pelvic arteries, this slice can be planned proximal to the aortic abdominal bifurcation. The use of an axially planned slice is probably easiest and fastest, since no careful positioning in 
anteroposterior or left-right direction is needed. If an axially planned slice is used, a presaturation slab cranial to the imaged slice should be applied to prevent inflow effects from arterial blood. A test bolus of 2-mL of contrast material (Gd-DTPA with a concentration of $0.5-\mathrm{mmol} / \mathrm{mL}$ ) with a subsequent flush of $30-\mathrm{mL}$ of $0.9 \%$ saline (to flush contrast material from tubing and veins), is injected with the same injection rate as planned for the GdE MR angiographic scan. The dynamic scan and the injection of the test bolus (and subsequent normal saline flush) are started simultaneously. The scanned slices are then evaluated for an increase in signal intensity of the abdominal aorta, representing the arrival of the test bolus. The number of the slice in which the elevated intra-aortal signal intensity is seen, multiplied by the temporal resolution per slice is the arrival-time of the bolus of contrast material in the planned volume. In order to determine the scan-delay time, which is defined as the time passed between start of intravenous injection and start of the contrast-enhanced scan, it is important to keep in mind the prescribed K-space profile acquisition order for the threedimensional GdE MR angiographic scan. As this depends on both $\mathrm{K}$-space acquisition strategy (low-high/centric ordered or linear K-space filling) and the scanner hard- and software, a definite answer is to be obtained from the hardware manufacturers. The arrival of contrast material is determined prior to the actual GdE $\mathrm{MR}$ angiographic sequence, therefore this technique can be somewhat imprecise due to intrapatient variation of circulation. To determine the arrival-time below the aorta, a carefully planned coronal slice, encompassing all arteries of interest is best used, as smaller vessel diameter in the runoff arteries and possible vascular pathology, make detecting the arteries in axially oriented slices very cumbersome. Other more automated techniques have been proposed and are commercially available [2-4]. Basically relying on the same mechanism as the simple timing sequence, the purpose of these newer techniques is to start the acquisition of the GdE MR angiographic sequence when the signal intensities in the arteries of interest have reached a certain level, either fully automated [2], or operator dependent [4].

\section{Bolus Catch}

The first multiple-station technique discussed can be classified as a bolus catch technique. As mentioned above, timing the bolus arrival in the lower extremities can be very cumbersome due to the small diameter of the vessels of interest and becomes even more cumbersome in case of severe pathology. To overcome this problem several research groups have attempted to catch the passing bolus by consecutive acquisition of the same GdE MR angiographic scan for each field of view. By acquiring the same volume consecutively, there will always be a volume containing mainly arterial information. As yet three groups documented techniques which can be classified as bolus catch strategies. All of them used multiple injections with subsequent imaging of consecutive GdE MR angiographic scans and the use of precontrast scans for subtraction (fig. 1). Rofsky et al acquired one precontrast (mask) scan and only two post contrast scans [5]. They acquired two stations with two separate injections of contrast material. In 15 patients they found sensitivity and specificity figures for detecting hemodynamically significant stenoses of $97 \%$ and $96 \%$, respectively. They did not image the runoff arteries down to the feet. 


\section{Consecutively acquired volumes, used to catch the passing bolus of contrast material}

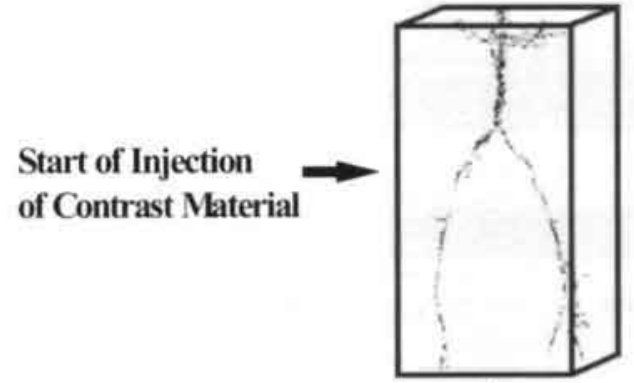

A

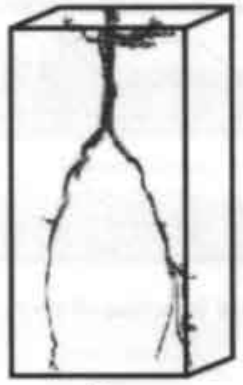

B

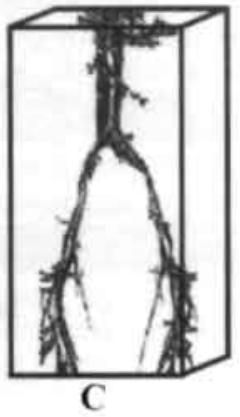

Figure 1. Schematic illustration shows the principle of bolus catch MR angiography for multiple station MR angiography without the problem of mistiming the bolus. After injection of contrast material the consecutively acquired volumes are evaluated for the presence of solely arterial information. A: first volume acquired too early, therefore the arterial enhancement is too low. B: maximum concentration of the bolus of contrast material is caught in the arterial phase. C: volume acquired too late, showing both arterial and venous enhancement.

Watanabe et al, followed a similar protocol but after the precontrast (mask) volume, they acquired five post-contrast volumes, covering the entire peripheral vasculature [6]. They examined 12 patients and concluded that "the atherosclerotic lesions were clearly revealed on dynamic subtraction MR angiography and were subsequently confirmed by conventional radiographic angiography". Korosec et al also acquired useful arterial hemodynamic information with their time-resolved imaging of contrast kinetics (TRICKS) technique [7]. With this technique after the precontrast volume, many 3D consecutive contrast volumes are acquired with ultrahigh temporal resolution $(\approx 5$ seconds per station). Although this technique has the potential to image the peripheral inflow and outflow arteries, a prospective study evaluating its feasibility in patients with peripheral vascular disease has not been published yet.

The main pitfall with the bolus catch strategy has been mentioned above. After the first injection of contrast material, background tissue and veins will have increased signal intensities due to residual and recirculating contrast material and thus vesselto-background contrast in subsequent scans will diminish. This problem can be overcome by subtracting a precontrast (mask) volume from a contrast-enhanced volume. However, the subtraction technique cannot compensate for residual contrast material in arteries, resulting in lower vessel-to-background contrast when multiple injection of contrast materials are used.

\section{Bolus Chase}

The second multiple-station technique discussed can be classified as a bolus chase technique. With this technique the bolus of contrast material is chased down the legs using fast 2D thick slabs, imaging 3 to 4 stations. Again a subtraction technique was used to increase vessel-to-background contrast (fig. 2). 


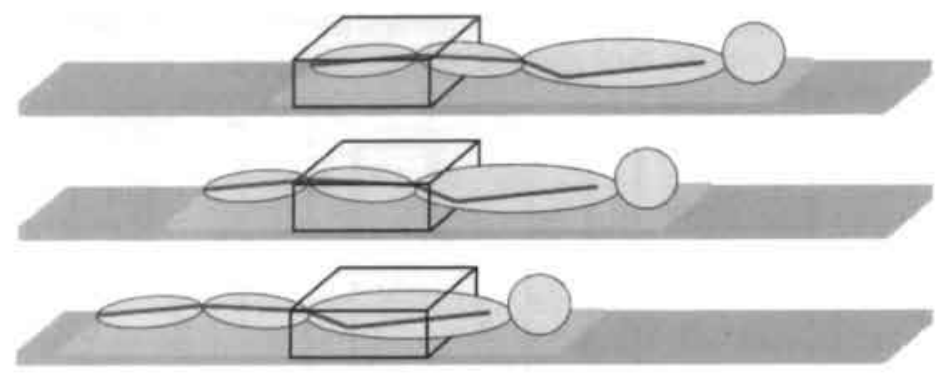

Start of injection of short bolus
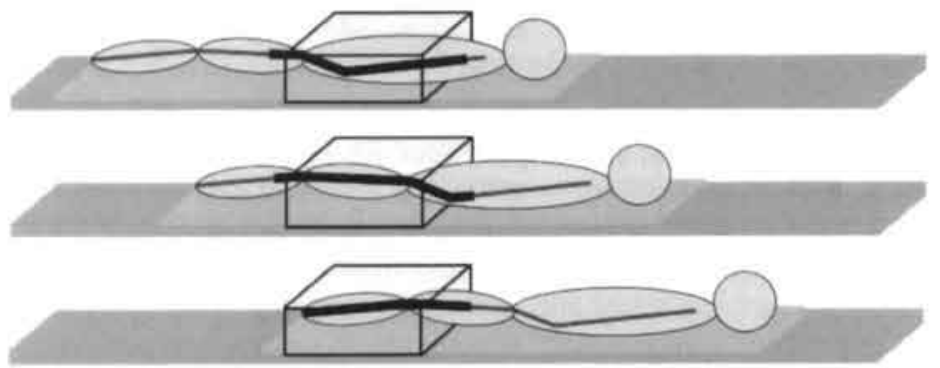

Figure 2. Schematic illustration shows the principle of bolus chase MR angiography for multiple station MR angiography. First three mask images are acquired, after which the injection of contrast material is started. Subsequently the short bolus that is chased down the legs. Fat black line: short bolus of contrast material caught in the imaged volume. Thin black line: arteries before or after bolus passage.

Wang et al pioneered this technique and examining 5 patients they concluded that "the peripheral arteries were well depicted in their entirety" [8]. Although the entire peripheral vascular tree is imaged, this technique has the disadvantage of acquiring only 2D data and problems can occur if arrival-time of contrast material varies per leg, risking mistiming of the bolus particularly in the lower legs. A similar bolus chase technique acquiring 3D volumes with dynamic $\mathrm{K}$-space filling as proposed by Lee et al improves resolution as compared to the 2D bolus chase technique [9]. Again, disadvantage of this technique is the chance of mistiming of the bolus.

\section{Bolus Track}

The third multiple-station technique discussed can be classified as a bolus track technique. This technique developed at our institution and independently from our group by Dr J.F.M. Meaney from Leeds General Infirmery Hospital, the bolus of contrast material is tracked down the legs, while moving the table rapidly in between volume acquisition. Therefore, we call this technique the Moving-Bed Infusiontracking technique or MoBI-track [10]. During the infusion of one long single bolus of contrast material, all three stations are imaged consecutively, covering the entire peripheral vasculature (fig. 3). The difference with the bolus chase technique is that due to the fact that one long single bolus of contrast material is used, differences in arrival-time per leg is not a problem. This technique is possible on a "conventional" MR scanner (gradient strength $10 \mathrm{mT} / \mathrm{m}$, rise time $1-\mathrm{msec}$ ). In 28 patients we found 
sensitivity and specificity figures for detecting hemodynamically significant stenoses of $93 \%$ and $98 \%$, respectively [10].

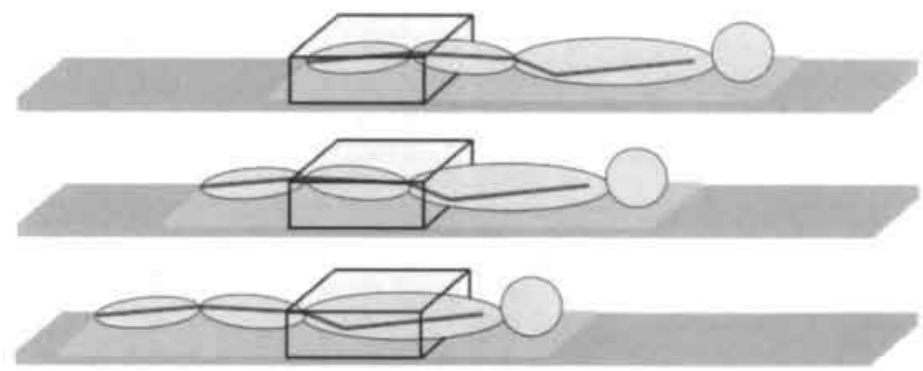

Figure 3. Schematic illustration shows the principle of bolus track MR angiography for multiple station MR angiography. First three mask volumes of the lower leg, upper leg and pelvic region are acquired. Than the infusion of contrast material is started. Note the long stretched slowly injected single bolus that prevents mistiming in any imaged station. Fat black line: long stretched bolus of contrast material caught in the imaged volume. Thin black line: arteries before or after bolus passage.

Disadvantage of this technique is the increased risk of venous enhancement, especially in the lower legs. Evaluating image interpretability in a retrospective study in 131 patients however, we found that poor vessel segment interpretability occurred in only $5 \%$ of all lower leg vessel segments (per leg 6 segments: both upper and lower part of the anterior tibial, posterior tibial and peroneal arteries).

\section{New and Future Developments}

Since the introduction of the above described techniques, new technical innovations have arisen. These innovations can be used to optimize the documented techniques, For bolus catch MR angiography using the more sophisticated timing techniques like Bolustrak $^{\$}$ and fluoroscopic triggering, the passing bolus of contrast material does not have to be caught anymore but can be detected real time. Even more so, the volume of the bolus per station probably can be diminished as accurate start of data acquisition and specific K-space sampling can take full advantage of a smaller bolus. As catching the bolus will no longer be relevant the bolus catch strategy probably should be renamed to multiple station with multiple injection peripheral GdE MR angiography. Another advantage of the new timing techniques can be the use of hemodynamic information from the acquired timing data. 
For the bolus-track strategy, we now have developed an optimized MoBI-track technique, taking full advantage of a fast gradient system (1.5 Tesla, gradient strength $21 \mathrm{mT} / \mathrm{m}$, risetime $0.3 \mathrm{sec}$ ) and optimized $\mathrm{K}$-space sampling. Therefore we are able to reduce the total acquisition time of the three acquired volumes (pelvic, upper leg and lower leg station) by $35 \%$ (down to 70 seconds) and simultaneously increasing spatial resolution, signal-to-noise (SNR) and contrast-to-noise ratios (CNR). Preliminary data shows that the increase in temporal resolution effectively eliminates venous enhancement, specifically in the lower leg region (fig. 4).

A

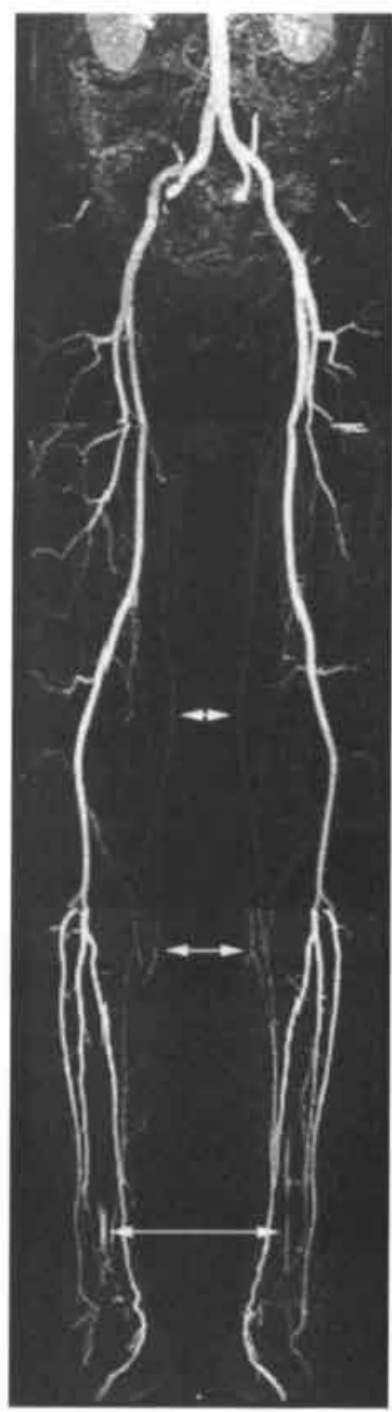

B

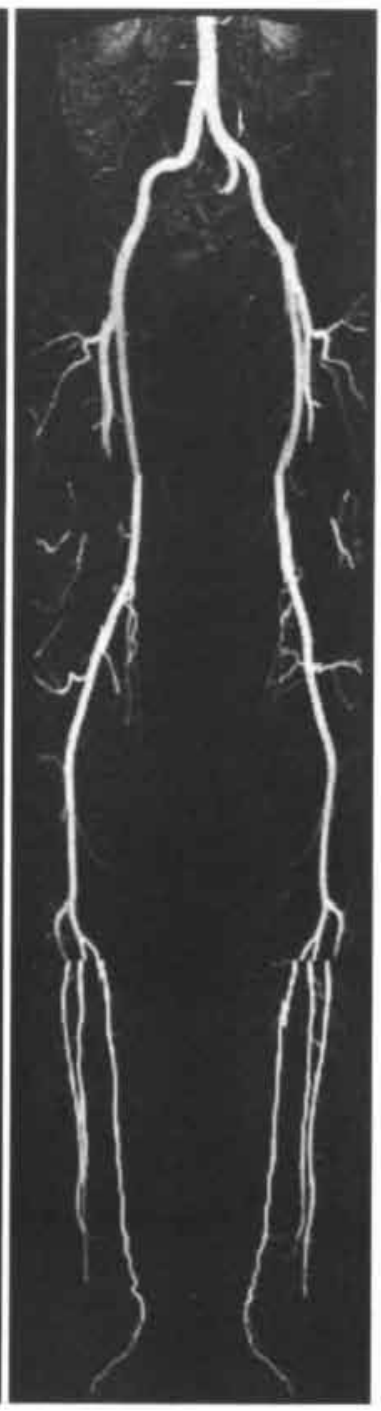

Figure 4. Maximum intensity projection images of a 38-year-old healthy volunteer. A: images obtained with the standard MoBItrack MR angiographic technique. B: images obtained with the optimized MoBI-track technique $(35 \%$ faster and increased resolution). Note the complete absence of any venous enhancement (arrows in image A) in image $B$.

Other innovations consist of the use of dedicated lower extremity coils to increase SNR and CNR. It is our experience that part of this increase in these ratios can also be used to increase spatial resolution, as spatial resolution in the peripheral arteries often is restricted by insufficient SNR and CNR (highest resolution is often not used, as SNR and CNR will drop below an acceptable value). Figure 5 demonstrates the increase in SNR and CNR using a dedicated lower extremity coil as compared to using the body coil for signal reception. Preliminary data of an ongoing study at our institution, comparing ultrahigh spatial resolution contrast-enhanced MR angiography, time-of-flight MR angiography and diagnostic $\mathrm{X}$-ray angiography (including digital subtraction angiography) of the lower leg 
arteries with intra-operative diagnostic X-ray angiography as the standard of reference, shows that GdE MR angiography is the preferred technique.

A

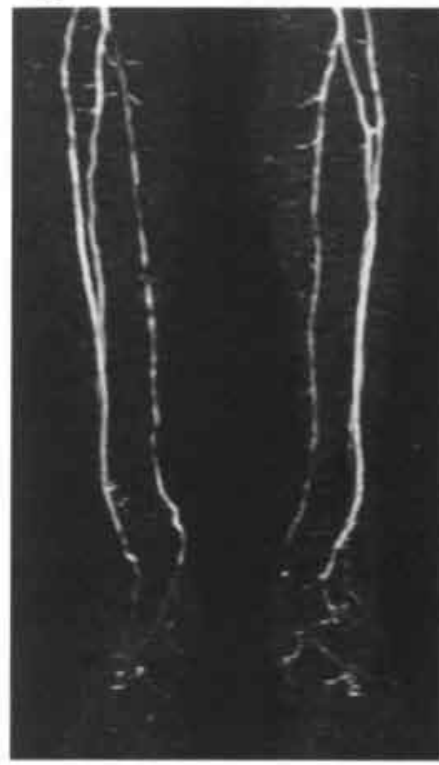

B

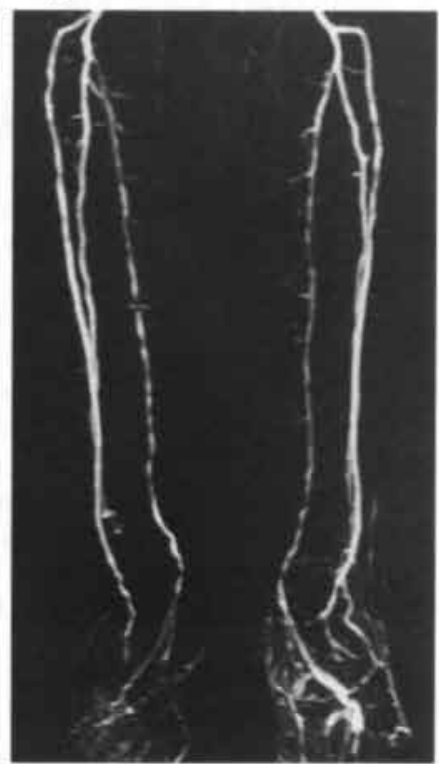

Figure 5, Maximum intensity projections of the lower leg region. A: scan acquired with the body coil for signal transmission and reception. B: scan acquired with the body coil for signal transmission and a dedicated lower extremity phased array coil for signal reception. Note the visual increase in vesselto-background contrast.

A final new development worth mentioning is the use of bloodpool agents for imaging the peripheral arteries. As bloodpool agents have a prolonged intravascular half-life, the acquisition times are not restricted to imaging during bolus passage. Therefore ultrahigh resolution images can be obtained. Disadvantage of these agents is the equal arterial and venous enhancement, making distinguishing arteries from veins cumbersome. This is particularly true for the lower leg arteries, as arteries and veins run in close proximity of each other. Figure 6 demonstrates the improved depiction of a stenosis when imaged with bloodpool-enhanced MR angiography as compared to GdE MR angiography.

A

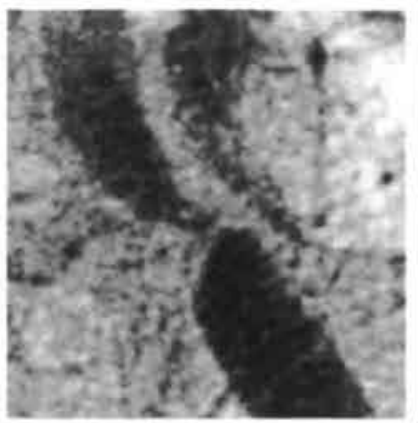

B

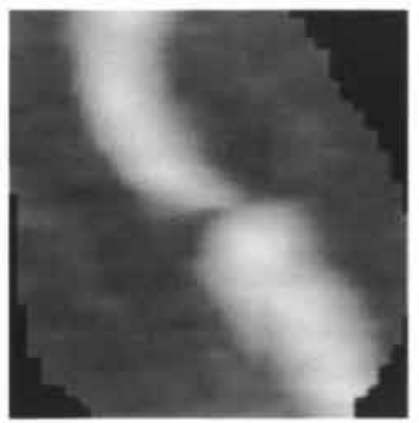

C

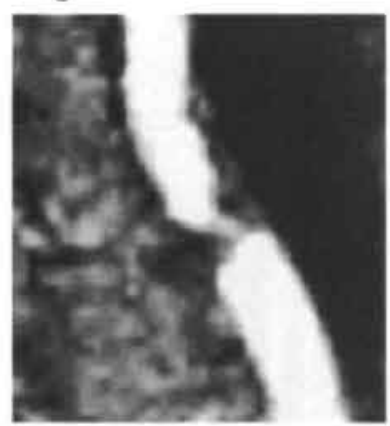

Figure 6. Images of a tight stenosis in the external iliac artery in a 64-year-old man. A: digital subtraction angiography. B: Gd-DTPA-enhanced MR angiography. C: bloodpool-enhanced (NC100150) MR angiography. Note the excellent depiction of the stenosis using bloodpool-enhanced MR angiography. 


\section{Conclusion}

In our institution Gd-DTPA-enhanced MR angiography of the peripheral arteries has ousted time-of-flight MR angiography and in many cases also ousted diagnostic Xray angiography. Several techniques can be used to image the peripheral arteries. As better hard- and software becomes available, these techniques will certainly be important tools in the diagnostic workup of patients with peripheral vascular disease. 


\section{References}

1. Maki JH, Prince MR, Londy FJ, Chenevert TL (1998) The effects of time varying intravascular signal intensity and $\mathrm{k}$-space acquisition order on three-dimensional MR angiography image quality. JMRI 6: 642-651.

2. Foo TKF, Saranathan M, Prince MR, Chenevert TL (1997) Automated detection of bolus arrival and initiation of data acquisition in fast, three-dimensional, gadoliniumenhanced, MR angiography. Radiology 203: 275-280.

3. Wilman AH, Riederer SL, King BF, Debbins JP, Rossman PJ, Ehman RL (1997) Fluoroscopically triggered contrast-enhanced three-dimensional MR angiography with elliptical centric view order: application to the renal arteries. Radiology 205: 137-146.

4. Kouwenhoven M, Groen J, de Graaf F, Meaney JFM, de Haan MW, Ho KYJAM, van Engelshoven JMA (1998) Bolustrak; timing of 3D contrast-enhanced MRA using single thick slice imaging with real time complex subtraction. Proceedings of the 15th annual meeting of the ESMRMB: 184.

5. Rofsky NM, Johnson G, Adelman MA, Rosen RJ, Krinsky GA, Weinreb JC (1997) Peripheral vascular disease evaluated with reduced-dose gadolinium-enhanced MR angiography. Radiology 205: 163-169.

6. Watanabe Y, Dohke M, Okumura A, Amoh Y, Ishimori T, Oda K, Dodo Y (1998) Dynamic subtraction MR angiography: first-pass imaging of the main arteries of the lower body. AJR Am J Roentgenol 170: 357-360.

7. Korosec FR, Frayne R, Grist TM, Mistretta CA (1996) Time-resolved contrastenhanced 3D MR angiography. Magn Reson Med 36: 345-351.

8. Wang Y, Lee HM, Khilnani NM, Trost DW, Jagust MB, Winchester PA, Bush HL, Sos TA, Sostman HD (1998) Bolus-chase MR digital subtraction angiography in the lower extremity. Radiology 207: 263-269.

9. Lee HM, Wang Y (1998) Dynamic k-space filling for bolus chase 3D MR digital subtraction angiography. Magn Reson Med 40: 99-104.

10. Ho KYJAM, Leiner T, de Haan MW, Kessels AGH, Kitslaar PJEHM, van Engelshoven JMA (1998) Peripheral vascular tree stenoses: evaluation with movingbed infusion-tracking MR angiography. Radiology 206: 683-692. 


\section{Chapter 9}

General Discussion 



\section{General Discussion}

Since its introduction by Wedeen et al, MR angiography has been a promising technique for imaging peripheral arteries. First and foremost, it was a noninvasive technique which held the promise to obtain both hemodynamic and morphologic information about the vascular tree. This thesis dealt with the feasibility to obtain adequate morphologic information of the peripheral vascular tree. However, the former (conventional) MR angiographic techniques, of which time-of-flight was most widely used, were only able to detect and grade peripheral vascular obstructive disease suboptimally. This was mainly caused by the virtue of which time-of-flight MR angiography exists. Flow being the cause of the arterial signal, it also is the instigator of artifacts degrading image quality and interpretability. Although not examined in this thesis, phase contrast MR angiography suffers from the same artifacts as time-of-flight MR angiography.

At the time the first study of this thesis was performed, a new descendant of MR angiography arose. Contrast-enhanced MR angiography, depending on gadoliniumchelates for its vessel-to-background contrast, did not suffer from the flow dependent artifacts, as was the case with time-of-flight MR angiography. Although necessitating intravenous administration of a foreign substance, the safety profile of gadoliniumchelates is extremely good. Adverse event rates reported in a group of over 34.000 patients, were in the order of $0.003-0.01 \%$. In many centers, contrast-enhanced MR angiography has changed the diagnostic workup for several vascular areas (thoracie and abdominal aorta, pulmonary and renal arteries), as it ousted the other modalities like Duplex ultrasonography and diagnostic X-ray angiography.

Although the results for imaging the pelvic arteries were very promising, contrastenhanced MR angiography was restricted to the acquisition of only one single field of view, covering $400-500-\mathrm{mm}$ of the peripheral vascular tree. This would comprise only $1 / 3$ of the entire peripheral vascular tree. In order to image both inflow and outflow vessels of the lower extremities, multiple field of views would have to be acquired. Not only would this increase examination time threefold because for each field of view, the patient would have to be repositioned, and new scout views would have to be obtained in order to plan the next three-dimensional volume, the main limitation of multiple field of views is the necessity for multiple injections of contrast material. Besides increasing the total volume of (expensive) contrast material needed, it also could potentially decrease vessel-to-background contrast in subsequent acquisitions due to residual contrast material in both veins and surrounding tissues. Although subtraction of a pre-injection mask volume could compensate for part of these effects, residual contrast material in arteries themselves would still result in suboptimal vessel-to-background contrast.

To overcome these restrictions of imaging only one single field of view, while at the same time still using only one bolus of contrast material, we developed a new MR angiographic technique called Moving-Bed Infusion-tracking MR angiography (MoBI-track MR angiography). With this technique, three separate field of views can be acquired during the slow infusion of one single bolus of contrast material (30-40$\mathrm{mL}$ ). With the use of precontrast mask volumes for subtraction, vessel-to-background contrast is increased, to compensate for the relatively low intra-arterial concentration of contrast material due to the low injection rate of $0.3-\mathrm{mL} / \mathrm{sec}$. First results in both 
volunteers and patients showed good delineation of the entire peripheral vascular tree. Comparing MoBI-track data of patients with X-ray angiography, showed excellent agreement for detecting and grading peripheral vascular obstructive disease of the entire inflow and outflow trajectory. Evaluation of image interpretability in a large patient population revealed good image quality in a vast majority of patients and vessel segments (poor vessel segment interpretability occurred in only $2.5 \%$ of all evaluated vessel segments). As poorly interpretable vessel segments mainly occurred in the lower legs, caused by venous enhancement, preventing this is our next major goal.

Beside our MoBI-track technique, others have developed different approaches for imaging the entire peripheral vascular tree. If MoBI-track MR angiography can be considered as a bolus-track technique (keeping track of the bolus of contrast material as it travels down the legs), a faster approach is the bolus-chase technique, that chases a fast injected highly concentrated bolus of contrast material down the legs. However, main disadvantage of this technique is mistiming of the bolus of contrast material, particularly in the lower legs, caused by the differences in blood flow per leg. A third technique currently employed can be identified as a bolus-catch techniques. By acquiring multiple consecutive volumes, the passing bolus of contrast material is caught in the image volume. Although this technique can be optimized by using the newly developed real time bolus detection systems, its main disadvantage of multiple injections of contrast materials, necessary to cover the entire peripheral vasculature, still limits its ability to image the entire peripheral vascular tree.

As both Bolus-chase and bolus-catch techniques described above require state-ofthe-art MR equipment, MoBI-track MR angiography can be performed on a conventional MR systems (without ultra fast gradients). Even more so, MoBI-track MR angiography has been adopted by our scanner manufacturer (Philips Medical Systems, Best, The Netherlands) who improved its convenience and implemented new hard- and soft-ware possibilities. Therefore, this technique can now be performed on any (Philips) MR scanner, equipped with "Mobitrak" (That is MoBItrack without the "c" in track; Philips' name for the developed software package) software. Multiple centers are now testing the MoBI-track technique (over 10 different hospitals) using the "Mobitrak" software, and first results from these centers corroborate our results. As other hardware manufacturers (for instance General Electric Medical Systems) have copied the idea of MoBI-track MR angiography, it is to be expected that within a year many more centers will be using this technique.

\section{Future directions}

As mentioned in the section "New and Future developments" of chapter 8, new improvements of the MoBI-track technique have enabled us to reduce acquisition time remarkably. The original MoBI-track technique took 2 minutes to acquire all three stations (pelvic, upper leg and lower leg stations). However, with the optimized technique, these three stations can be acquired in little over 1 minute with increased resolution for all stations as well as improved signal and contrast-to-noise ratios. Therefore, the most prominent factor disturbing image quality in MoBI-track MR angiography, being venous enhancement in the lower legs, is likely to be eliminated. 
Even more so, as improvements on hard- and soft-ware are evolving rapidly, the MoBI-track technique will also become simpler and less operator dependent.

This thesis mainly dealt with imaging the entire peripheral vascular tree. However, more "station-dedicated" techniques, can significantly improve evaluation accuracy and image quality. The combination of newly developed techniques like automated bolus detection, fast gradient systems, dedicated extremity coils and a specialized acquisition technique (specific K-space sampling strategy [low-high]), allowed us to acquire ultrahigh resolution gadolinium-enhanced MR angiographic threedimensional volumes with voxel volumes of $1-\mathrm{mm}^{3}$, with superior signal-to-noise and contrast-to-noise ratios. Figure 1 demonstrates the lower legs of a healthy volunteer where even the smallest arteries are clearly delineated (even in the feet).

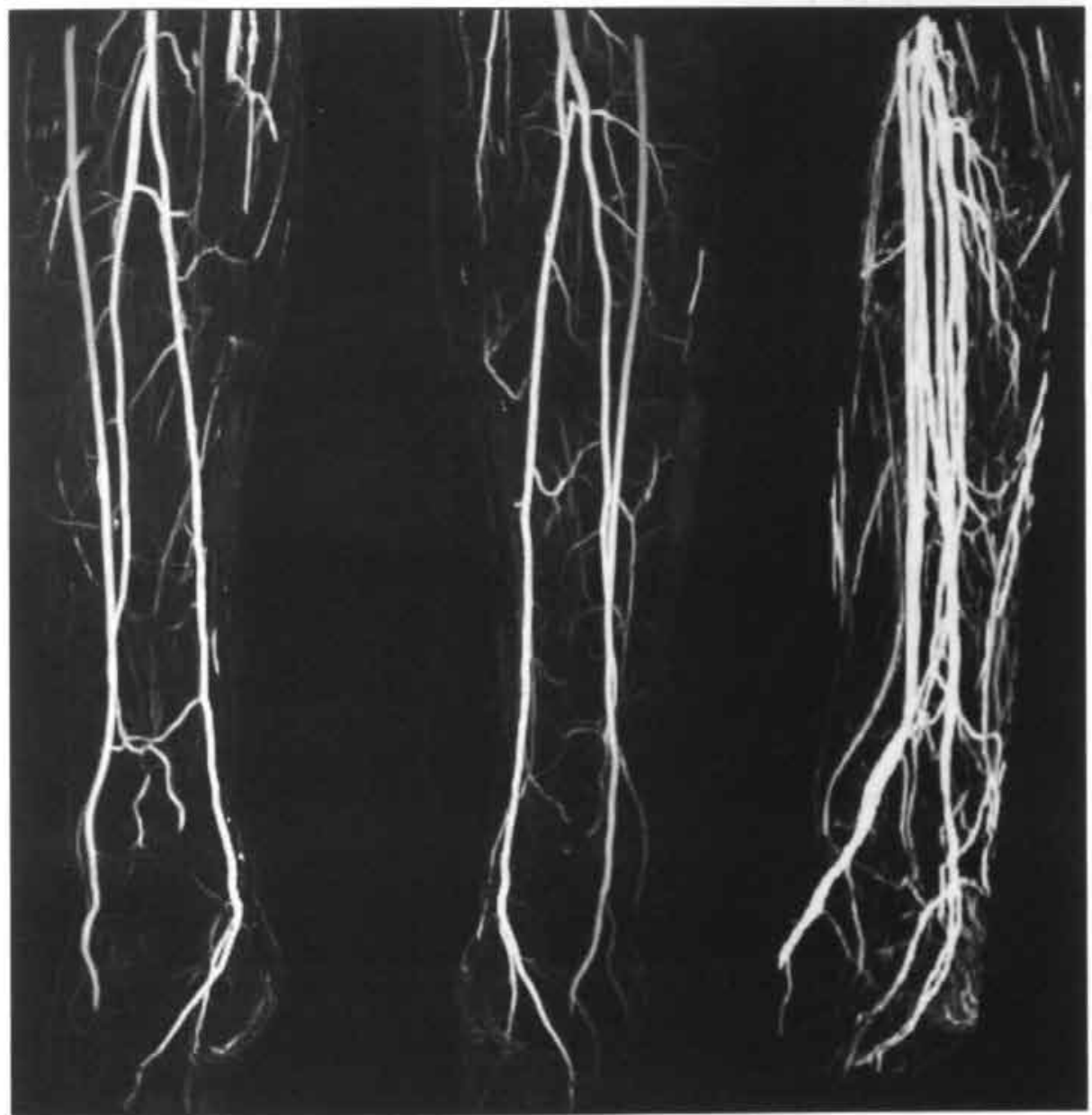

Figure 1. Ultrahigh resolution gadolinium-enhanced MR angiography of the lower legs in a healthy volunteer. Note the excellent delineation of even the smallest arteries because a voxel volume of $1-\mathrm{mm}$ was used. \{Three-dimensional views of the lower leg arteries can be seen on the Image-CD\}. 
Other ultrahigh resolution acquisition strategies will probably arise within the next few years as bloodpool agents will become commercially available.

A final note has to be made on postprocessing the acquired data. As yet all our studies consisted of the evaluation of maximum intensity projection (MIP) images and sometimes original partitions (slices). However, as contrast-enhanced MR angiography is capable of acquiring three-dimensional data, dedicated software packages, exploiting the three-dimensional nature of the acquired data is likely to improve interpretation accuracy and interpretability. First steps towards dedicated software packages are being made and first results look promising.

\section{Clinical Impact}

The MoBI-track technique changed the scope of peripheral MR angiography as it enabled imaging three different stations using only one single bolus of contrast material on a conventional MR system. Therefore a peripheral outflow study encompassing the entire peripheral vascular tree can be performed within several minutes on an outpatient basis without the necessity for patient pre-preparation or after care. Even more so, despite the use of an intravascular contrast agent, the procedure has such a high safety profile, that screening of patients for peripheral vascular obstructive disease becomes feasible. This might create interesting perspectives in the evaluation of progressive disease, evaluation of both interventional and non-interventional theranv, as well as broad enideminlosical studies. However, the clinical impact MoBI-track MR angiography can have on patients with peripheral vascular obstructive disease, depends on its ability to compete with both diagnostic X-ray angiography as well as Duplex ultrasonography. Although the inplane spatial resolution of MoBI-track MR angiography is lower as compared to X-ray angiography, its three-dimensional nature, allows reconstructions of views in any desired obliquity. Therefore, it is likely that peripheral vascular obstructive disease is more accurately depicted using MoBI-track MR angiography (fig. 7, chapter 5). Even more so, as the MoBI-track technique can rely on imaging the arteries as long as venous or surrounding tissue enhancement does not occur, it is not restricted to the very short bolus of contrast material as used with X-ray angiography. This means that slow filling arteries as seen with retrograde filling beyond a complete obstruction or more distally located (lower leg) arteries, can be depicted without the chance of mistiming the bolus (imaging to early, fig. 9, chapter 5).

The competition with Duplex ultrasonography seems in favor of the afore mentioned as this technique is cheaper as a conventional MR examination. However, the total patient handling time, using the MoBI-track technique is only 15 to 20 minutes, and the costs for MRI are mainly dictated by the maintenance costs for the MR scanner. Therefore reducing scantime can effectively reduce the costs per examination. Combined with the higher sensitivity for detecting and grading stenoses in the entire peripheral vascular tree, the ability to acquire morphological data, as well as its lesser operator dependancy, MoBI-track MR angiography might proof a serious competitor of Duplex ultrasonography. A long term randomized trial, comparing the two techniques as part of the diagnostic workup of a patient with complaints of 
peripheral vascular obstructive disease, might provide a more objective view on this matter.

\section{Conclusion}

Peripheral MR angiography is a good alternative for depiction of pathology in patients with complaints of peripheral vascular obstructive disease. Using the MoBItrack technique, peripheral MR angiography might even be able to oust diagnostic $\mathrm{X}$ ray angiography and Duplex ultrasonography. 
Chapter 9 
Summary 



\section{Summary}

Atherosclerotic disease of the lower extremities is a common disorder in western society. Its debilitating nature calls for accurate diagnosis and treatment. The gold standard for diagnosing this disease, by depiction of vessel morphology, is diagnostic $\mathrm{X}$-ray angiography (either conventional or digital subtraction angiography). However, the invasive nature of this technique and the possible harmful effects of iodinated contrast agents have led to the idea that noninvasive MR angiography might be a good alternative for acquiring information about vessel morphology. Most extensively studied was time-of-flight MR angiography. Although first results with this technique were encouraging, it is now apparent that time-of-flight MR angiography is hampered by its flow dependency which is also the major instigator of artifacts. With the introduction of minimally invasive contrast-enhanced MR angiography, using gadolinium-chelates to reduce the $\mathrm{T} 1$ of blood, image quality has improved tremendously. Even more so, using contrast-enhanced MR angiography, high resolution three-dimensional data of the entire peripheral vascular tree can be obtained within several minutes, which might make MR angiography a true competitor of X-ray angiography as a diagnostic tool in the clinical workup of a patient with complaints of peripheral vascular obstructive disease.

In chapter 2, the working mechanisms and usefulness of both time-of-flight and contrast-enhanced MR angiography are explained.

In chapter 3, two inflow MR angiographic pulse sequences (turbo "field-echo" with inversion prepulse and fast "field-echo" without inversion prepulse) obtained both with and without systolic synchronization were compared. All pulse sequences were also compared with X-ray angiography as the standard of reference. In total thirtyone consecutive patients who were scheduled for X-ray angiography because of symptomatic atherosclerotic obstructive disease of the iliac and/or femoral arteries underwent MR angiography using the four different MR angiographic techniques. Systolic synchronization proved to be essential for adequate image quality and although there was no statistically significant difference in detecting and grading hemodynamically significant stenoses between systolic synchronized fast "fieldecho" and turbo "field-echo" techniques, the latter produced the best image quality both objectively and subjectively. Therefore we concluded that the systolicsynchronized turbo field-echo pulse sequence is superior to the other three MR angiographic techniques.

In chapter 4 , the best time-of-flight technique as determined in chapter 3 (the systolic-synchronized turbo "field-echo" technique) was compared with both subtracted and nonsubtracted gadolinium-enhanced MR angiography using X-ray angiography as the standard of reference. In total twenty-eight patients with intermittent claudication were included in this study. Sensitivity and specificity for grading of hemodynamically significant stenoses ( $250 \%$ lumen reduction) on subtracted MR angiograms were $94 \%$ and $93 \%$, respectively. Sensitivity as determined on subtracted images was significantly higher as compared to that determined on time-of-flight images $(\mathrm{p}<.05)$ but not to that as determined on nonsubtracted images. Contrast-to-noise ratios of subtracted images was significantly higher compared to that of nonsubtracted images $(\mathrm{p}<.05)$ but not to that of time-offlight images. There was good correlation between stenosis length measurements on 
gadolinium-enhanced MR angiograms and those on conventional angiograms. Therefore our conclusion was that subtracted MR angiography is superior to systolicsynchronized time-of-flight MR angiography for imaging of iliac and upper femoral arteries and provides higher contrast-to-noise ratios, fewer artifacts, and easier image interpretability than nonsubtracted MR angiography.

As gadolinium-enhanced MR angiography was restricted to imaging just one single field of view, depicting only $1 / 3$ of the entire peripheral vascular tree, we developed a new technique as described in chapter 5, called the Moving Bed Infusion tracking (MoBI-track) technique, enabling us to image the entire peripheral vasculature in one session. To evaluate the feasibility of this technique, 15 healthy volunteers and 28 patients with intermittent claudication underwent MR angiography using the MoBItrack technique. The MoBI-track technique consists of acquiring three pre-contrast mask volumes of the lower leg, upper-leg and pelvic regions with subsequent acquisition of contrast volumes during the infusion of one single bolus of gadolinium-chelates of the pelvic, upper leg and lower leg region with fast intervolume table movement. Subsequent subtraction of mask volumes from gadolinium-enhanced volumes yielded volumes with preferentially arterial information. Maximum intensity projection images were generated and image quality was evaluated subjectively and objectively. Maximum intensity projection images of the patients were compared with X-ray angiograms, which served as the standard of reference. Moving-Bed Infusion-tracking MR angiography proved to be a robust technique, and image quality on maximum intensity projection images was comparable with that on X-ray angiograms. Sensitivity and specificity for grading hemodynamically significant stenoses were $93 \%$ and $98 \%$, respectively, with excellent interobserver agreement. Therefore we concluded that Moving-Bed Infusion-tracking MR angiography can be used to image all peripheral arteries in just 4 minutes by using a small amount of contrast material on a conventional $1.5-\mathrm{T}$ MR imager.

In chapter 6, the image interpretability of MoBI-track MR angiographic data was evaluated retrospectively in 131 patients by determination of the prevalence of poor or suboptimal image interpretability, as well as determination of technique and patient related factors which influenced image interpretability. The 131 patients were divided into two consecutively examined groups because in between patient recruitement, we had an update of our MR scanner. To determine image interpretability a three-point subjective interpretability score (good, suboptimal or poor interpretability) was used to evaluate all vessel segments of the peripheral vascular tree (29 per patient). Using a multivariant logistic regression analysis, the influence of different technique and patient related factors was examined (inclusion period, inflow pathology, Fontaine classification, height of the lower legs, age, lower leg pathology, sex, and venous enhancement in the lower legs). The results showed poor image interpretability in only $2.5 \%$ of all evaluated vessel segments. Poor image interpretability occurred mainly in the lower legs $(5 \%$ of all lower leg vessel segments). In the pelvic and upper leg vessel segments hardly any poor vessel interpretability was found $(0 \%$ and $1 \%$, respectively). Main disturbing factor for image interpretation in the lower legs was venous enhancement. No other statistically significant factors were identified. Therefore we concluded that MoBI-track MR angiography is capable of producing good image interpretability in a majority of 
patients and vessel segments. If venous enhancement in the lower legs can be prevented by using more dedicated hard- and software equipment, image interpretability can become near perfect.

In chapter 7, we evaluated the feasibility of bloodpool-enhanced peripheral MR angiography using iron-oxide particles (NC100150, Nycomed, Norway) in order to obtain both first pass as well as ultrahigh resolution data of the peripheral vascular tree. The ultrahigh resolution bloodpool-enhanced MR angiographic data was compared with gadolinium-enhanced MoBl-track MR angiography, using $\mathrm{X}$-ray angiography as the standard of reference. In total 10 patients were included. Through subjective and objective methods (vessel visibility score, shaded surface rendering and SNR/CNR calculations) image interpretability of the pelvic and upper leg arteries prooved to be good. This finding was reflected in the excellent sensitivity and specificity figures of $95 \%$ and $95 \%$, respectively, for detecting and grading hemodynamically significant stenoses on the bloodpool data (100\% en $98 \%$, respectively for gadolinium-enhanced MR angiography). In the lower legs, arterial and venous separation prooved to be cumbersome, which led to a poor interpretability score for this region. By using first-pass data of bloodpool-enhanced MR angiography, which was also acquired, it was possible to adequately separate arteries from veins in some patients.

In conclusion we stated that the depiction of peripheral vascular pathology using ultrahigh resolution bloodpool-enhanced MR angiographic data is feasible for both the pelvic and upper leg region, but results in the lower leg region are suboptimal.

Finally in chapter 8 , the current developments in peripheral MR angiography are highlighted. The main three strategies employed to image the entire peripheral vasculature can be classified as either bolus catch, bolus chase or bolus track techniques. The bolus catch technique aims at catching the passing bolus of contrast material by consecutive acquisition of volumes with high temporal resolution. The bolus chase technique, chases the short and highly concentrated bolus of contrast material down the legs. Finally the bolus track technique (MoBI-track MR angiography) keeps track of the long and lesser concentrated bolus of contrast material as it passes down the legs. Of these three techniques, the Bolus track technique is most widely used and is currently being copied by other hardware manufacturers. 
Samenvatting 



\section{Samenvatting}

Atherosclerosis van de onderste extremiteiten is een veel voorkomende ziekte in de westerse wereld. De ondermijnende aard van deze ziekte vraagt om een accurate diagnose en therapie. De gouden standaard voor het diagnosticeren van deze ziekte door middel van het afbeelden van de vaat-morfologie is diagnostische röntgen angiografie (zowel conventionele als digitale subtractie angiografie). Edoch, de invasieve aard van deze techniek en de mogelijke schadelijke effecten van joodhoudende contrastmiddelen, hebben tot het idee geleid, dat de noninvasieve MR angiografie een goed alternatief zou kunnen zijn voor het verkrijgen van informatie over de vaat-morfologie. Meest uitgebreid werd de instroom MR angiografie onderzocht ("time-of-flight" of "inflow" MR angiografie). Alhoewel de eerste resultaten van deze techniek zeer bemoedigend waren, is het nu duidelijk, dat instroom MR angiografie wordt gehinderd door zijn bloedstroom afhankelijkheid, dat niet alleen zorg draagt voor het verkregen arteriële signaal, maar tevens de veroorzaker is van storende artefacten.

Met de introductie van de minimaal invasieve contrast-versterkte MR angiografie, gebruik makend van gadolinium-chelaten, die de Tl van bloed drastisch kunnen verlagen is de beeldkwaliteit enorm verbeterd. Sterker nog, door gebruik te maken van contrast-versterkte MR angiografie is het thans mogelijk om hoge resolutie driedimensionale data te verkrijgen van de gehele perifere vaatboom in slechts enkele minuten. Hierdoor kan MR angiografie een volwaardig alternatief vormen voor röntgen angiografie als een diagnostische modaliteit in de klinische evaluatie van patiënten met klachten van perifere vasculaire obstructieve ziekten.

In hoofdstuk 2 werd het werkingsmechanisme en het nut van zowel instroom alsmede contrast-versterkte MR angiografie uitgelegd.

In hoofdstuk 3 werden twee instroom MR angiografische puls-sequenties, te weten de turbo "field-echo" met inversie prepulse en de fast "field-echo" zonder inversie prepulse, met elkaar vergeleken. Beide sequenties werden gebruikt met en zonder hartritme sychronisatie. Alle puls-sequenties werden vergeleken met röntgen angiografie als de gouden standaard. In totaal werden 31 achtereenvolgende patiënten onderzocht. Alle patiënten hadden klachten van claudicatio intermittens en met ultrageluids onderzoek bewezen vernauwingen van de bekken of bovenste beenvaten. Systolische synchronisatie bleek een onontbeerlijke toevoeging te zijn voor het verkrijgen van adequate beeldkwaliteit. Diagnostisch bleek er geen verschil te bestaan tussen de twee systolisch gesynchroniseerde technieken. De subjectieve alsook de objectieve beeldkwaliteit van de turbo "field-echo" sequentie bleek echter superieur te zijn aan die van de fast "field-echo" techniek. Derhalve concludeerden wij dat de systolisch gesynchroniseerde turbo "field-echo" sequentie superieur is aan de drie andere MR angiografische sequenties.

In hoofdstuk 4 werd de beste instroom sequentie, als bepaald in hoofdstuk 3 (systolisch gesynchroniseerde turbo "field-echo" seqeuntie) vergeleken met contrastversterkte MR angiografie, zowel met als zonder subtractie. Hierbij werd röntgen angiografie als gouden standaard gebruikt. In totaal werden 28 achtereenvolgende patiënten onderzocht. Alle patiënten hadden klachten van claudicatio intermittens en met ultrageluids onderzoek bewezen vernauwingen van de bekken of bovenste beenvaten. Sensitiviteit en specificiteit voor het graderen van hemodynamische 
significante stenosen op gesubtraheerde MR angiogrammen waren respectievelijk $94 \%$ en $93 \%$. De op de gesubtraheerde beelden bepaalde sensitiviteit was statistisch significant hoger in vergelijking met die bepaald op de instroom MR angiogrammen $(\mathrm{p}<.05)$, maar gelijk aan die bepaald op de niet gesubtraheerde contrast-versterkte MR angiogrammen. De contrast-ruis verhoudingen als bepaald op de gesubtraheerde contrast-versterkte MR angiogrammen was statisch significant hoger als die bepaald op de niet gesubtraheerde MR angiogrammen $(\mathrm{p}<.05)$ maar niet statistisch significant verschillend van die van de instroom MR angiogrammen. $\mathrm{Er}$ bleek een goede correlatie te bestaan tussen de lengte metingen van stenosen op contrast-versterkte MR angiogrammen en röntgen angiogrammen. Derhalve concludeerden wij dat gesubtraheerde contrast-versterkte MR angiografie superieur is aan systolisch gesynchroniseerde instroom MR angiografie voor het afbeelden van de bekken- en bovenste beenvaten en dat deze techniek betere contrast-ruis verhoudingen, minder artefacten en eenvoudiger interpretatie van de verkregen beelden biedt in vergelijking met de niet gesubtraheerde techniek.

Omdat contrast-versterkte MR angiografie beperkt was tot het afbeelden van slechts een niveau, waarbij niet meer dan $40-50 \mathrm{~cm}$ van de perifere vaatboom afgebeeld kon worden, hebben wij getracht een nieuwe techniek te ontwikkelen waarbij wel de hele vaatboom gevisualiseerd wordt. Deze techniek welke in hoofdstuk 5 beschreven staat noemen wij de Moving Bed Infusion tracking oftewel MoBI-track techniek. Om de haalbaarheid van deze techniek te onderzoeken, hebben wij 15 gezonde vrijwilligers en 28 patiënten met klachten van claudicatio intermittens onderzocht. De MoBI-track techniek bestaat uit de acquisitie van drie pre-contrast (masker) scans van de onderbeens-, bovenbeens- en- bekken-vaten, met daarop volgend de acquisitie van contrast-versterkte scans gedurende de infusie van één lang-gerekte bolus contrastmiddel (gadolinium-chelaten). De hierna volgende subtractie (masker scans van corresponderende contrast-versterkte scans) resulteert in scans welke preferentieel arteriële informatie bevatten. Maximum intensiteits projecties werden vervolgens zowel subjectief als objectief geëvalueerd. De maximum intensiteits projecties van de patiënten werden tevens vergeleken met röntgen angiografie. Sensitiviteit en specificiteit voor het graderen van hemodynamisch significante stenosen waren respectievelijk $93 \%$ en $98 \%$, met uitstekende inter-beoordelaar overeenkomst. Derhalve concludeerden wij dat de MoBI-track techniek gebruikt kan worden voor het afbeelden van alle relevante perifere vaten in slechts 4 minuten, door gebruik te maken van een kleine hoeveelheid contrastmiddel $(40 \mathrm{~mL})$ op een conventionele 1.5-Tesla MR scanner.

In hoofdstuk 6 werd retrospectief de interpreteerbaarheid van de MoBI-track data van 131 patiënten geëvalueerd door het bepalen van het voorkomen van slechte of suboptimale beeldkwaliteit, alsmede de bepaling van techniek en patiënt gerelateerde factoren, welke van invloed zouden kunnen zijn op de uiteindelijke beeldkwaliteit. De 131 patiënten werden onderverdeeld in twee achtereenvolgende groepen vanwege een tussentijdse vervanging van onze MR scanner. Om de beoordeelbaarheid van de vaatsegmenten ( 29 per patiënt) te kwantificeren werd gebruikt gemaakt van een driepunts subjectieve interpretatie score (goede, matige en slechte beoordeelbaarheid). Door gebruik te maken van een multivariant logistisch regressie model, werd de invloed onderzocht van zowel techniek alsmede patiënt gerelateerde factoren (inclusie periode, instroom pathologie, Fontaine classificatie, hoogte van de 
onderbenen, onderbeens-pathologie, geslacht en veneuze aankleuring in het onderbeen). De resultaten lieten slechte beoordeelbaarheid zien in $2.5 \%$ van alle beoordeelde vaatsegmenten. Slechte beoordeelbaarheid kwam met name voor in de onderbenen ( $5 \%$ van alle onderbeensvaatsegmenten). De bekken- en bovenbeensvaatsegmenten lieten nauwelijks slechte beoordeelbaarheid zien (respectievelijk $0 \%$ en $1 \%$ ). De meest storende factor voor slechte beoordeelbaarheid in de onderbenen bleek veneuze aankleuring te zijn. Benevens deze positief gecorreleerde bevinding, werden er geen andere storende factoren geïdentificeerd. Derhalve concludeerden wij dat het mogelijk is om met MoBI-track MR angiografie goede beoordeelbaarheid te verkrijgen in de meerderheid van de onderzochte patiënten en vaatsegmenten. Indien veneuze aankleuring in de onderbenen voorkomen kan worden door gebruik te maken van meer gespecialiseerde hard- en soft-ware, zal het aantal goed interpreteerbare vaatsegmenten de $100 \%$ naderen.

In hoofdstuk 7 hebben wij de toepasbaarheid van bloodpool-versterkte perifere MR angiografie geëvalueerd door gebruik te maken van ijzer-oxide deeltjes (NC100150, Nycomed, Noorwegen). Hierbij werden zowel "first-pass" alsmede ultrahoge resolutie data van het perifere systeem vergaard. De ultrahoge resolutie data werden vergeleken met gadolinium-versterkte MoBI-track MR angiografie voor het detecteren van hemodynamisch significante stenosen, waarbij röntgen angiografie als gouden standaard diende. In totaal werden 10 patiënten onderzocht. Door middel van zowel subjectieve als objectieve methoden (vaat-zichtbaarheid, shaded surface display, SNR/CNR), bleek de beoordeelbaarheid van bekken- en bovenbeens-vaten van de ultrahoge resolutie bloodpool-versterkte MR angiografie goed te zijn. Dit kwam tot uitdrukking in de goede sensitiviteits en specificiteits getallen voor het aantonen van hemodynamisch significante stenosen van respectievelijk $95 \%$ en $95 \%$ (respectievelijk 100\% en $98 \%$ voor de gadolinium-versterkte MR angiografie). In de onderbenen bleek de arterioveneuze separatie vaak een probleem, waardoor de beoordeelbaardheid op dit niveau onvoldoende bleek. Door gebruik te maken van de first-pass bloodpool-versterkte MR angiografische data, bleek het in een aantal gevallen mogelijke om adequate arterioveneuze segmentatie te verkrijgen in de ultrahoge resolutie bloodpool-versterkte MR angiografische data. Concluderend stelden wij dat perifere vasculaire pathologie gedetecteerd kan worden door gebruik te maken van ultra-hoge resolutie bloodpool-versterkte MR angiografie voor zowel bekken- als bovenbeens-vaten, maar dat de resultaten in de onderbeensregio suboptimaal zijn.

Tot slot werden in hoofdstuk 8 de drie belangrijkste strategiën beschreven, welke gebruikt worden voor het afbeelden van de perifere vaatboom. Deze kunnen geclassificeerd worden aan de hand van de manier waarop de bolus contrastmiddel gevisualiseerd wordt. De bolus catch techniek tracht de passerende bolus contrastmiddel te vangen door het snel achtereenvolgend acquireren van volumina. De bolus chase techniek, jaagt achter de passerende kortdurende en hoog geconcentreerde bolus contrastmiddel in het perifere vaatstelsel aan. Tot slot de Bolus-track techniek (MoBI-track MR angiografie) vervolgt de langgerekte en minder geconcentreerde bolus contrastmiddel wanneer deze door het perifere vaatstelsel stroomt. Van deze drie technieken is de Bolus track techniek het meest wijdt verspreid en gebruikt en wordt momenteel door andere hardware fabrikanten gekopieerd. 
Curriculum Vitae 



\section{Curriculum Vitae}

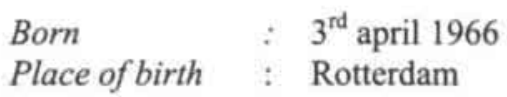

Education:

- 1978-1985 : High school: De Breul, Zeist / Herman Jordan Lyceum, Zeist / St. Adelbert college, Wassenaar / Bonaventura college, Leiden.

- 1985-1991 : Medicine, Free University of Amsterdam.

-1991-1994 : Internships, Free university of Amsterdam.

-1994-1995 : House officer surgery, Russels Hall Hospital, Birmingham.

- 1995-present : AGIKO Radiology: Resident, University Hospital Maastricht. Research assistent, University Hospital Maastricht and University of Maastricht.

\section{Published articles}

Ho K.Y.J.A.M., de Haan M.W., Oei T.K., Koster D., Kessels A.G.H., Janevski B.K., Kitslaar P.J.E.H.M., van Engelshoven J.M.A..

MR angiography of the iliac and upper femoral arteries using four different inflow techniques.

AJR Am J Roentgenol. 1997 Jul;169(1):45-53.

Ho K.Y.J.A.M., de Haan M.W., Kessels A.G.H., Kitslaar P.J.E.H.M., van Engelshoven J.M.A..

Peripheral vascular tree stenoses: detection with subtracted and nonsubtracted MR angiography.

Radiology. 1998 Mar;206(3):673-81.

Ho K.Y.J.A.M., Leiner T., de Haan M.W., Kessels A.G.H., Kitslaar P.J.E.H.M., van Engelshoven J.M.A..

Peripheral vascular tree stenoses: evaluation with moving-bed

infusion-tracking MR angiography.

Radiology. 1998 Mar;206(3):683-92.

Ho K.Y.J.A.M., Leiner T., van Engelshoven J.M.A..

MR angiography of run-off vessels.

Eur Radiol. 1999 Aug 23;9(7):1285-1289.

Leiner T., Ho K.Y.J.A.M., Thelissen G.R.P., Nelemans P.J., de Haan M.W., van Engelshoven J.M.A..

Contrastversterkte kernspinresonantieangiografie.

Ned Tijdschr Geneeskd 199922 mei;143(21). 


\section{In press}

Ho K.Y.J.A.M., Leiner T., van Engelshoven J.M.A..

Peripheral MR angiography.

European Radiology.

\section{$\underline{\text { Submitted }}$}

Ho K.Y.J.A.M., Leiner T., de Haan M.W., Kessels A.G.H., Kouwenhoven M., van Engelshoven J.M.A..

Analysis of imaged interpretability in contrast-enhanced peripheral MR angiography using the MoBI-track technique.

Ho K.Y.J.A.M., Leiner T., van Engelshoven J.M.A..

Peripheral vascular tree stenoses: Evaluation with bloodpool-enhanced and Gd-DTPA-enhanced MR angiography.

\section{Various:}

Abstract awarded with the Dutch Radiologists price 1997:

Gadolinium optimized tracking (GOT) technique: A new MRA technique for imaging the peripheral vasculature.

Ho K.Y.J.A.M., Leiner T., de Haan M.W., Kessels A.G.H., Kitslaar P.J. E.H.M., van Engelshoven J.M.A..

Abstract nominated for the Dutch Radiologists price 1998:

Prospectieve vergelijking van de diagnostische waarde van MoBI-track MR angiografie met duplex ultrageluidsonderzoek in het diagnostisch traject van patienten met claudicatio intermittens.

Leiner T., Ho K.Y.J.A.M., de Haan M.W., Kitslaar P.J.E.H.M., van Engelshoven J.M.A..

\section{$\underline{\text { Presentations/posters }}$}

Erasmus course on MRA, Cardiovascular and Interventional Applications of MRI

- Eighth meeting, Leiden, October 1998.

- Peripheral MR angiography.

- Nineth meeting, Leiden, November 1999.

- Technical details to contrast-enhanced MR angiography.

European Congres of Radiology

- 2000, Vienna, Austria, March 2000.

- Refresher course: MRI - From basic knowledge to advanced strategies: Run-off vessels. 
International Society for Magnetic Resonance in Medicine

- Fourth annual meeting. New York, United States, April 1996.

- Three dimensional high resolution contrast-enhanced subtraction MR angiography of the iliac arteries compared with X-ray angiography.

- Fifth annual meeting. Vancouver, Canada, April 1997.

- Gadolinium Optimized Tracking Technique: A New MRA Technique for Imaging the Peripheral Vascular Tree from Aorta to the Foot Using One Bolus of Gadolinium.

- Sixth annual meeting. Sydney, Australia, May 1998.

- Preliminary Results of an Operator Independent Peripheral MR Angiographic Outflow Study (MoBI-track) in 4 minutes (poster).

- Satelite refresher course: Peripheral MR angiography.

- Seventh annual meeting. Philadelphia, United States, May 1999.

- Keynote talk: Peripheral MR angiography.

- Image Interpretability of Peripheral MRA Using the MoBI-track technique.

- Comparison Between Bloodpool-Enhanced and Gadolinium-Chelateenhanced MR Angiography for Imaging the Peripheral Arteries (poster).

- Eighth annual meeting, Denver, United States, April 2000.

- Refresher course: Peripheral MR angiography.

International Workshop on Magnetic Resonance Angiography

- Eighth meeting, Rome, Italy, October 1996.

- Peripheral MRA.

- Nineth meeting, Valencia, Spain, October 1997.

- MoBI-track MR angiography; clinical applications.

- Tenth meeting, Utah, United States, October 1998.

- New applications in peripheral MR angiography.

- Eleventh meeting, Lund, Sweden, September 1999.

- Bloodpool-enhanced MR angiography.

Scientific Assembly and Annual Meeting of the RSNA

- 82nd meeting, Chicago, United States, December 1996.

- Cardiac-triggered time-of-flight MR angiography and nonsubtracted and subtracted gadolinium-enhanced MR angiography of the iliac arteries Gompared with X-ray.

- 83rd meeting, Chicago, United States, December 1997.

- Moving Bed Infusion tracking technique: A new MR angiographic technique for imaging the peripheral arteries.

- 85th meeting, Chicago, United States, December 1999.

- Peripheral vascular tree stenoses: Evaluation with bloodpool-enhanced MRA. 


\section{Dankwoord}




\section{Dankwoord}

Omdat het nederlands je makkelijker af gaat is deze regel voor jou ma.

Professor van Engelshoven:

Ik ben eigengereid, slordig, brutaal, en onstuimig. Het is de meester die van zo'n pupil een persoon maakt.

Professor Kitslaar:

Uw inzicht, inzet en bereidheid om buiten uw vakgebied mee te werken aan dit onderzoek was van onschatbare waarde.

Tim Leiner:

Professor Leiner: Tummi!

Fons Kessels:

Wat ik ook deed jij wist het statistisch significant te maken.

Vrijwilligers:

Mijn dank aan mijn vrijwilligers is rechtevenredig met het door hun geconsumeerde volume aan gadolinium:

$\begin{array}{lll}\text { Tim Leiner } & 163 & \text { liter } \\ \text { Dennis Laurens } & 159 & \text { liter } \\ \text { Michiel de Haan } & 3.6 & \text { liter } \\ \text { Etiënne Lemaire } & 316 & \mathrm{~mL} \\ \text { Marc Geerlings } & 15 & \mathrm{~mL} \\ \text { Koo de Priester } & 5 & \mathrm{~mL} \\ \text { Regina Beets } & \text { noppes } & \end{array}$


Jut: I've GOT it 

